\title{
Model Checking Reconfigurable Processor Configurations for Safety Properties ${ }^{\star}$
}

\author{
John Cochran, Deepak Kapur, and Darko Stefanović \\ University of New Mexico, Albuquerque NM 87131, USA, \\ cochran@es.unm. edu, \\ WWW home page: http://www.cs.unm.edu/ slugboy/index.html
}

\begin{abstract}
Reconfigurable processors pose unique problems for program safety because of their use of computational approaches that are difficult to integrate into traditional program analyses. The combination of proof-carrying code for verification of standard processor machine code and model-checking for array configurations is explored. This combination extends proof-carrying code to provide a context for model checking, but uses standard model checking technology. This approach is shown to be useful in verifying safety properties including the synchronization of memory access by the reconfigurable array and memory access bounds checking.
\end{abstract}

\section{Introduction}

Reconfi gurable computing is a rapidly evolving technology that has great potential for improved processing effi ciency for a variety of important computational tasks. This improvement, however, comes at the expense of increased risk of problems from faulty or malicious programming. Although methods of mitigating this risk have not been explored deeply in the literature, this will be necessary for the success of the technology.

We are exploring model checking combined with proof-carrying code as a potential method of ensuring safety of reconfi gurable processor programs. This exploration will be in the context of the Garp reconfi gurable processor. We show that signifi cant safety properties can be efficiently and automatically verifi ed by model checking. However, we also report that model-checking some other properties of interest is not effi cient, and we consider possible directions for addressing this shortcoming.

\section{Safety for Reconfi gurable Computing}

There are several reasons that cause reconfi gurable processors to pose an increased safety risk: reconfi gurable processors entail greater consequences of unsafe operation because reconfi gurable fabrics can be destroyed by some improper uses; reconfi gurable processors have more complex functionality; the large variability of computations and fi ne-grained concurrency make programming reconfi gurable fabrics more diffi cult; reconfi gurable confi gurations are also less accessible and less modifi able; and fi nally, the

\footnotetext{
* Partially supported by the NSF Grants nos. CCR-9996150 and ITR-CCR-0113611.
} 
computation on a reconfi gurable fabric cannot in general be augmented by instrumenting code because of the lack of space and tight timing issues.

Safety properties of programs are assertions that some bad event never happens during a computation. In contrast, correctness or liveness properties state that a computation produces correct results or that some event eventually happens during a computation. What the bad events are will be left to a safety policy which will depend on the context of program usage. Safety properties are generally easier to verify than correctness or liveness properties, which makes them a better candidate for formal methods.

Safety properties for reconfi gurable processors can be decomposed into properties of the standard part of the processor and properties of the reconfi gurable array. This decomposition assumes that the two sections of the processor are relatively independent. This is true of the Garp and similar processors but not true of reconfi gurable processors that implement specialized machine instructions in the reconfi gurable array.

Each of these types of safety property is further subdivided into generic properties that must be true for all instances (programs and array confi gurations) and context dependent properties that must be true of individual instances and that are derived from a safety policy. Generic safety properties include such properties as not running code outside of the program code segment and only calling functions at code locations where functions reside (for the standard part of the processor), and avoiding memory bus conflicts and undefi ned confi gurations (for the reconfi gurable array).

Unfortunately, there is no obvious method of verifying both the standard and reconfi gurable parts of the program in a single framework. Proof-carrying code works well for sequential instructions that can be symbolically evaluated to produce a verifi cation condition, but does not address the concurrent, instructionless computation performed by the reconfi gurable array. On the other hand, model checking works well for fi nite state, concurrent systems such as the reconfi gurable array, but has problems dealing with infi nite state systems such as standard program code.

The novel approach explored here uses model checking to verify safety properties of the reconfi gurable array, and proof-carrying code to verify safety properties of the standard machine code. In addition, proof-carrying code provides a context for model checking the reconfi gurable array by providing preconditions, postconditions, a memory partition to prevent memory conflicts between the standard processor executions and array executions while the array is running, and local safety properties. This is achieved by extended Proof-carrying code's context mechanism for function calls to reconfi gurable array executions.

The context is needed for model checking the array confi gurations because simply checking the confi guration for safety properties without any external context would restrict the class of such properties greatly. For example, any property that relies on the correct initialization of array registers cannot be verifi ed unless there is some external assurance that the registers are in fact correctly initialized.

\section{Proof-Carrying Code}

Proof-carrying code (PCC) [NL97,Nec97,Nec98] is a method of ensuring the safety of untrusted machine code. It relies on the code producer to produce a verifi cation of safety and transmit evidence of the verifi cation to the code consumer. 


\subsection{Overview}

The first component of PCC is a safety policy, which is specifi ed by the developer of the system and which can vary widely over a large space of possible policies. There is no notion of a universally acceptable safety policy for all systems in PCC, although there is a common infrastructure that is part of the fi nal safety policy specifi cation. Each system must defi ne a safety policy (or a set of such policies) using the common infrastructure and advertise it to developers of programs for the system.

The program developer must provide a proof of compliance with the advertised safety policy. This proof, the second component of PCC, must be in a specifi c format to allow automated checking. This also means that the proof must refer directly to the machine code and not an abstracted version of the program. How the proof is produced does not matter for the PCC system.

The third component of PCC is a proof checker for the client. When the client receives code from an untrusted source it examines the code along with the attached proof to make sure that the code obeys the safety policy. This checking must be automated, fast, and highly reliable. It must be the case that if the proof checks then it is safe to run the code, according to the advertised policy. Thus it does not matter if the code or the proof is tampered with or garbled. If the proof still checks, the code will be safe to run even if it does not necessarily perform its expected function.

The main advantage of PCC is that the hard work-compiling the code and producing the proof-is in the hands of the code producer, while only the easy work, checking the proof and running the program, is required of the code consumer. This asymmetry allows the hard work to be done once and used many times. This is opposed to analyzing the code every time a new client uses it. This alternative is not only wasteful of processor time for the client, but much harder than having the producer give a proof, as the client has less information about the code than the producer-no source code, no formal or informal specifi cations, etc.

\subsection{Extensions}

We have extended the PCC system for a reconfi gurable array. The semantics of safe program execution and the symbolic evaluator that produces the safety predicate have been extended to treat array executions as a type of function call. The extension includes new instructions for accessing array registers, loading confi gurations, and starting array execution. The semantics and symbolic execution of old instructions are updated to take into account the reconfi gurable array execution state. These extensions are necessary to provide the correct semantics of instructions reading array registers. If these instructions are called during array execution, the value read is indeterminate, otherwise it is available to the symbolic evaluator. The extensions are documented in [Coc02].

A big difference between regular function calls and reconfi gurable array executions is that the computation calling a function waits for it to return while the computation initiating an array execution may continue a concurrent computation. We model this in the safety semantics by the use of two variants of Hilbert's $\varepsilon$-calculus [Lei69].

One version models values in the reconfi gurable array that are inaccessible by the standard part of the program with the $\varepsilon$ constructor. This constructor follows the rules:

$$
\exists v \cdot F v \supset F\left(\varepsilon_{\alpha} v \cdot F v\right)
$$




$$
\forall v(F v \equiv G v) \supset \varepsilon_{\alpha} v \cdot F v=\varepsilon_{\alpha} v \cdot G v
$$

This states that for any locations $\alpha$, if it can contain a value for which $F$ holds, then $F$ holds of the value $\varepsilon_{\alpha} v . F v$, and any such constructed value is unique. $\varepsilon$-terms can occur in the symbolic evaluation of the program, but not the fi nal property to be proved.

The other version models values computed by the reconfi gurable array that are nondeterministic to the standard part of the processor. This version is called $\eta$-calculus ${ }^{1}$, and follows the rule:

$$
\exists v \cdot F v \supset F\left(\eta_{\alpha} v \cdot F v\right)
$$

This states that for any locations $\alpha$, if it can contain a value for which $F$ holds, then $F$ holds of the value $\eta_{\alpha} v . F v$. There is no guarantee of uniqueness for $\eta$-terms. $\eta$-terms only occur in the semantics and are used to prove soundness.

In addition to the property to prove for the standard section of the program, the extension provides preconditions and postconditions for array execution, partitions the memory between the standard processor and reconfi gurable array, and provides a context for the safety policy that allows a concrete realization of safety properties. The preconditions state what values can be expected to be placed into the reconfi gurable array registers at the start of array execution, as well as the number of cycles the array will run. The postconditions state what values can be expected to be left in the reconfi gurable array registers following array execution. The memory partition simply states which addresses the reconfi gurable array can access without interference from the rest of the processor. The safety properties to be checked are evaluations of the safety policy in the state reached by the symbolic evaluator at the time of array execution.

\section{Model Checking}

Model checking can be easily summarized as a simple problem dealing with machines and temporal propositions [CGP99]. Assume that there is some fi nite state machine $M=\left\{S, S^{\prime}, T, L\right\}$, where $S$ is the set of states, $S^{\prime} \subseteq S$ is the set of initial states, $T \subseteq S \times S$ is a total transition relation, and $L: S \rightarrow 2^{A}$ is an injective function from states to sets of state variables $A$ labelling those variables that are true in the state. The model-checking problem is then to determine, for any temporal formula $f$, those states $S_{t} \subseteq S$ for which $f$ is true.

Model checking is used to verify safety properties of the reconfi gurable array. Model checking is a formal verifi cation method that automatically examines fi nite models of concurrent systems to determine the properties of the models. These verifi cation properties can be expressed in any one of a variety of logics but propositional temporal logics give a good balance between expressibility and effi ciency of checking. In this work we use RTCTL [Cam96], a branching-time propositional temporal logic [Pnu77]. RTCTL uses bounded temporal quantifi ers to implement bounded model checking.

RTCTL formulas express ways in which propositions can be true over a branching model of time. For example,

$$
\text { ABG } 0 . .1023(x<y+2048)
$$

\footnotetext{
${ }^{1} \eta$-calculus is called $\kappa$-calculus in [Coc02], but was later found to be equivalent to $\eta$-calculus as discussed in [Lei69].
} 
states that on all paths from the current time, at all times from now until 1023 time steps later, $x$ is less than $y$ plus 2048, and

$$
\mathbf{A F}(x=0)
$$

states that on all paths, at some time from now on, $x$ is equal to 0 . The fi rst of these examples is a safety property over a bounded time period, while the second is a liveness property over all time. Safety properties can be easily identifi ed in RTCTL because they have an outer temporal quantifi er of $\mathbf{A G}$ for infi nite temporal progressions or $\mathbf{A B G}$ for fi nite temporal progressions if the bound is large enough to cover the entirety of the temporal progression.

Model checking does not have to be extended for our purposes. Off-the-shelf model checkers provide rich enough description languages to describe the system to be modeled, and specifi cation languages to specify the properties to be checked. In particular, we have used the NuSMV system [CR98] for the verifi cation of array confi gurations. NuSMV uses the language SMV to describe models. This language is similar to hardware description languages or process algebras. As specifi cation language, NuSMV supports RTCTL. For RTCTL model checking, NuSMV uses ordered binary decision diagrams (OBDDs) as a symbolic representation of the model and specification [Bry92]. This use of symbolic representations greatly improves the effi ciency of model-checking for array confi gurations.

\subsection{Building Models for Confi gurations}

Before model checking a confi guration, it is necessary to build a model for it. The usual practice in model checking systems is to build a model in a description language before implementing the system. In our case, the code consumer does not have any information about confi gurations except their bit level encoding and the machine code where they occur. Thus we build the model by translating the bit level encoding to the SMV language. This uses only the bit level encoding; any information from the surrounding machine code that is needed to verify safety must be used in the safety properties for the confi guration. Details can be found in [Coc02].

\section{The Garp Processor}

The reconfi gurable processor which we use as our example is the Garp processor designed at Berkeley [Hau00,HW97,Hau97]. This processor has not been physically implemented but it has been thoroughly specifi ed and documented, which is critically important for proving safety properties. The documentation and the ability of the reconfi gurable array to independently access memory were the deciding factors when we chose Garp. The basic feature of the Garp design that will be considered is the reconfi gurable logic array.

The Garp logic array is an array of blocks arranged into 24 columns. The leftmost block in each row is a control block and all the others are logic blocks. There is an

unspecifi ed number of rows in the array (but there cannot be more than 32 because of the constraints of the confi guration fi le format). 
Each logic block has two 2-bit clocked registers. There are four 2-bit inputs to the logic blocks that can come from any wire pair accessible to the block, from latched registers, or from the binary constants 00 or 10 . The outputs can be the registers, the block function output, or one of the block inputs.

The table mode takes the four 2-bit main inputs and outputs two bits depending on a lookup table. The split table mode is similar but has two separate tables for the fi rst and second bits. The select mode implements a multiplexer which uses one input as control and selects between the other inputs. The inputs can be shifted to the left or inverted. The partial select mode is identical to select mode with the exception of which inputs are available. The carry chain mode and the triple add mode are more complicated modes that can be used to implement functions requiring fast carry chains or arithmetic on three inputs.

Other functions of the logic array are initiated by the control blocks. Of primary interest, memory loads and stores can be initiated by the control blocks. This is independent of the standard processor, but accesses go through the same memory hierarchy as for the standard processor. There is only one memory address bus, so only one access can be initiated at a time. A control block can load or store the registers in its row over one of four memory data busses. More detailed information on the array features can be obtained from the Garp Architecture Manual [Hau97].

\section{Safety Problems with Garp}

A safety problem with the Garp reconfi gurable array is that only one memory access may be initiated per cycle although more than one control block could be triggered to access memory depending on the state of the array. Thus it is possible for a perfectly legal confi guration to attempt to perform an illegal action. A similar problem is that two memory accesses initiated at different times could illegally schedule the transfer of data for the same cycle. These actions could lead to unexpected behavior because there is no specifi cation of what should happen in these circumstances. Because they cannot be checked syntactically, these control problems must be checked in some other manner. These problems are addressed by generic safety properties as discussed in Section 7.

Accessing and writing of data at addresses forbidden by a safety policy is another safety problem. Such accesses could lead to the unwanted access to private information, buffer overruns [CWP $\left.{ }^{+} 00\right]$, stack smashing [One96], and similar problems. These can occur in perfectly legal confi gurations and programs but are very troublesome. Common solutions to these problems in standard processors, boundary checks by the language, programmer, or analysis tool, do not work for the reconfi gurable array. There is no suitable method to make the hardware handle these problems either. A method of preventing them in untrusted code is critical to ensure safety, therefore our work focuses on these problems. These problems are addressed by context dependent safety properties as discussed in Section 7.

Another class of safety problems is not addressed here. These include illegal block confi gurations in the encoding, multiply driven wires, and clock skew issues. Fortunately this type of safety problem has been investigated by Hauser and can be detected by hardware validation of confi gurations before they are loaded [Hau00]. Hauser also 
mentions dynamically checking for illegal actions by legal confi gurations in the hardware, but here we show that it is possible to check for these problems statically.

\section{Properties to be Model Checked}

Once a confi guration is translated into SMV it can be checked for many properties. These properties fall into three classes:

- Generic memory control properties.

- Context dependent memory access properties.

- Context dependent postcondition properties.

Each of these properties can rely on the preconditions for the confi guration, so the specifi cations are implications with the preconditions as an antecedent and the safety specifi cations as the consequent. Typically, the preconditions reflect array register and counter initialization, while the postcondition reflects assumptions that can be made about values in the array registers following execution.

The generic properties which will be checked for all confi gurations include:

- At most one memory access is initialized per clock cycle.

- At most one memory access is scheduled to use the bus for each cycle.

- There is a memory item ready to read when a row reads one.

- There is a row initiating a memory write when a row transfers to memory.

These properties all deal with synchronizing memory accesses so that they are defi ned by the semantics of Garp's reconfi gurable array. If any of these properties is false, then there can be undefi ned behavior from the array. From a list of which control blocks can initiate memory accesses, it is possible to deduce these specifi cations.

The context dependent safety properties for a particular confi guration include:

- All memory accesses respect the memory partition.

- All memory accesses respect the memory access safety properties.

- The postcondition is true after array execution.

These properties all rely on information from the safety policy and the symbolic evaluation of the program at the point where the array confi guration is executed. Because the array uses standard binary representations for addresses, the confi guration cannot be too devious in its memory accesses. The preconditions and postconditions must be in a standard representation because they are used in the verifi cation of the standard code as well as the array. This allows a coherent translation of the properties expressed with standard program types such as integers and characters as primitives, and the model checking version which is expressed with bits as primitives.

Either of these types of properties can also use information about the initial count for the array execution. This count gives the number of cycles that the array executes if it does not halt itself fi rst. The count is used in the bounds for temporal quantifi ers such as $0 . .5$ in Equation 4. 


\section{Performance of NuSMV on Translated Input Files}

The performance of the model checker, NuSMV 2.0 running on an AMD Athlon at $1900 \mathrm{MHz}$ with $1024 \mathrm{MB}$ of memory under Debian Gnu/Linux 2.2, is the main factor to be explored for performance. Four example confi gurations were checked for six properties, the first of which is a generic property and the rest of which are context dependent properties:

- Memory control safety (MC).

- Memory alignment (MA).

- Lower bounds for memory reads (LBR).

- Upper bounds for memory reads (UBR).

- Lower bounds for memory writes (LBW).

- Upper bounds for memory writes (UBW).

The four confi gurations include three that perform the same function, but have different control paths and preconditions. The application is to read 200 word-sized pixels from an array, lighten each color component, and write the results back to the array. This application was chosen to reflect a common but problematic use of the reconfi gurable array, reading a writing memory with little interaction with the standard processor.

The fi rst confi guration (IM1) has the precondition that the register in the fifth row of the reconfi gurable array is loaded with a value equal to the value loaded in the fi rst row of the reconfi gurable array minus 14 . This is because the fi rst row reads the pixel array, the fifth row writes the result back, each of them is incremented by 2 on each cycle, and it takes seven cycles for the computation. The second confi guration (IM2) has the precondition that the values loaded into the fi rst and fi fth row registers are equal. This is because the fifth row does not start incrementing until it is signaled on cycle 7 by the control path. The third confi guration (IM3) does not have any corresponding precondition because it passes address from the fi rst row to the fi fth alongside the computation so that the value in the fifth row is always correct.

The fourth confi guration (HASH) is a hash table. It is included to check the effect of computed addresses on the model checking. It simply reads values from an array, computes a 10-bit offset by repeated shifts and exclusive ors, and writes the value to an address plus the offset.

Each of the the confi gurations has preconditions to ensure that the control path is correctly initialized, and that the access locations fi t into memory without any wraparound. The correct initialization preconditions are simply assertions that certain registers have certain values, while the memory fit preconditions are disjunctions of assertions that certain register locations have the value 0 . All of the confi gurations have the trivial postcondition true because they do not leave any values in the array registers for later use. They only affect memory.

The results are presented in Table 1 for unbounded properties, and Table 2 for bounded properties. The memory write properties for IM1, IM3, and HASH do not appear in Table 2 because they did not fi nish model checking in under four days. Further information on the confi gurations and properties is available in the appendices. 
The reasons for the poor performance on the write boundary properties (LBW, UBW) are varied. For IM1, the precondition requires a very long bit level specifi cation that makes even trivial specifi cations practically uncheckable. The other properties did not rely on this precondition, so they could be checked without it. IM3 and HASH both have a write address that is dependent on several rows of registers and cycles. This seems to be more than the model checker can handle effi ciently. Even for IM2 it took a signifi cantly longer time to check the write boundary properties as they depend on more of the array than the rest of the properties. IM3 and HASH took longer to check on the other properties because they are about two and three times as large as the other confi gurations. Thus the longer times are not unexpected.

This investigation of performance is far from complete, but it shows that common safety properties are effi ciently checkable for certain types of confi gurations. Unfortunately, there is not a wider base of applications that could be used to produce realistic data, since Garp is not implemented.

\begin{tabular}{|l|r|r|r|r|}
\hline & \multicolumn{4}{|c|}{ Configuration } \\
\hline Property & IM1 & IM2 & IM3 & HASH \\
\hline MC & 6.710 & 6.140 & 6.810 & 17.400 \\
MA & 6.740 & 13.690 & 14.140 & 33.670 \\
\hline
\end{tabular}

Table 1. Results of Model Checking Example Configurations for Unbounded Properties. Model checking times are given in seconds.

\begin{tabular}{|l|r|r|r|r|r|r|r|}
\hline & \multicolumn{7}{|c|}{ Time Step Bound } \\
\hline Configuration / Property & 400 & 1000 & 1024 & 4000 & 4096 & 16000 & 16384 \\
\hline IM1 LBR & 15.23 & 16.84 & 16.92 & 24.10 & 24.42 & 54.00 & 54.76 \\
IM1 UBR & 16.97 & 20.66 & 20.26 & 38.78 & 36.95 & 106.72 & 98.70 \\
IM2 LBR & 15.13 & 16.55 & 16.65 & 23.20 & 23.12 & 49.38 & 50.04 \\
IM2 UBR & 16.59 & 19.98 & 19.99 & 36.57 & 36.57 & 102.92 & 94.32 \\
IM2 LBW & 64.00 & 76.11 & 70.52 & 141.71 & 88.37 & 161.54 & 414.43 \\
IM2 UBW & 69.64 & 87.61 & 76.30 & 109.13 & 197.60 & 240.14 & 239.10 \\
IM3 LBR & 16.12 & 18.63 & 18.91 & 30.70 & 31.22 & 80.50 & 81.77 \\
IM3 UBR & 18.00 & 23.73 & 23.22 & 48.70 & 47.40 & 147.10 & 142.53 \\
HASH LBR & 27.27 & 29.30 & 29.20 & 38.84 & 39.29 & 78.56 & 79.93 \\
HASH UBR & 28.42 & 32.99 & 32.65 & 58.93 & 57.52 & 168.92 & 169.41 \\
\hline
\end{tabular}

Table 2. Results of Model Checking Example Configurations for Bounded Properties. Model checking times are given in seconds.

\section{Conclusions and Further Work}

The main result of this work is that model checking reconfi gurable processor confi gurations is a viable verifi cation method for important safety properties. Although some properties have been found to be too complex for effi cient checking, there may be methods to mitigate this in many cases. As the examples show, confi gurations having equivalent computations with different control strategies can have very different behavior when model checked. This could be taken into account in a compiler designed to produce effi ciently checkable confi gurations. 
Unfortunately, some properties seem to be uncheckable for practical purposes. There is no way to implement the hash table application without computing the write address, but even trivial computed address such as in IM3 do not check in a reasonable time.

Integrating model checking into a synoptic system of program verifi cation in order to solve these problems is being explored. In particular, replacing proof-carrying code based on fi rst-order logic with proof-carrying code based on temporal logic [BL02] is expected to provide a greater range of safety properties that can be checked. This may allow model checking of easy-to-check properties, which are then integrated into the proof of safety attached to the program as a whole.

In the hash table example, the model could be decomposed into small pieces involving only a single cycle. The pieces could then be model checked effi ciently and the checked properties combined to yield a proof of safety. This would be possible because the proof-carrying code infrastructure would be able to check the correctness of temporal logic proofs.

\section{References}

[BL02] A. Bernard and P. Lee. Temporal logic for proof-carrying code. Technical Report CMU-CS-02-130, School of Computer Science, Carniege Mellon University, Pittsburgh, PA, 2002.

[Bry92] R.E. Bryant. Symbolic Boolean manipulation with ordered binary-decision diagrams. ACM Computing Surveys, 24(3):293-318, 1992.

[Cam96] S.V. Campos. A Quantitative Approach to the Formal Verification of Real-Time Systems. PhD thesis, Carnegie Mellon University, 1996.

[CGP99] E.M. Clarke, O. Grumberg, and D.A. Peled. Model Checking. MIT Press, Cambridge, MA, 1999.

[Coc02] J. Cochran. Towards provably safe reconfigurable processor code: A model checking and proof-carrying code approach. Master's thesis, University of New Mexico, 2002. Available as Technical Report TR-CS-2002-36.

[CR98] A. Cimatti and M. Roveri. NuSMV 1.1 User Manual. ITC-IRST and CMU, 1998.

$\left[\mathrm{CWP}^{+} 00\right]$ C. Cowan, P. Wagle, C. Pu, S. Beattie, and J. Walpole. Buffer overfbws: Attacks and defenses for the vulnerability of the decade. In DARPA Information Survivability Conference and Exposition (DISCEX 2000), pages 119-129, January 2000.

[Hau97] J. Hauser. The Garp Architecture. University of California at Berkeley, Department of Electrical Engineering and Computer Science, Computer Science Division, Oct 1997.

[Hau00] J.R. Hauser. Augmenting a Microprocessor with Reconfigurable Hardware. PhD thesis, University of California, Berkeley, 2000.

[HW97] J.R. Hauser and J. Wawrzynek. Garp: A MIPS processor with a reconfigurable coprocessor. In Kenneth L. Pocek and Jeffrey Arnold, editors, IEEE Symposium on FPGAs for Custom Computing Machines, pages 12-21, Los Alamitos, CA, 1997. IEEE Computer Society Press.

[Lei69] A.C. Leisenring. Mathematical Logic and Hilbert's $\varepsilon$-symbol. MacDonald and Co., London, 1969.

[Nec97] G.C. Necula. Proof-carrying code. In Proceedings of the 24th ACM SIGPLANSIGACT Symposium on Principles of Programming Langauges (POPL '97), pages 106-119, Paris, January 1997.

[Nec98] G.C. Necula. Compiling with Proofs. PhD thesis, Carnegie Mellon University, 1998. 
[NL97] G.C. Necula and P. Lee. Safe untrusted agents using proof-carrying code. In Mobile Agents and Security, pages 61-89, 1997.

[One96] Aleph One. Smashing the stack for fun and profit. Phrack, 7(49), 1996.

[Pnu77] A. Pnueli. The temporal logic of programs. In 18th IEEE Symposium on Foundation of Computer Science. IEEE Computer Society Press, 1977.

\section{A Example Garp Array Confi gurations}

These confi gurations are given as $\mathrm{C}$ language arrays from a program that produces the confi gurations as binary fi les. Unfortunately, there is no available means for describing Garp confi gurations and producing binary fi les that is easily readable. After the header which gives the size of the confi guration in rows, each pair of words is the confi guration data for one Garp block. Because the translator from binary confi gurations to SMV expects the low numbered blocks before the higher numbered blocks, the order of the confi guration data seems backwards compared to the usual visual representation of the Garp array. Thus the control block confi guration data is in the last pair of words but these are not out of order with respect to the block confi guration defi nition.

\section{A.1 IM1}

The image manipulation confi guration with the write address initialized to 14 less than the initial read address. The control path begins with an block which emits a 1 pulse every other cycle. This pulse is used in the fi rst row to initiate loads, 4 cycles later it signals the second row to transfer from memory, and another 3 cycles later it signals the sixth row to initiate a store and the fi fth row to transfer to memory. The fi rst and sixth rows increment the addresses they contain by 2 every cycle.

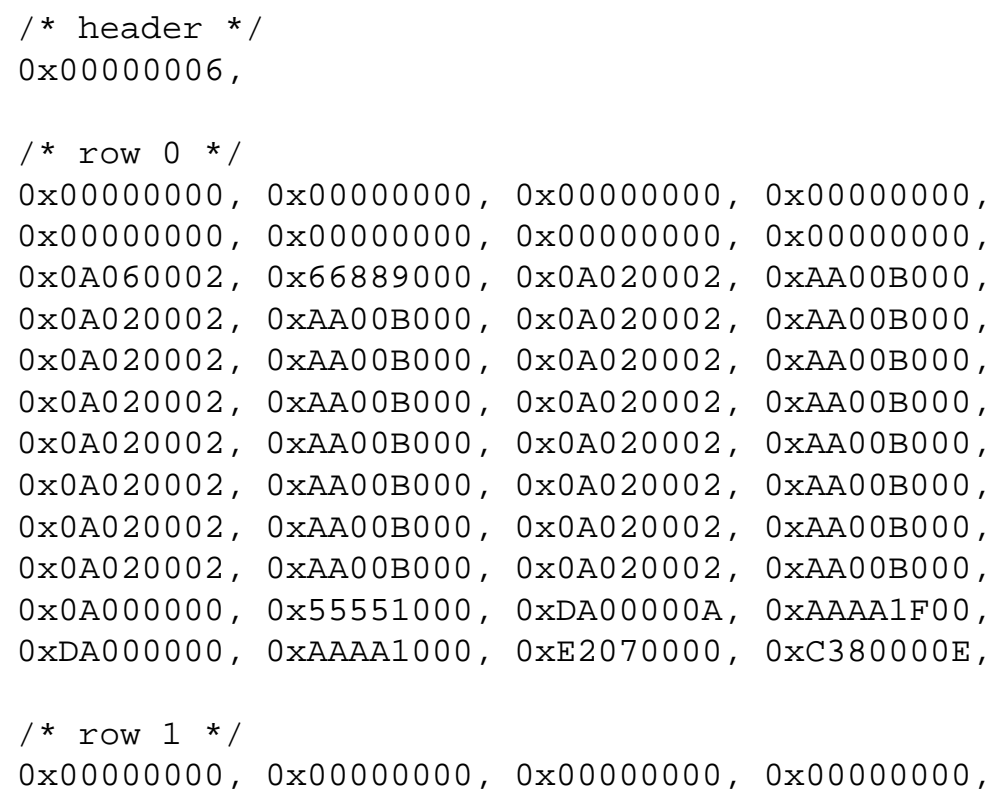




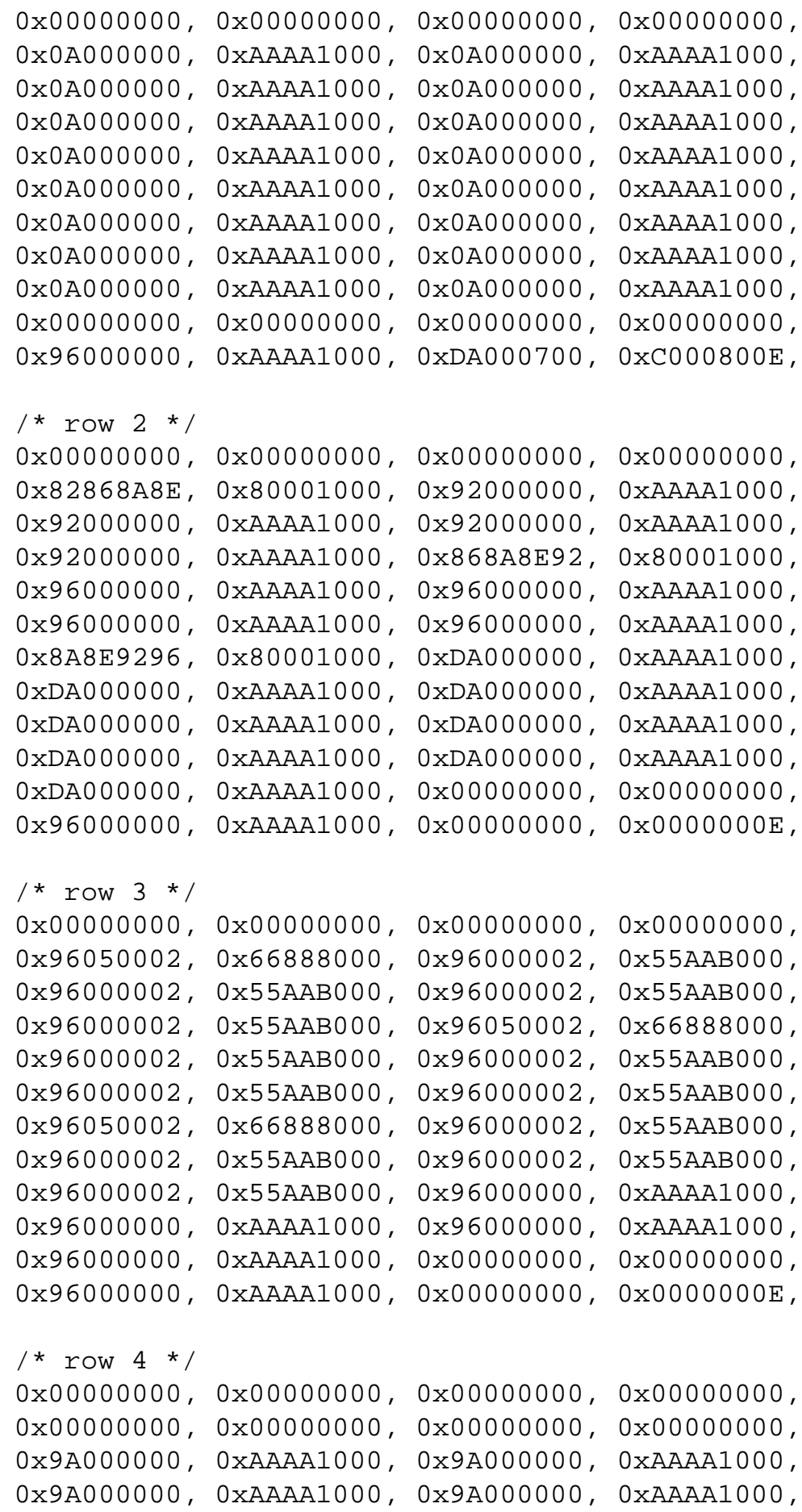




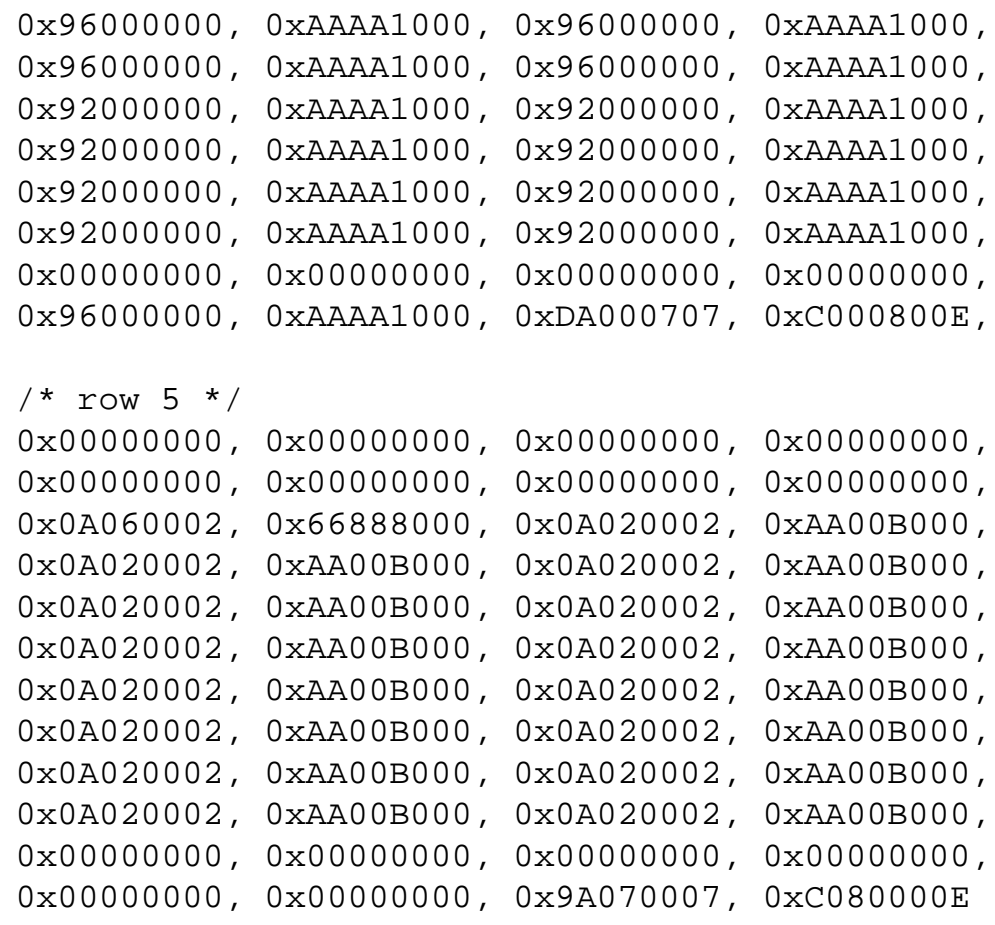

\section{A.2 IM2}

The image manipulation confi guration with the write address initialized to the same value as the initial read address. The control path is the same as IM1 but also signals the sixth row to begin incrementing after 7 cycles instead of having it begin incrementing on cycle 1 independently of the control path.

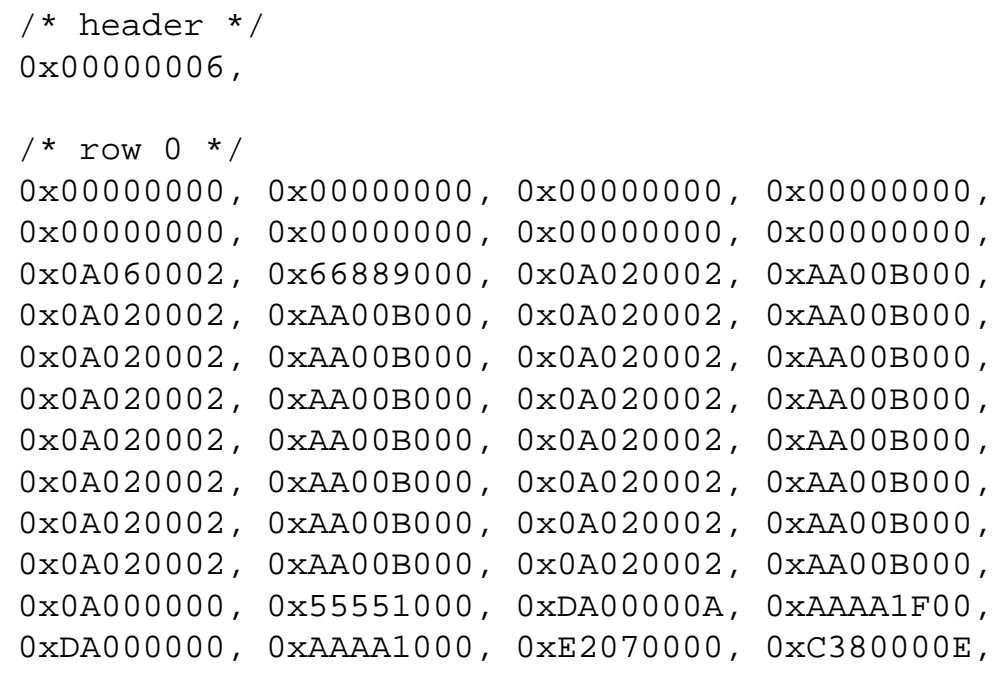




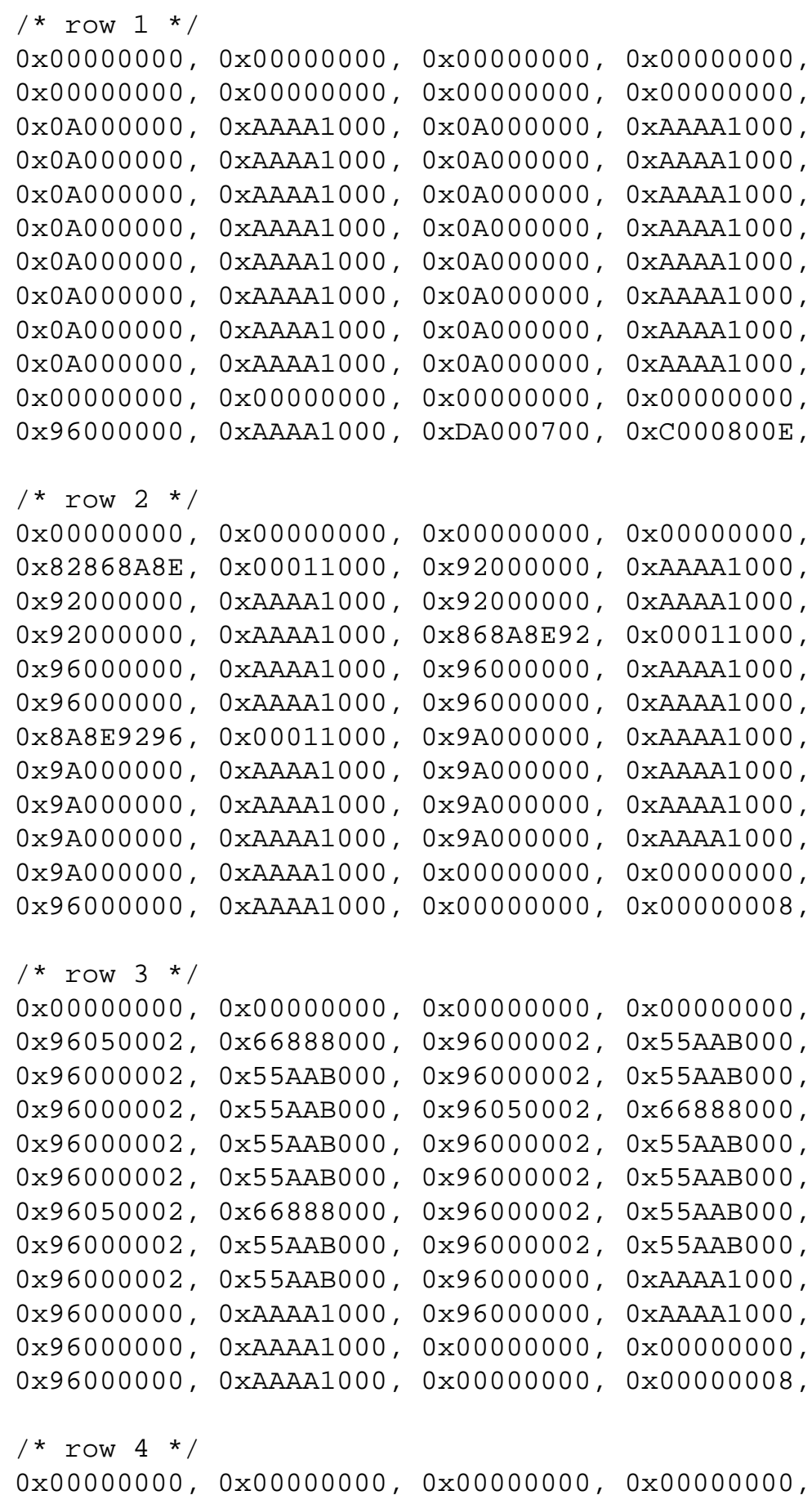




\begin{tabular}{|c|c|c|c|}
\hline & & & \\
\hline & AA1 & 0 , & \\
\hline 00000 & $0 \times A A A A 1000$ & 000000, & \\
\hline 6000000 & $0 \times A A A A 1000$, & 000000, & AAAAD \\
\hline 96000000 & $0 \times A A A A 1000$ & $0 \times 96000000$ & 0 \\
\hline 92000000 & $0 \times A A A A 1000$, & 00000, & \\
\hline 92000000 & $0 \times A A A A 1000$ & 000000, & \\
\hline 92000000 & $0 \times A z$ & 00, & \\
\hline 0 & $0 \times A$ & & \\
\hline 00, & & & \\
\hline 0 & $0 \times A z$ & & \\
\hline - & & & \\
\hline & 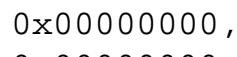 & & \\
\hline 800 & $0 \times 0$ & & \\
\hline 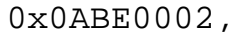 & $0 \times 66888$ & $0 \times 0$ & 0 \\
\hline 80 & OXAAOOBOOO, & $0 \times 0$ & $0 \times A$ \\
\hline 穴 & $0 \times A A 00 B$ & $0 \times 0$ & $0 x$ \\
\hline$\times 0 z$ & OXAA00B 000, & $0 \times 0$ & $0 \times A$ \\
\hline $\mathrm{x} 0 \mathrm{z}$ & $0 \times A A 00 B 0$ & $0 \times 0$ & $0 \times A A$ \\
\hline$x^{2}$ & OXAAOOBOC & $0 \times 0$ & $0 \times A A$ \\
\hline $\mathrm{xOF}$ & OXAAOOBOO & $0 \times 0 A 020002$ & $0 \times A A$ \\
\hline ( & OXAAOOBO & $0 \times 0$ & $\mathrm{BAA}$ \\
\hline & $0 \times 00000$ & $0 \times 0$ & $x 00$ \\
\hline & 3000000 & 位 & $\times C 0800$ \\
\hline
\end{tabular}

\section{A.3 IM3}

The image manipulation confi guration with the write address passed from the fi rst row to the sixth row via the D registers. The control path is the same as IM1.

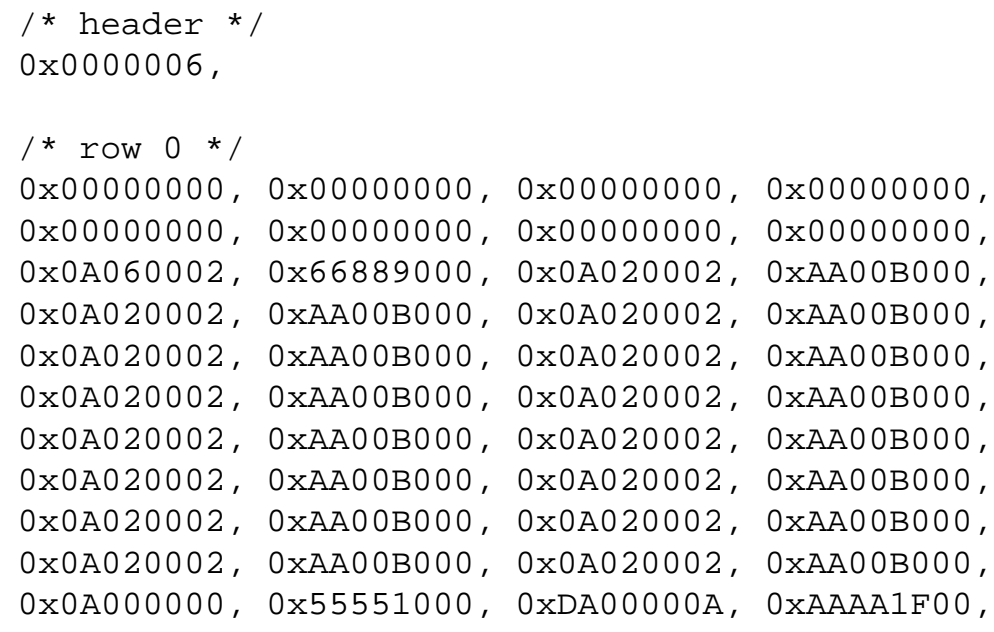




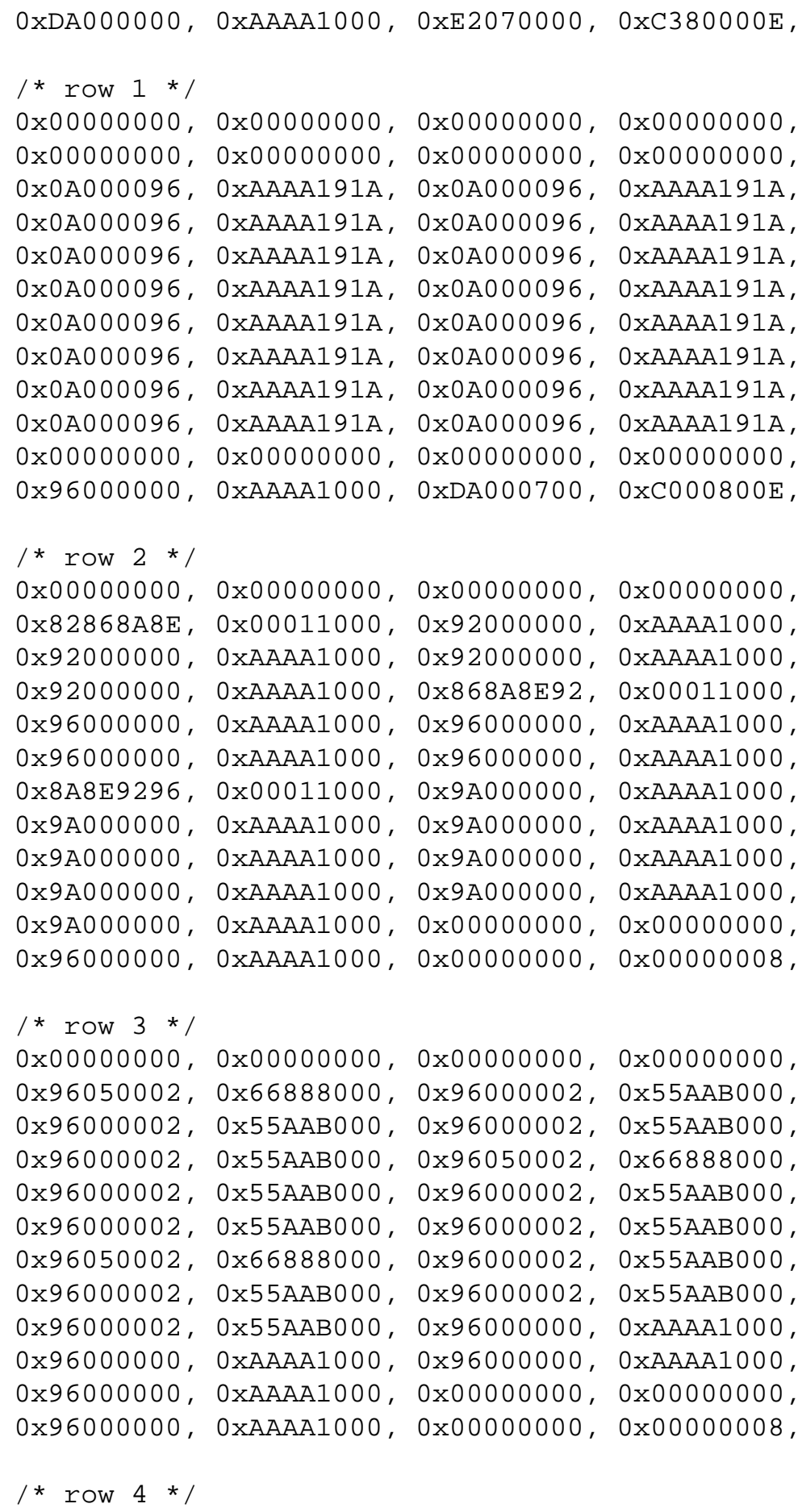




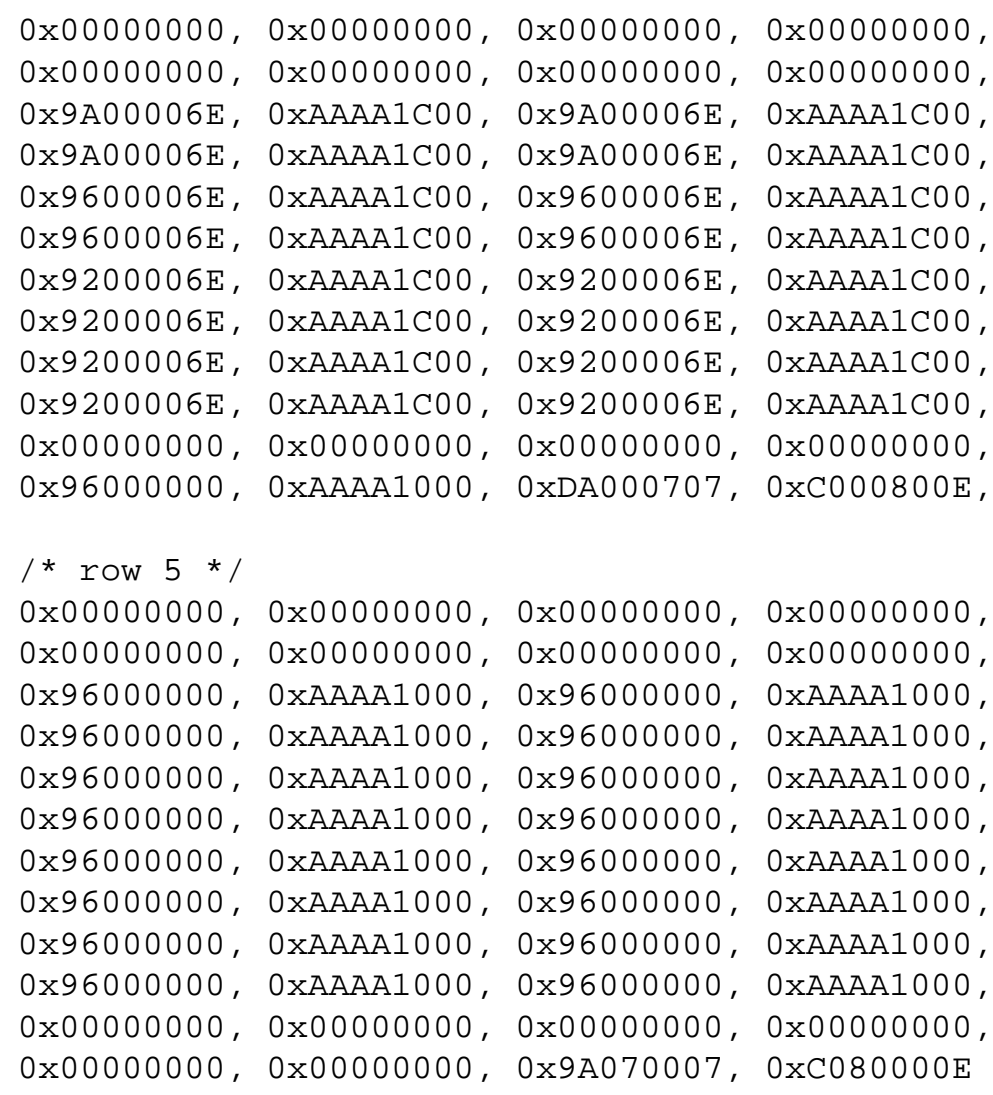

\section{A.4 HASH}

The hash table example. The control path is similar to that of IM1 but has a longer delay because the confi guration has more rows. The store address is independent from the control path because it is computed as an offset from the start of the hash table by a repeated process of shifting and exclusive oring bytes into the following byte. The most signifi cant bytes are zeroed as soon as possible and copied to subsequent rows during the computation.

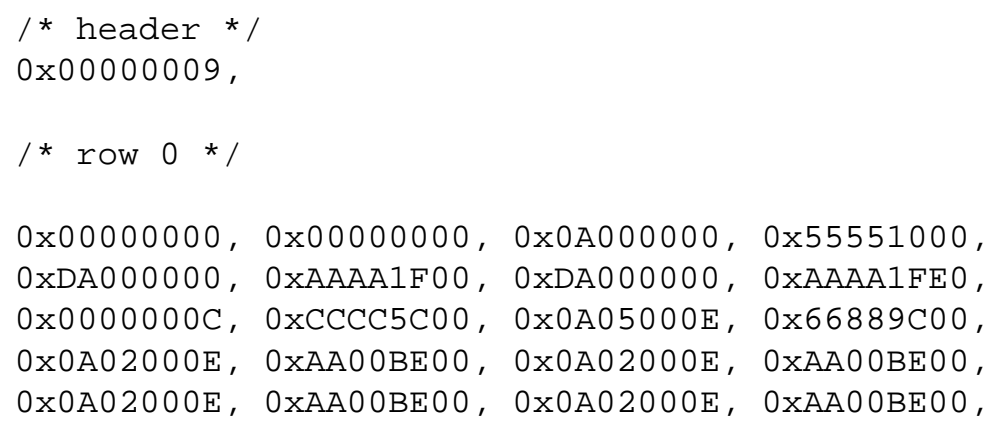




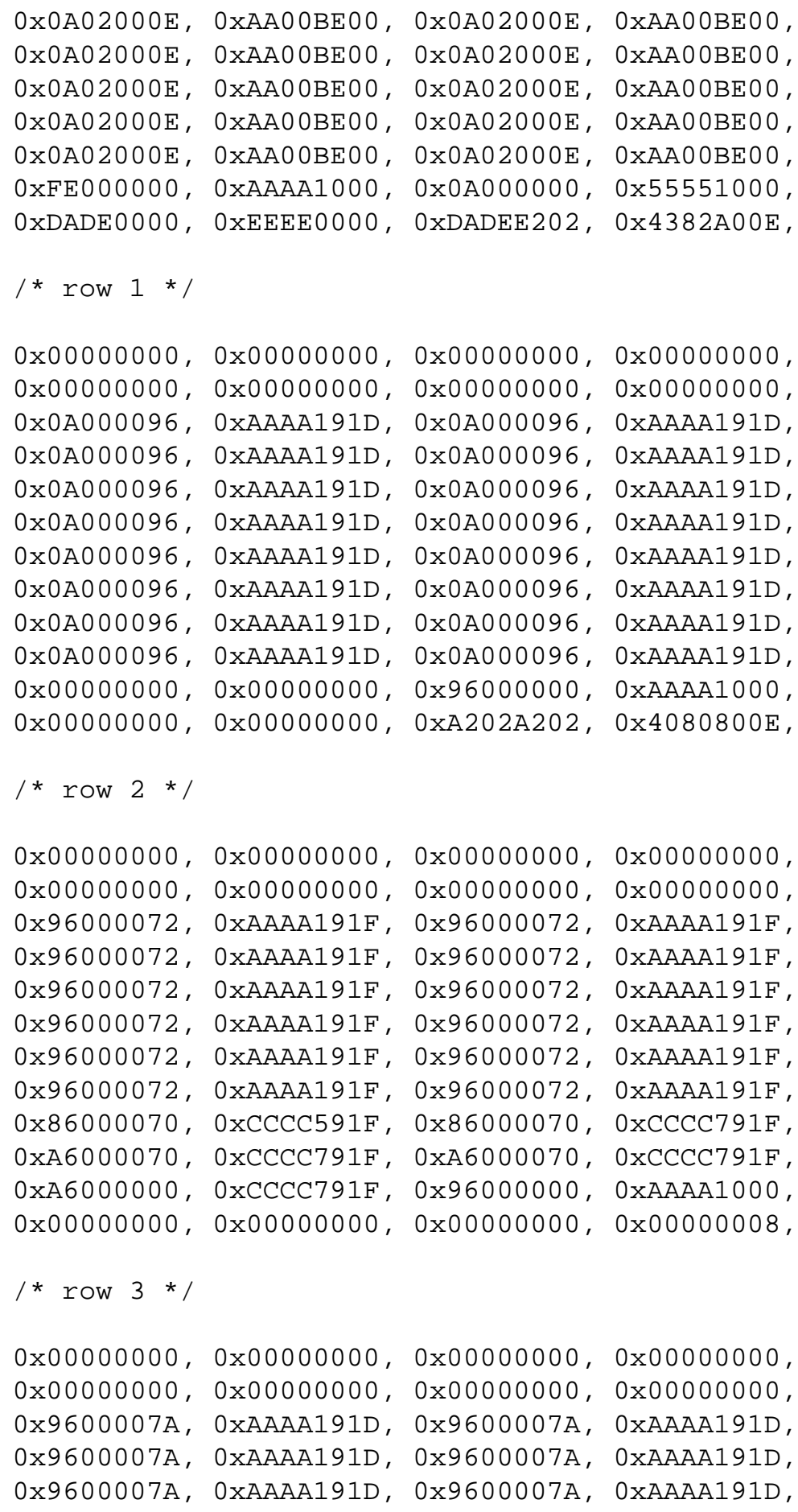




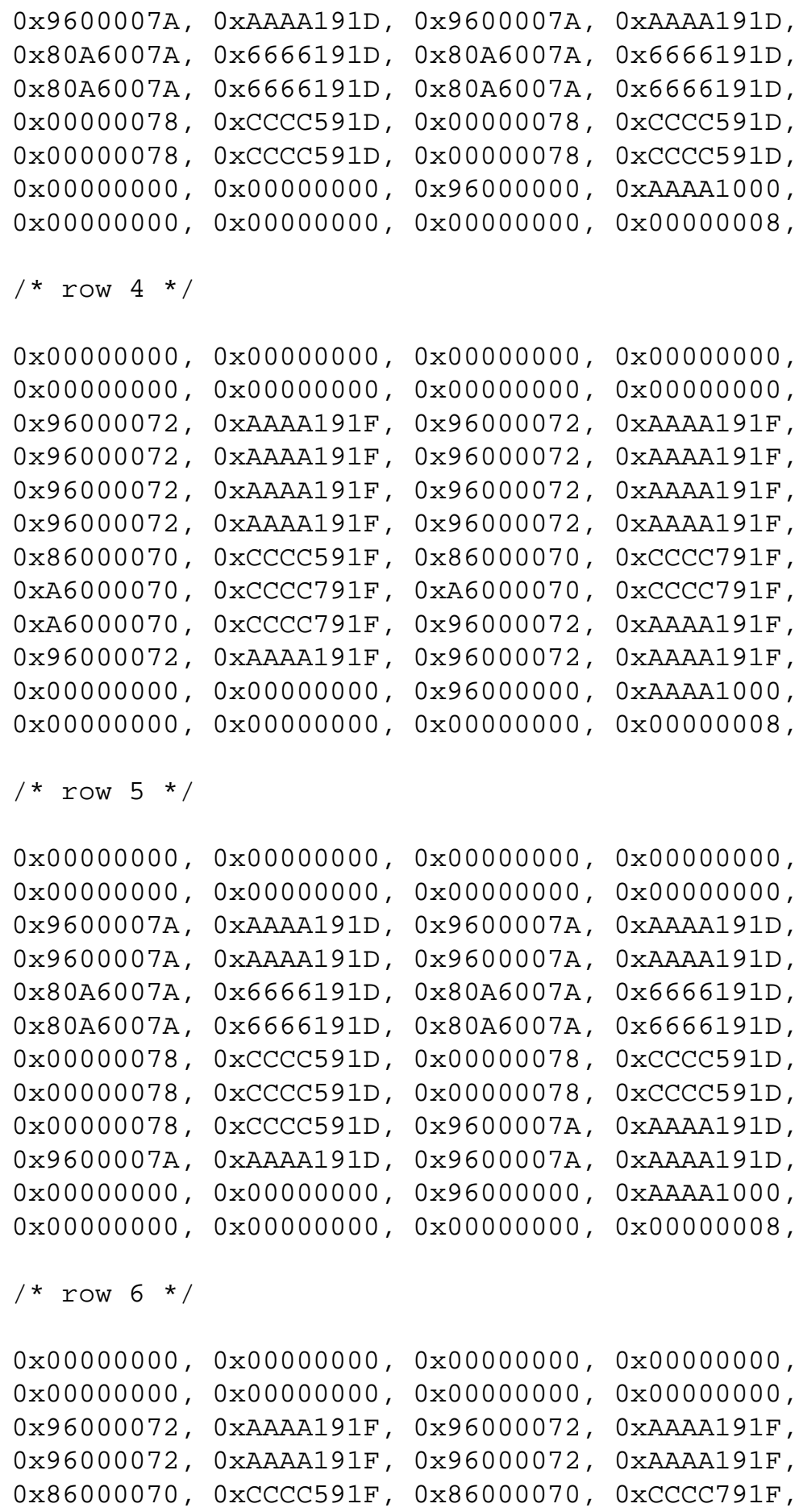




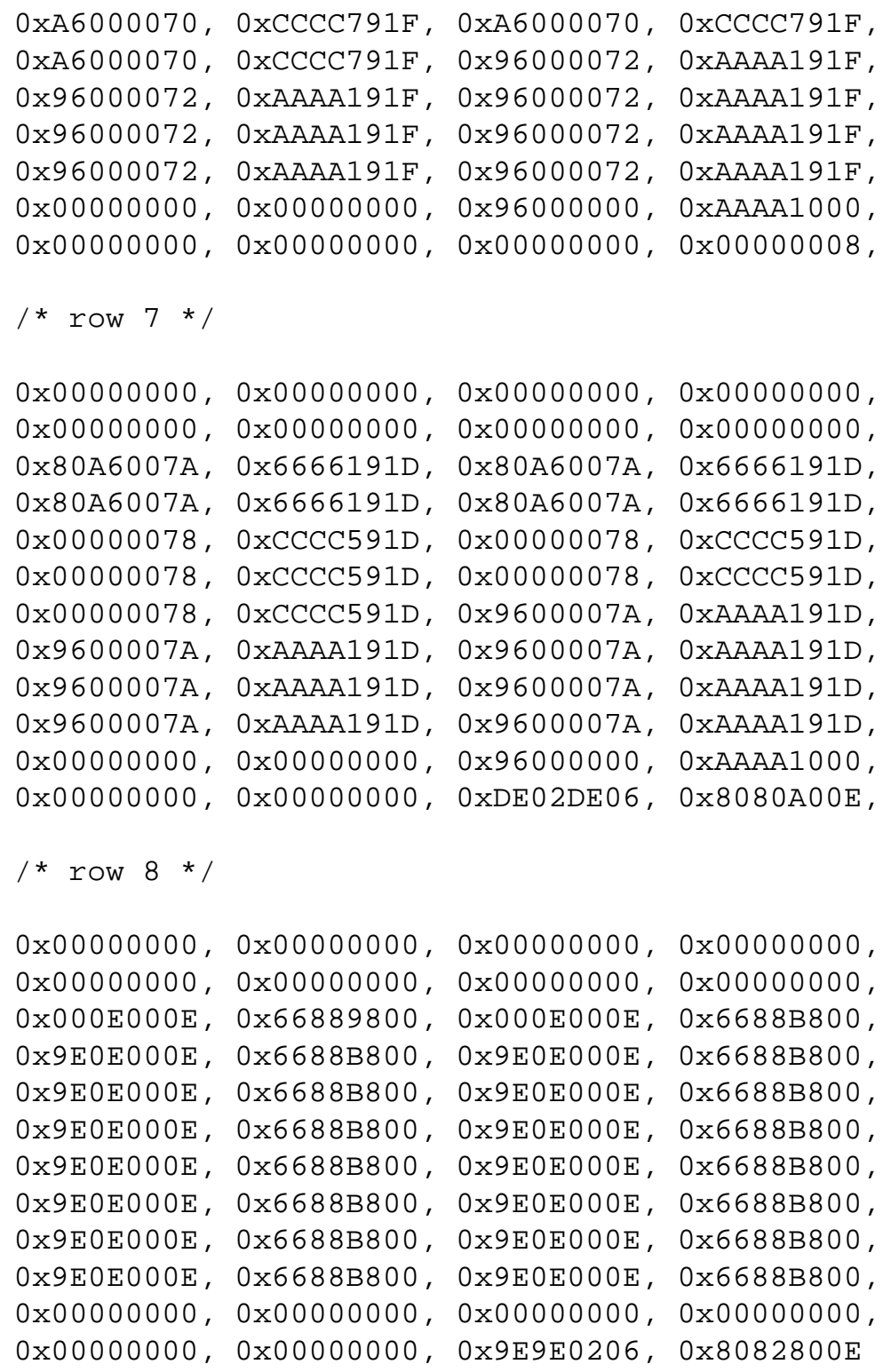

\section{B Example Properties}

These are some of the actual SMV language properties checked for the examples. The entire translated confi gurations were too large to $\mathrm{fi} t$ in the report, but are available at:

http://www.cs.unm.edu/ slugboy/papers/tr_fpl_03/tr_fpl_03.shtml

Additional useful information and fi les from John Cochran's Master's thesis are available at: 
http://www.cs.unm.edu/ slugboy/thesis/thesis.shtml

\section{B.1 IM1}

SPEC -- MC

-- the correct initialization of the control path implies correct -- memory synchronization

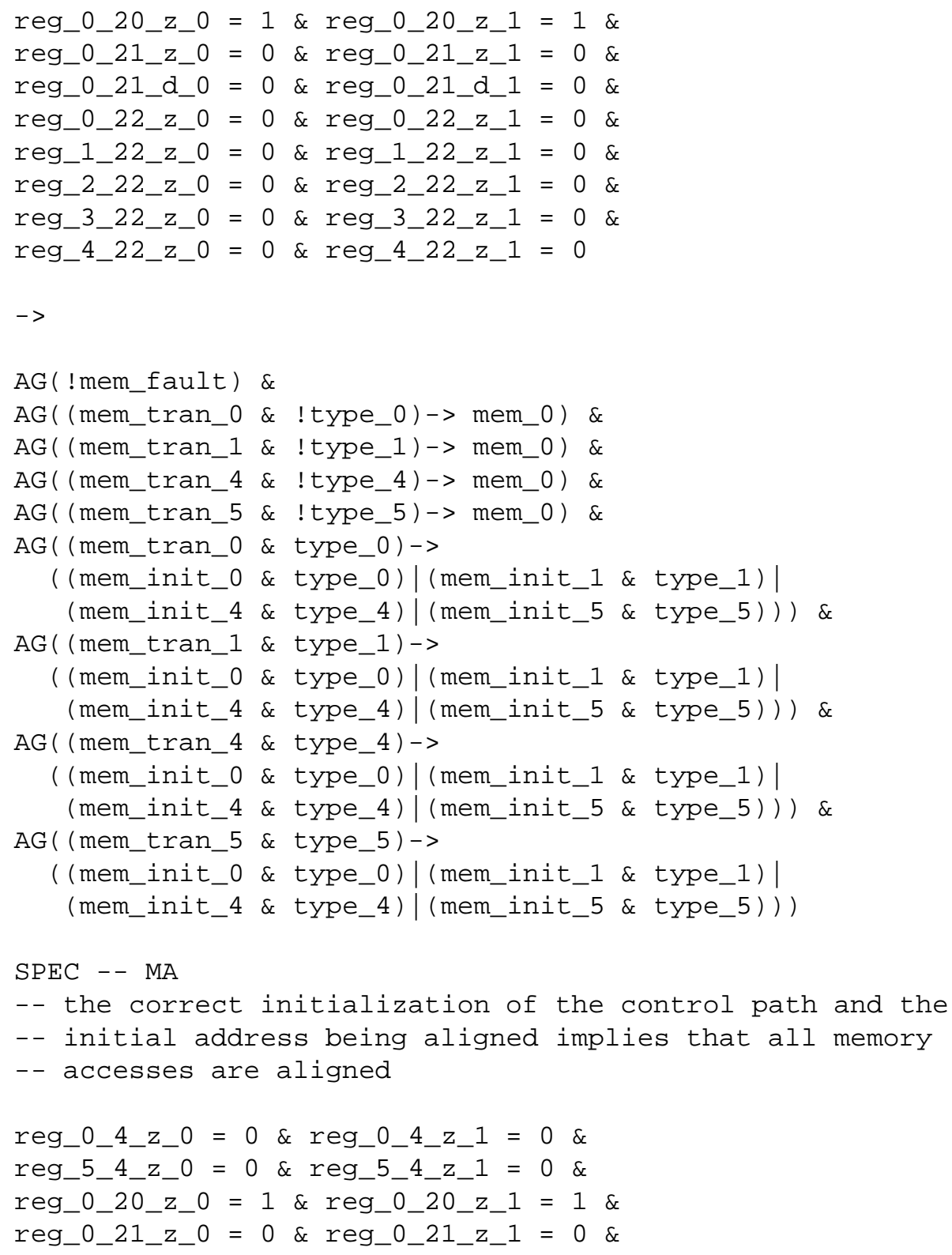




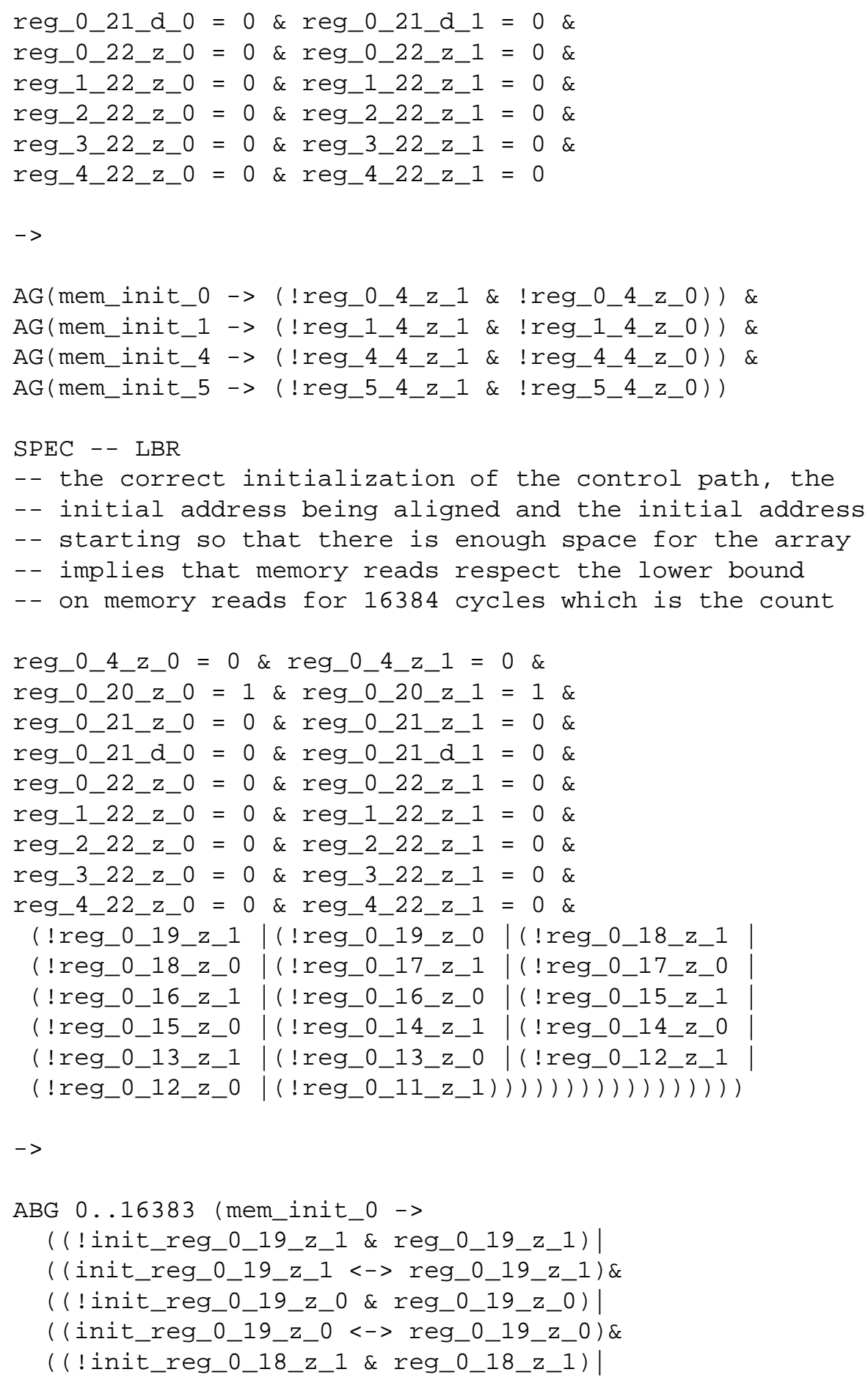




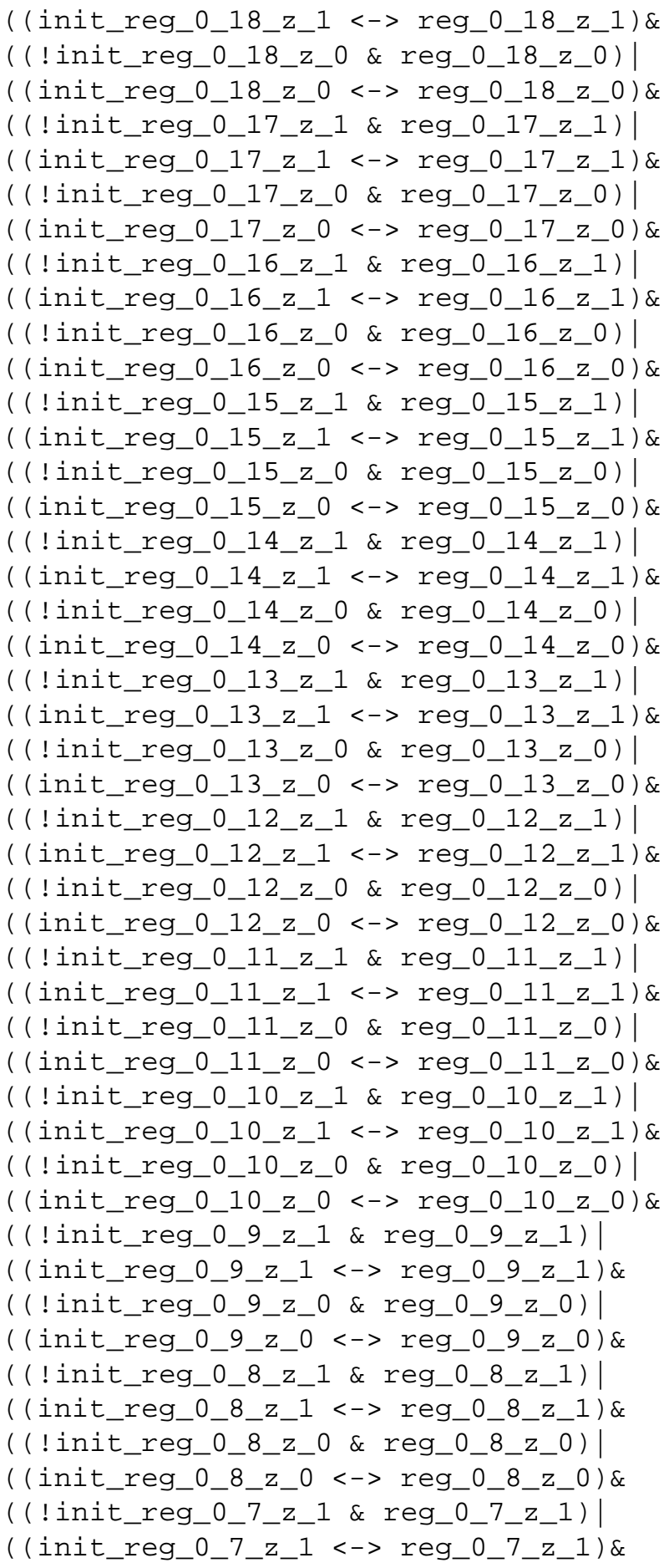




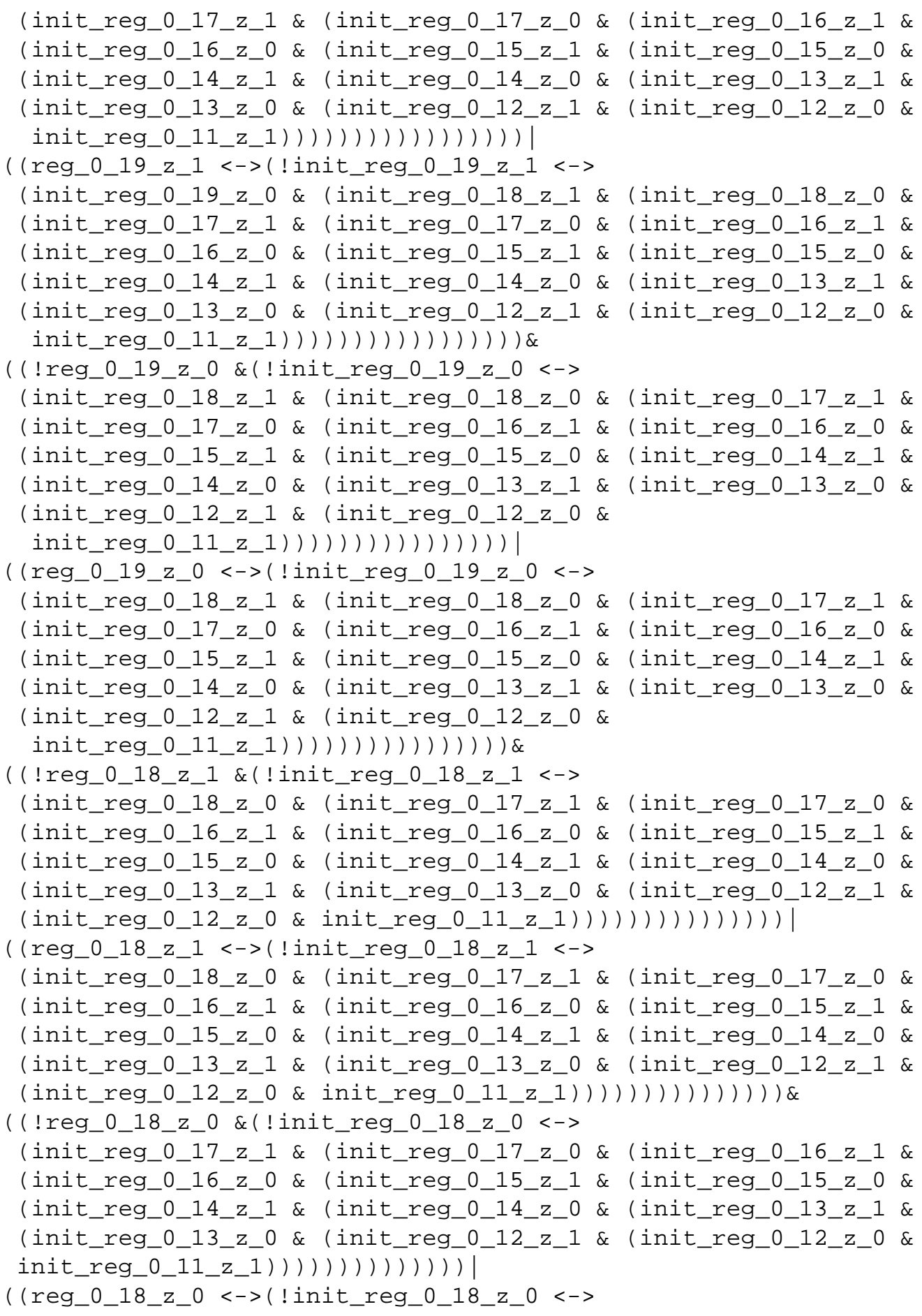




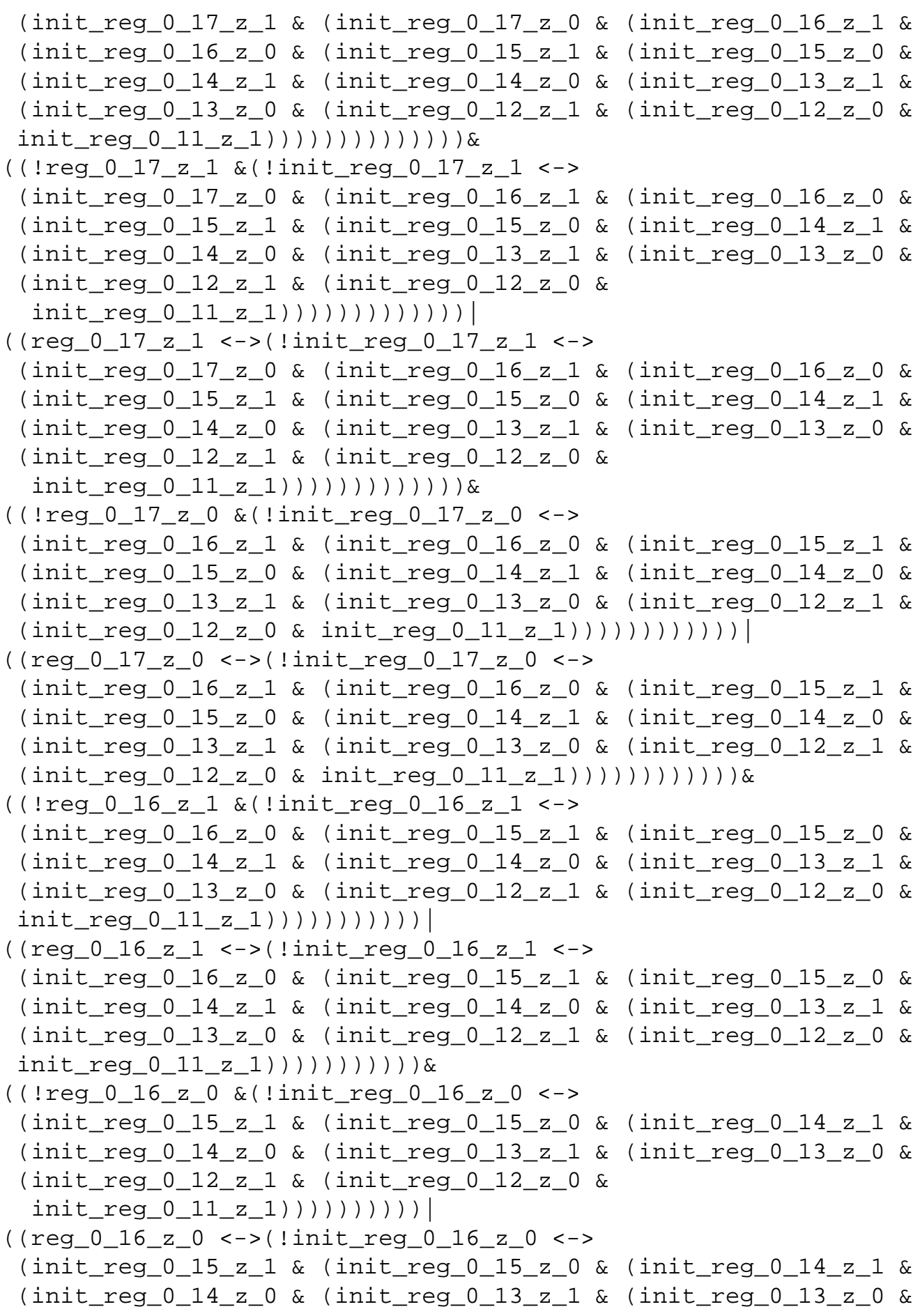




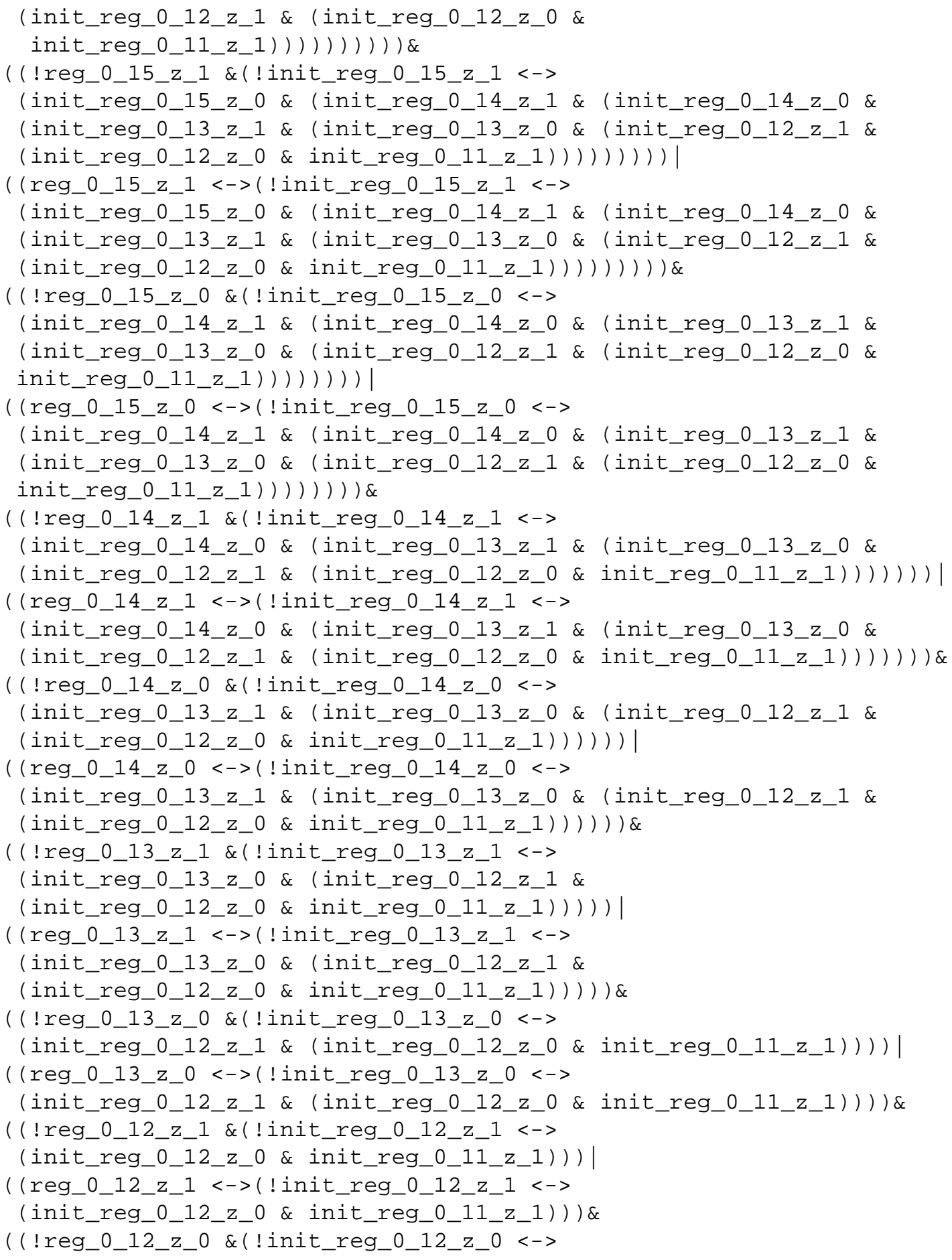




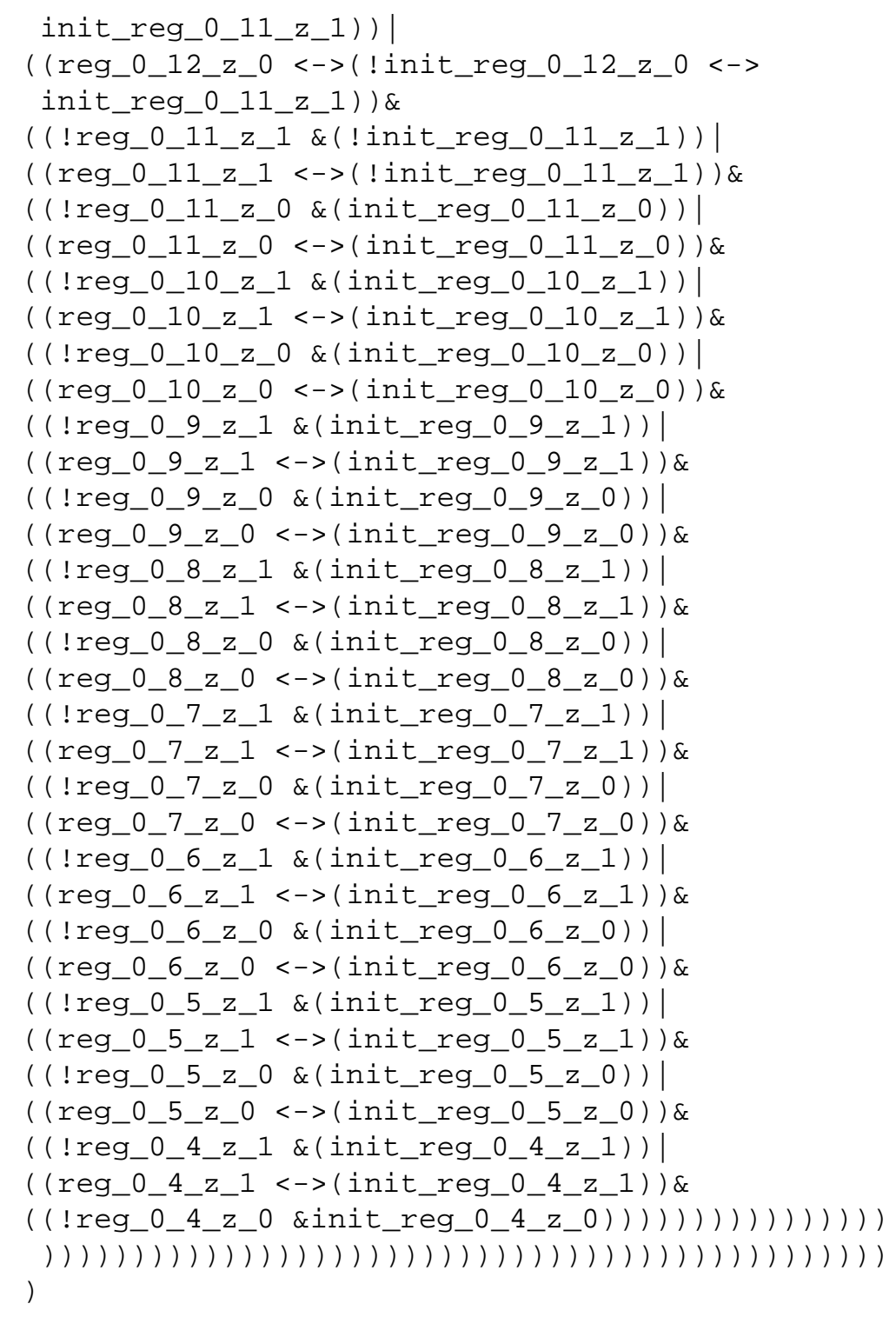

\section{B.2 IM2}

SPEC -- MC

-- the correct initialization of the control path implies correct -- memory synchronization

reg_0_20_z_0 $=1$ \& reg_0_20_z_1 $=1$ \& 


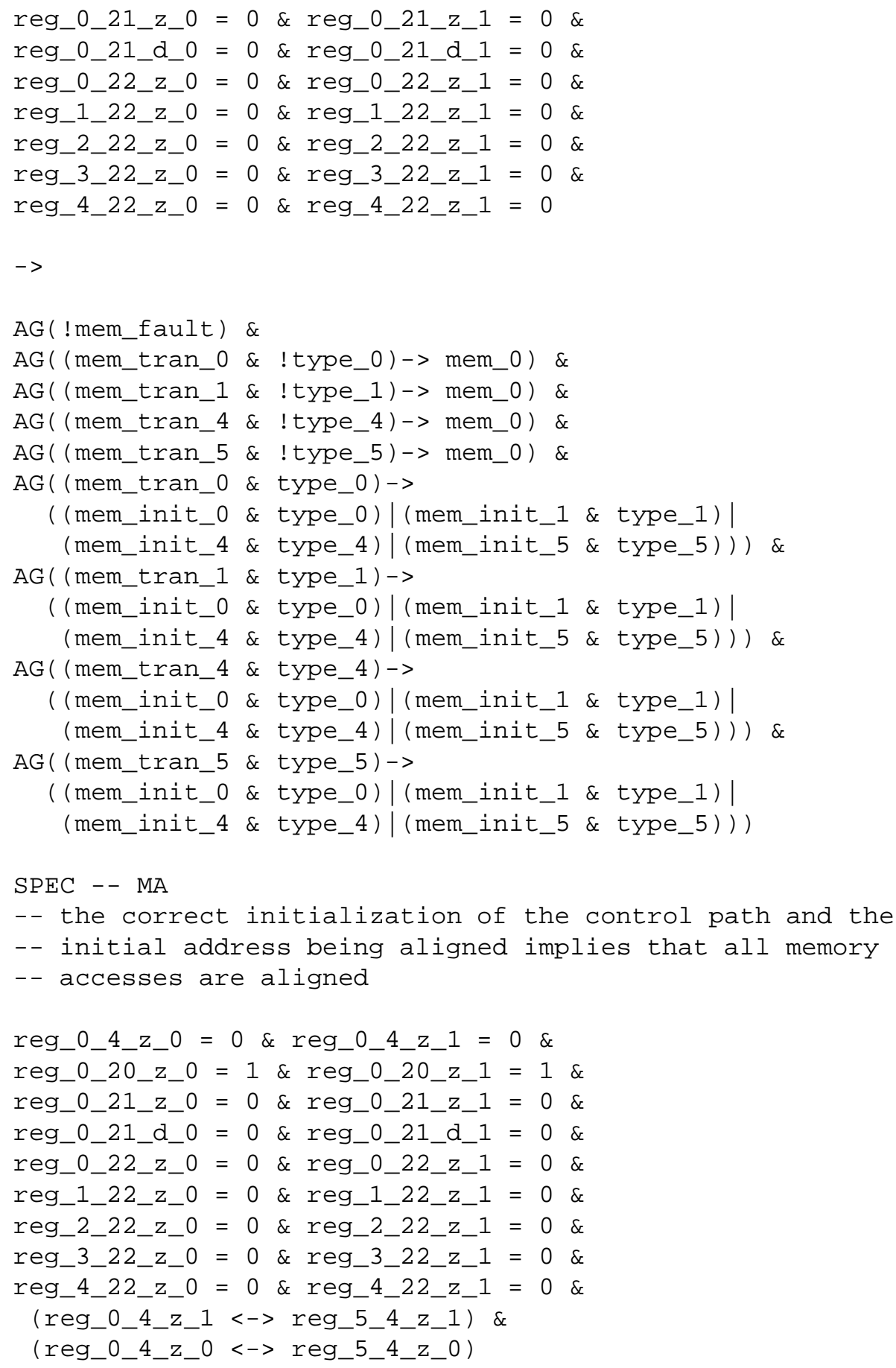




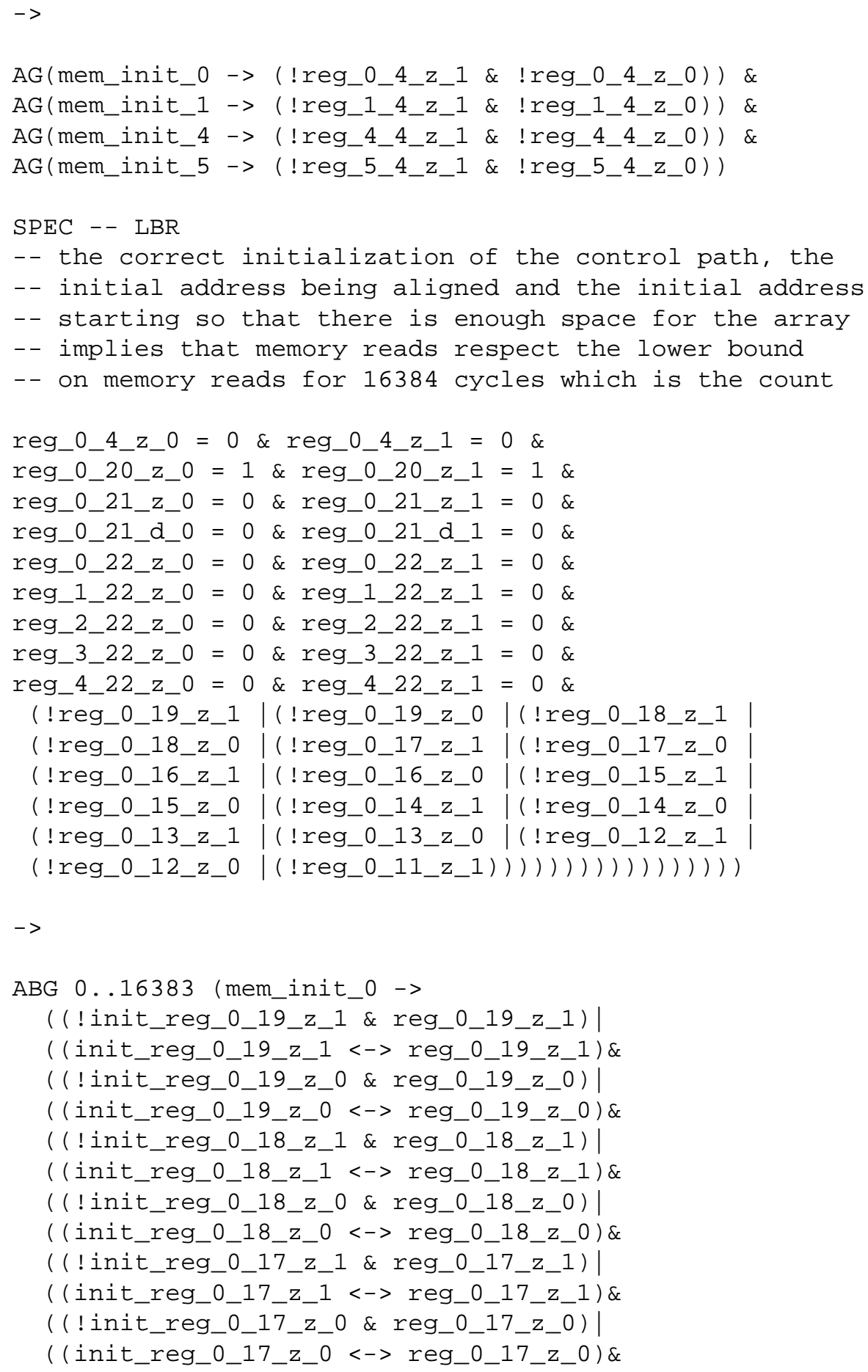




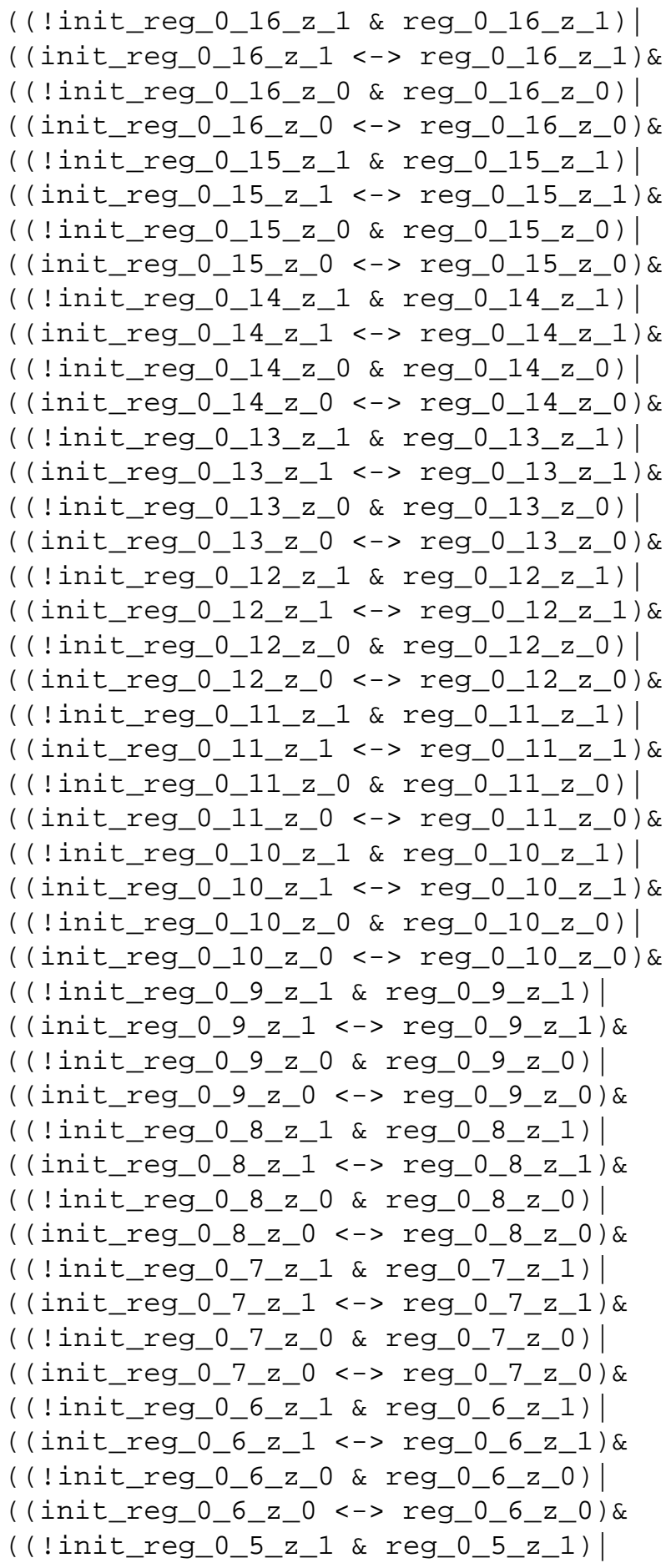




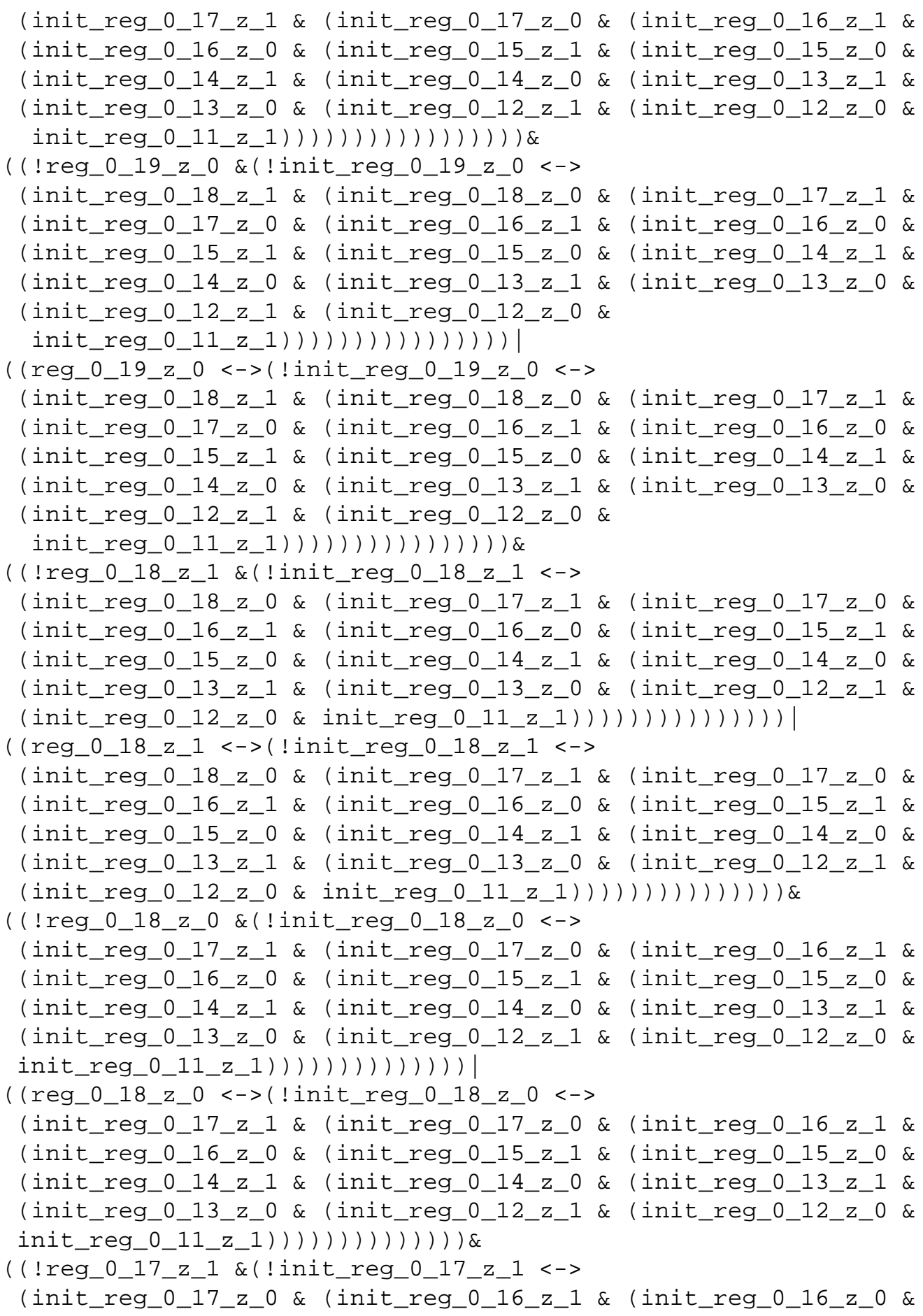




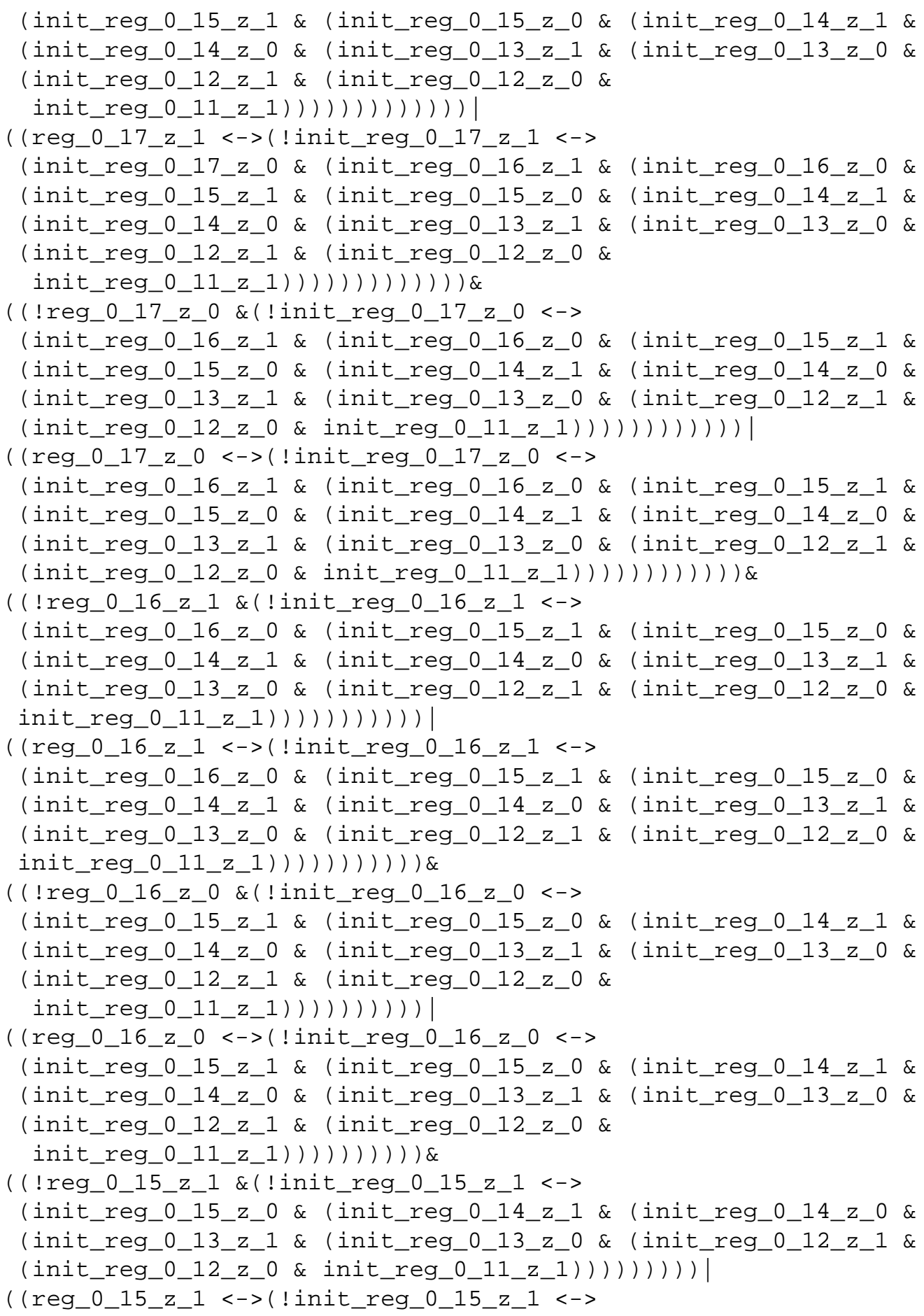




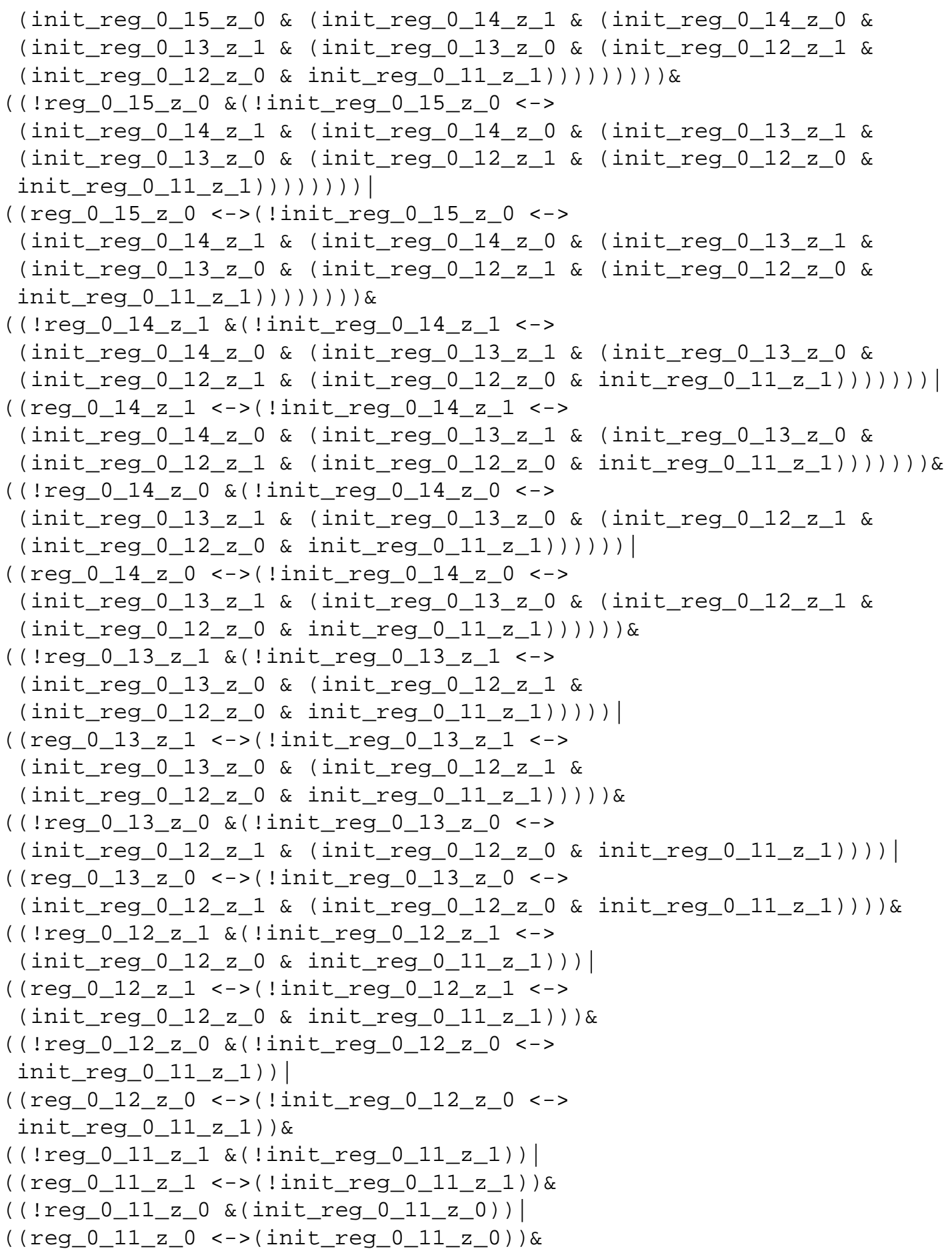




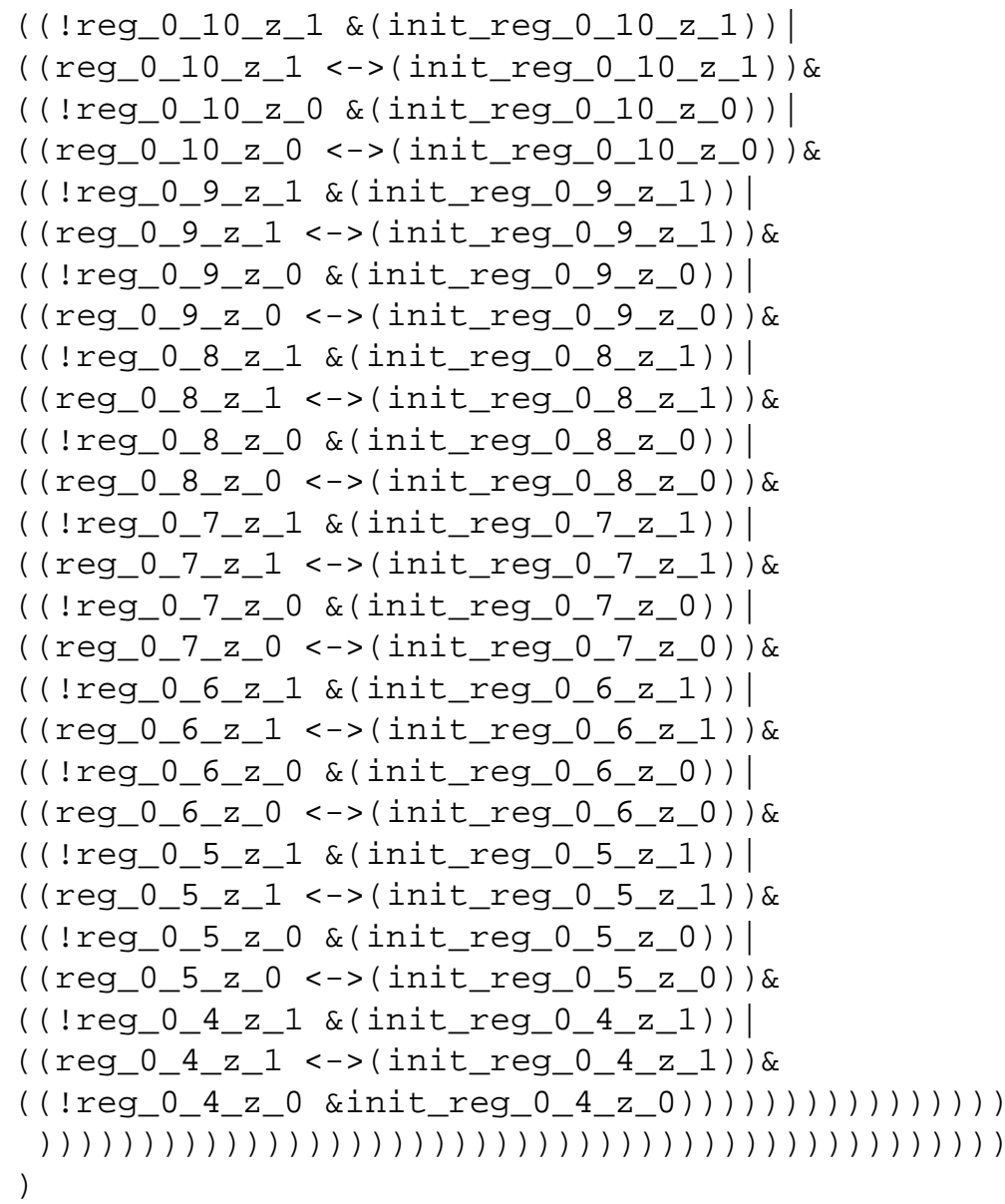




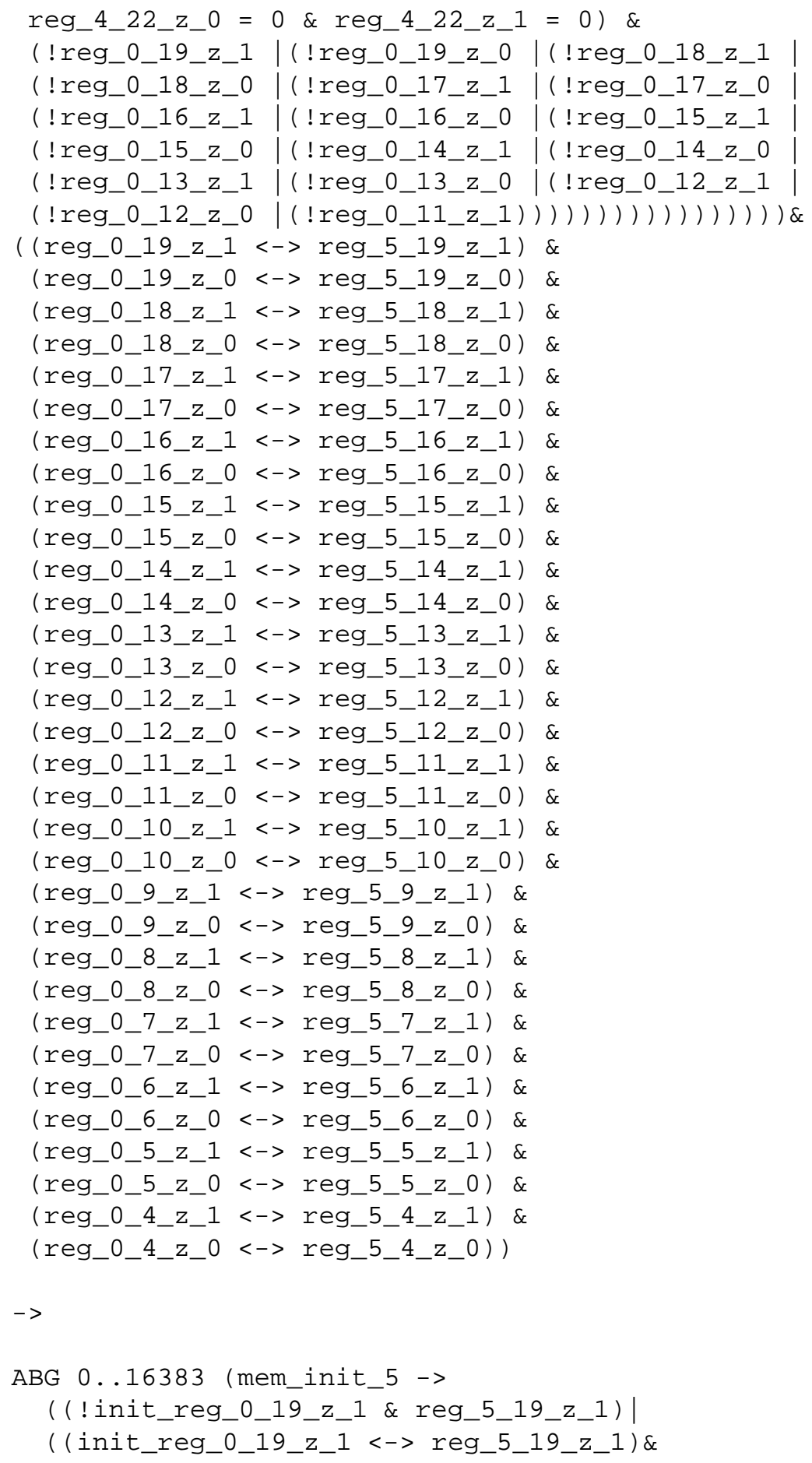




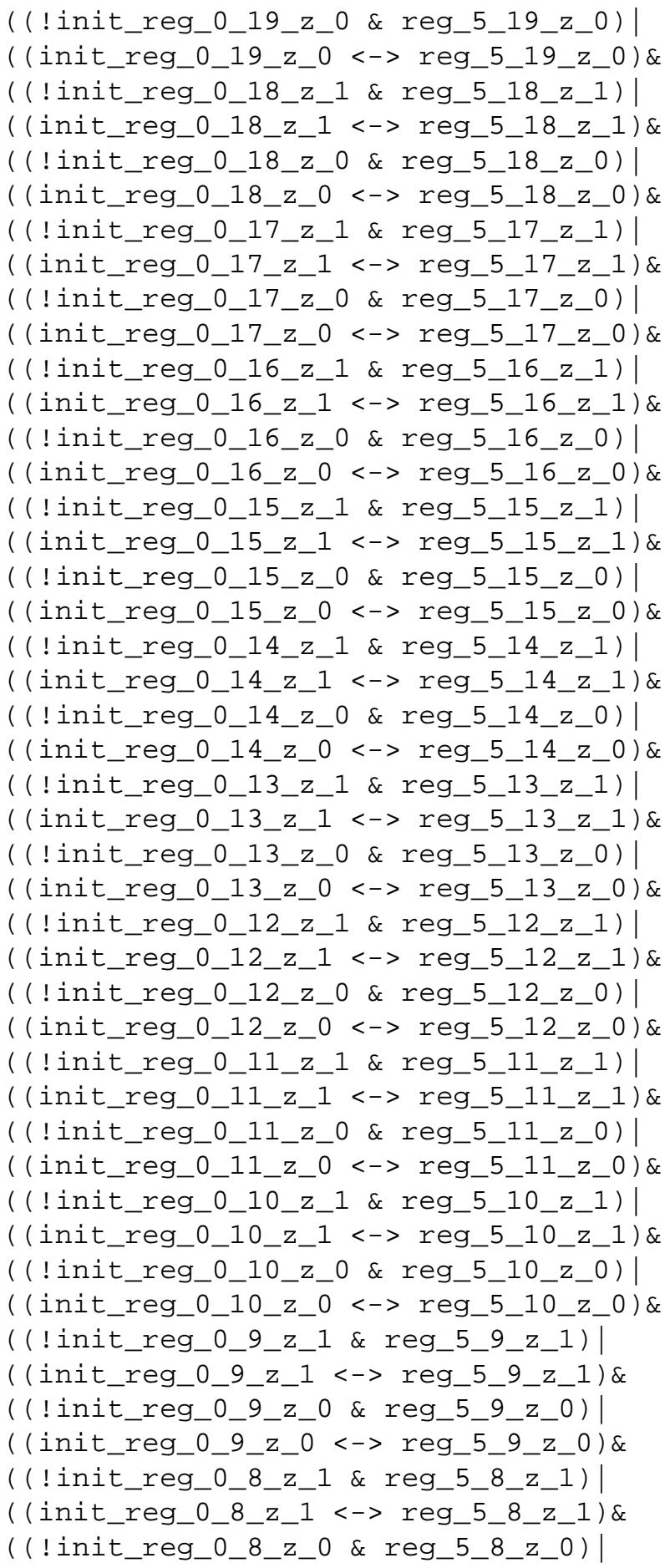




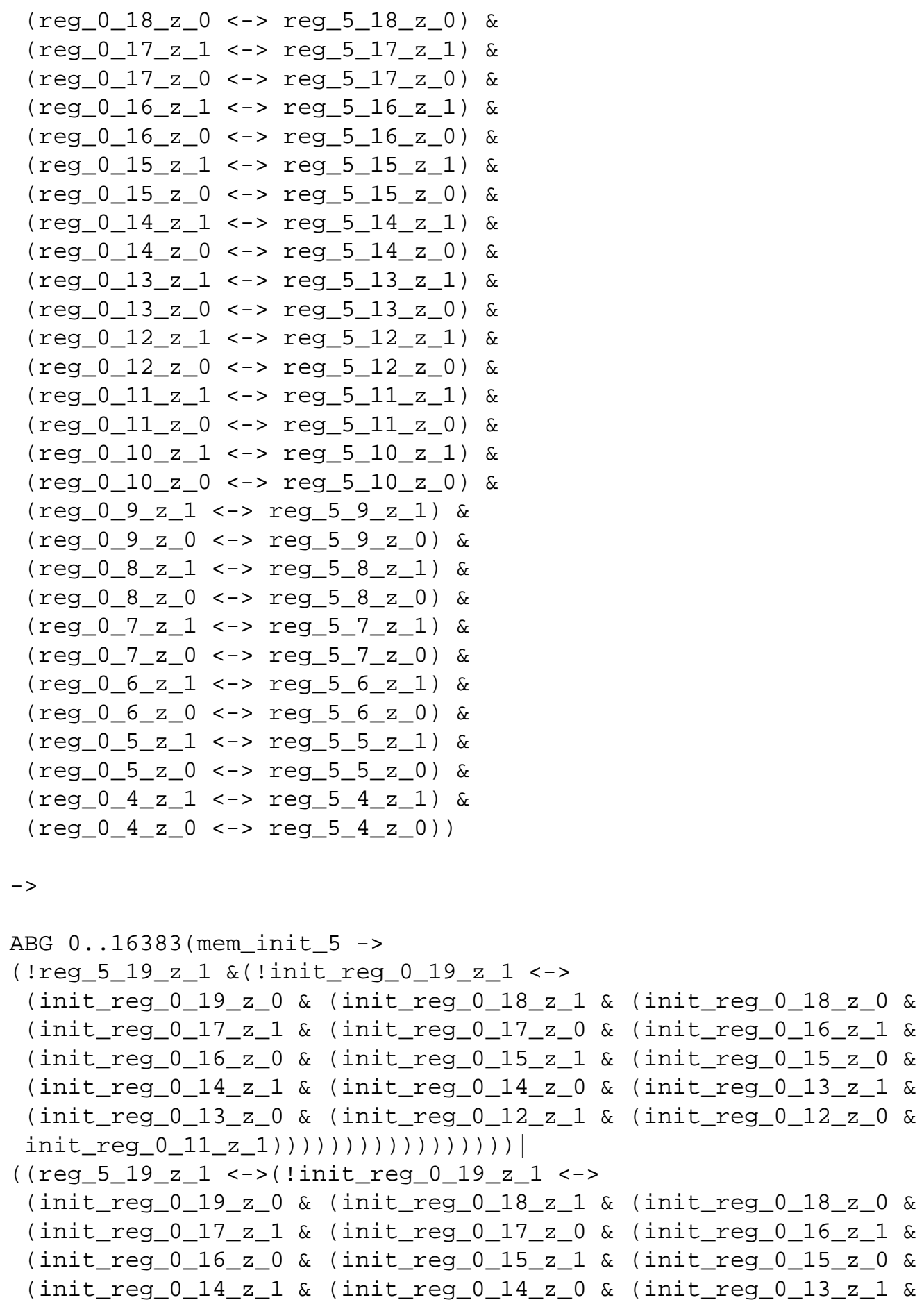




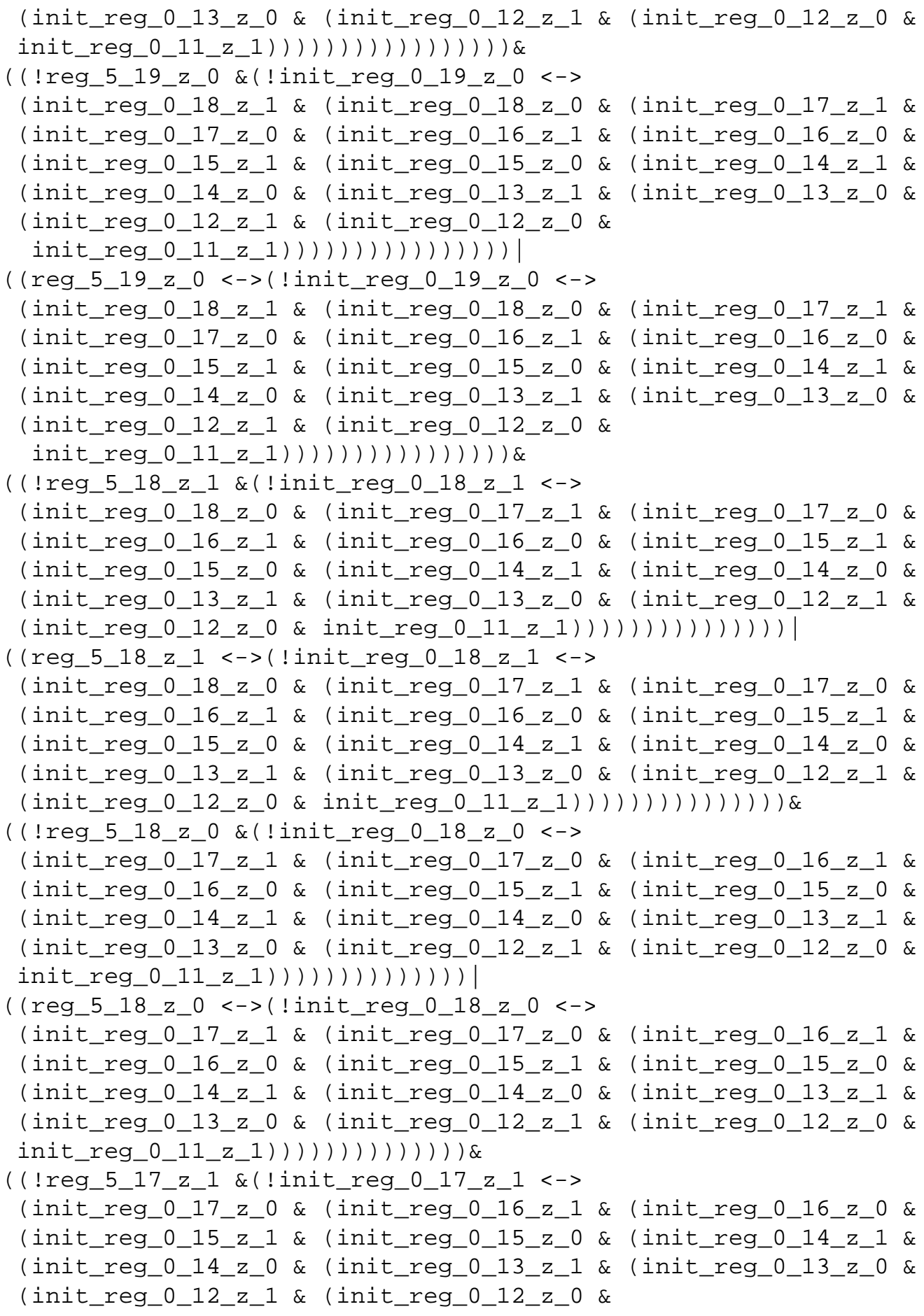




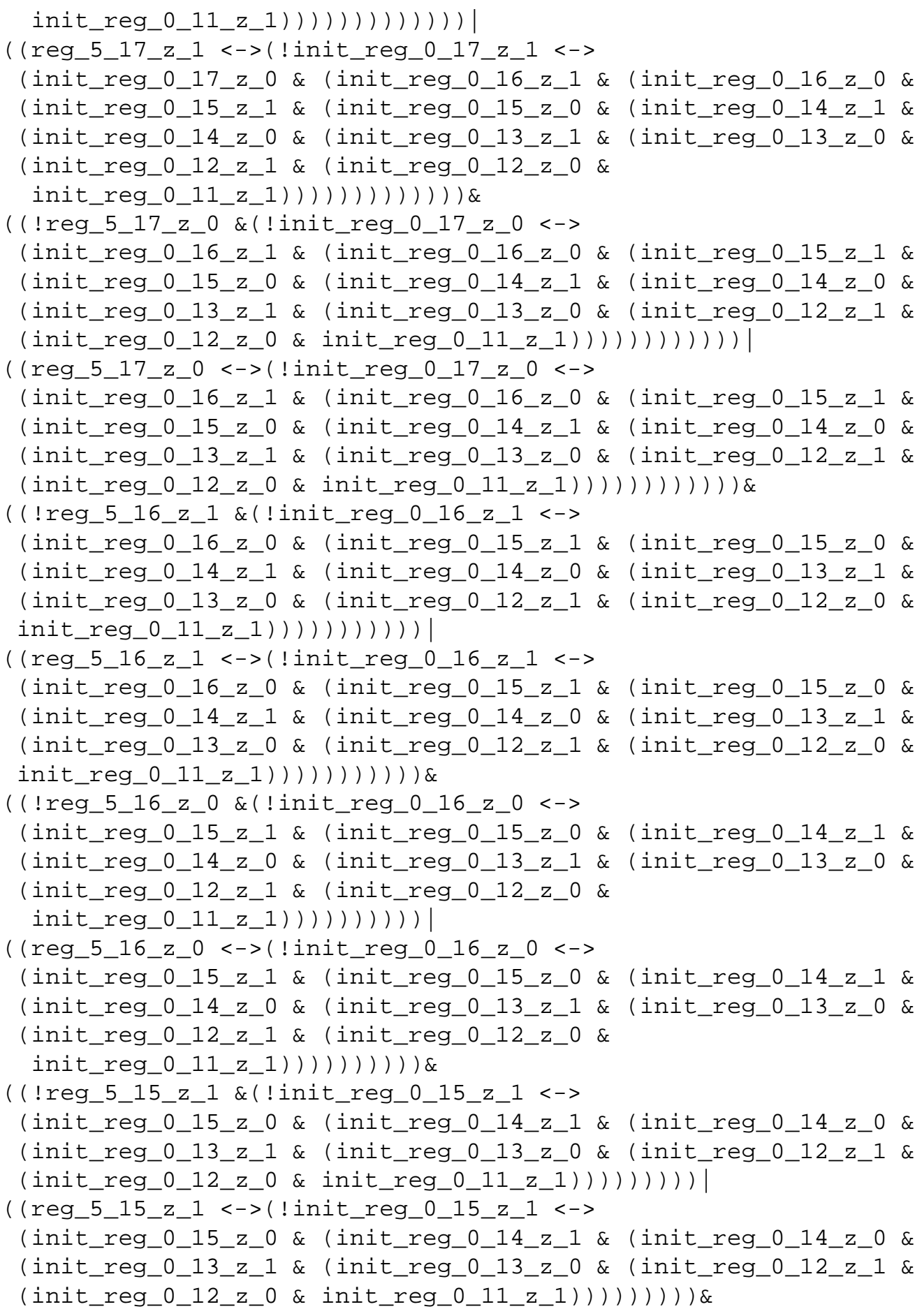




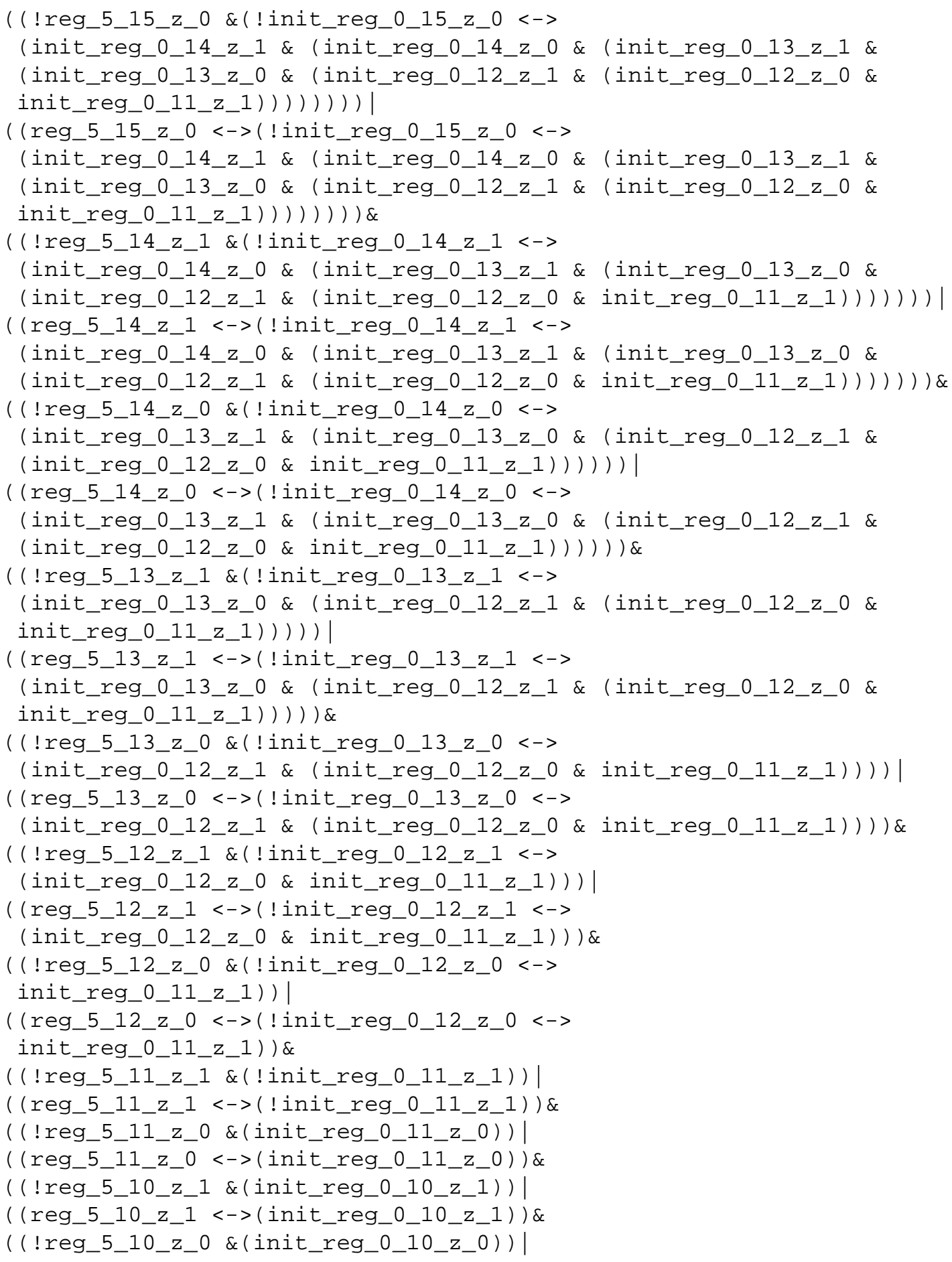




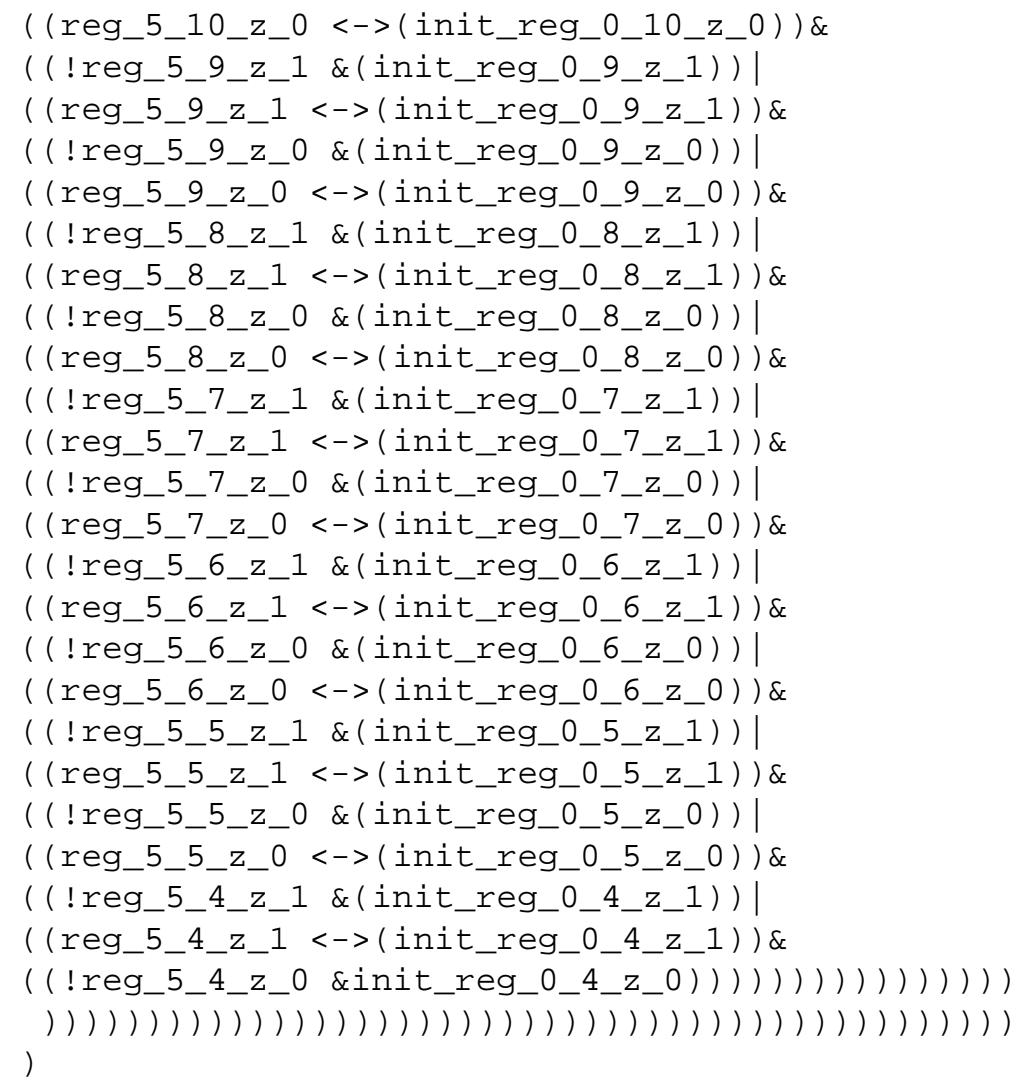

\section{B.3 IM3}

SPEC -- MC

-- the correct initialization of the control path implies correct -- memory synchronization

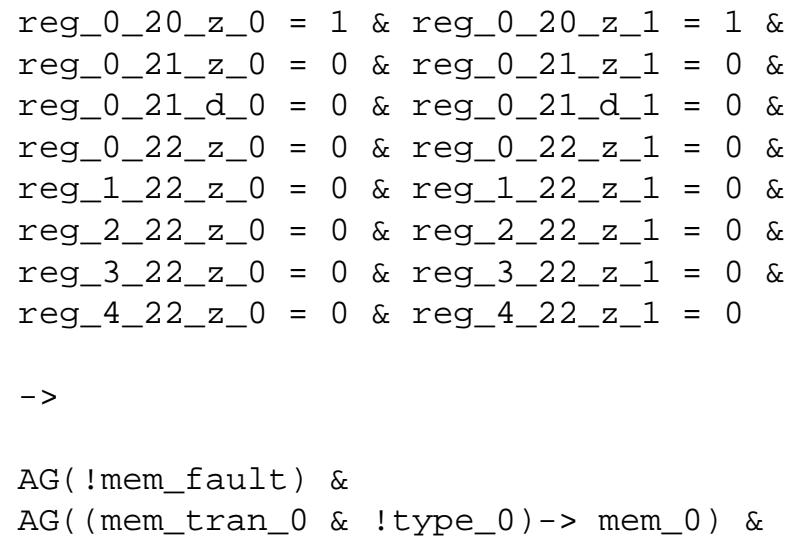




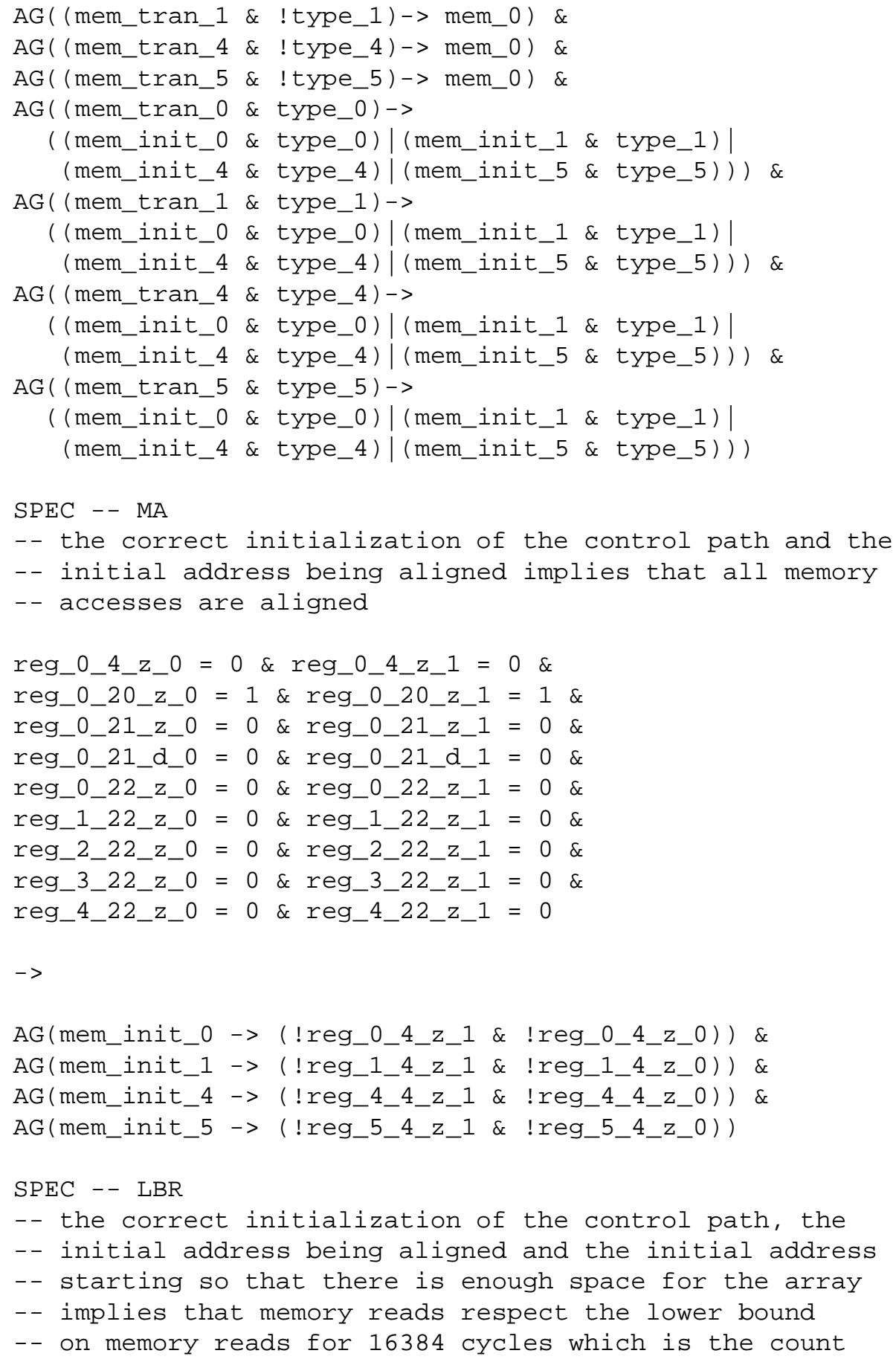




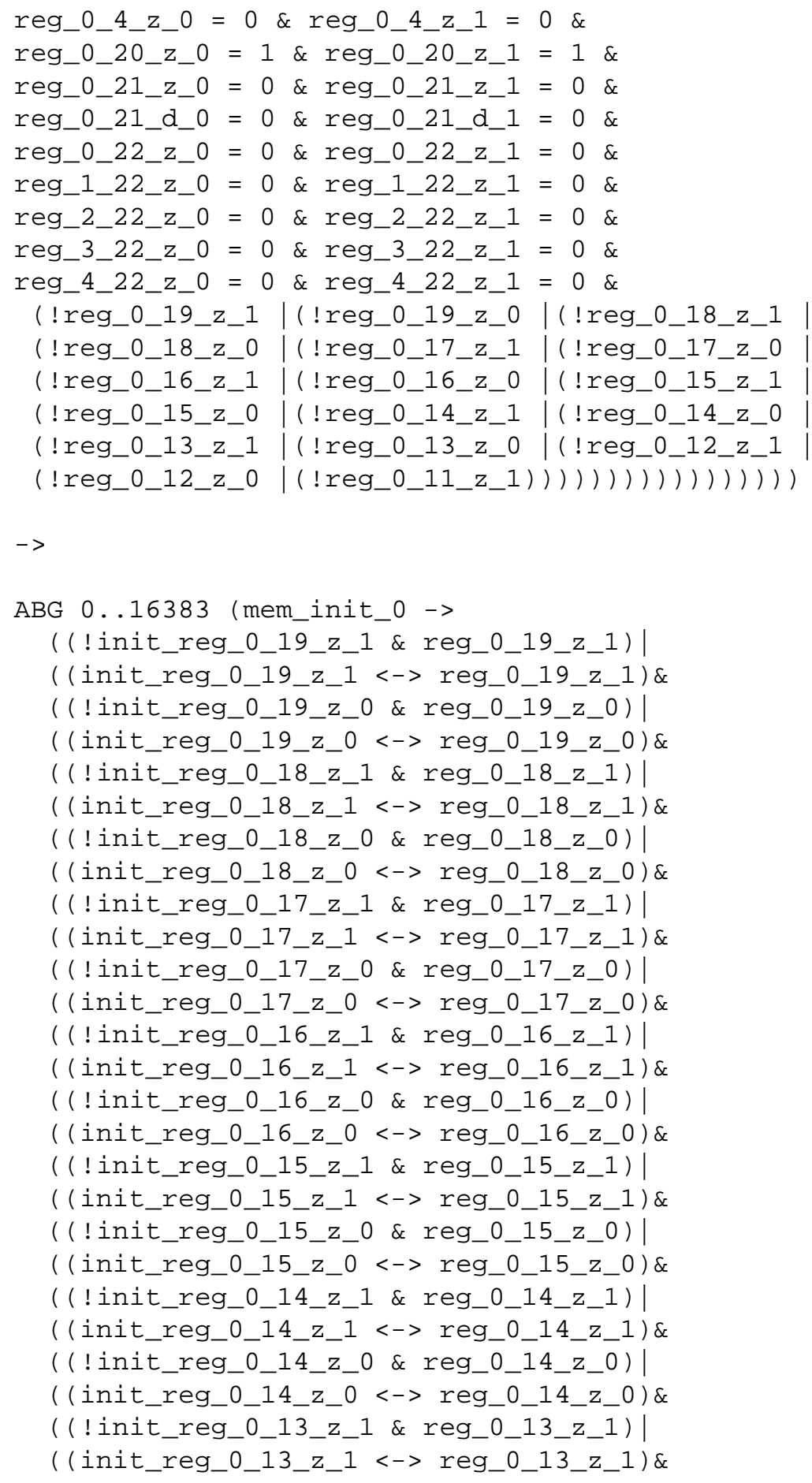




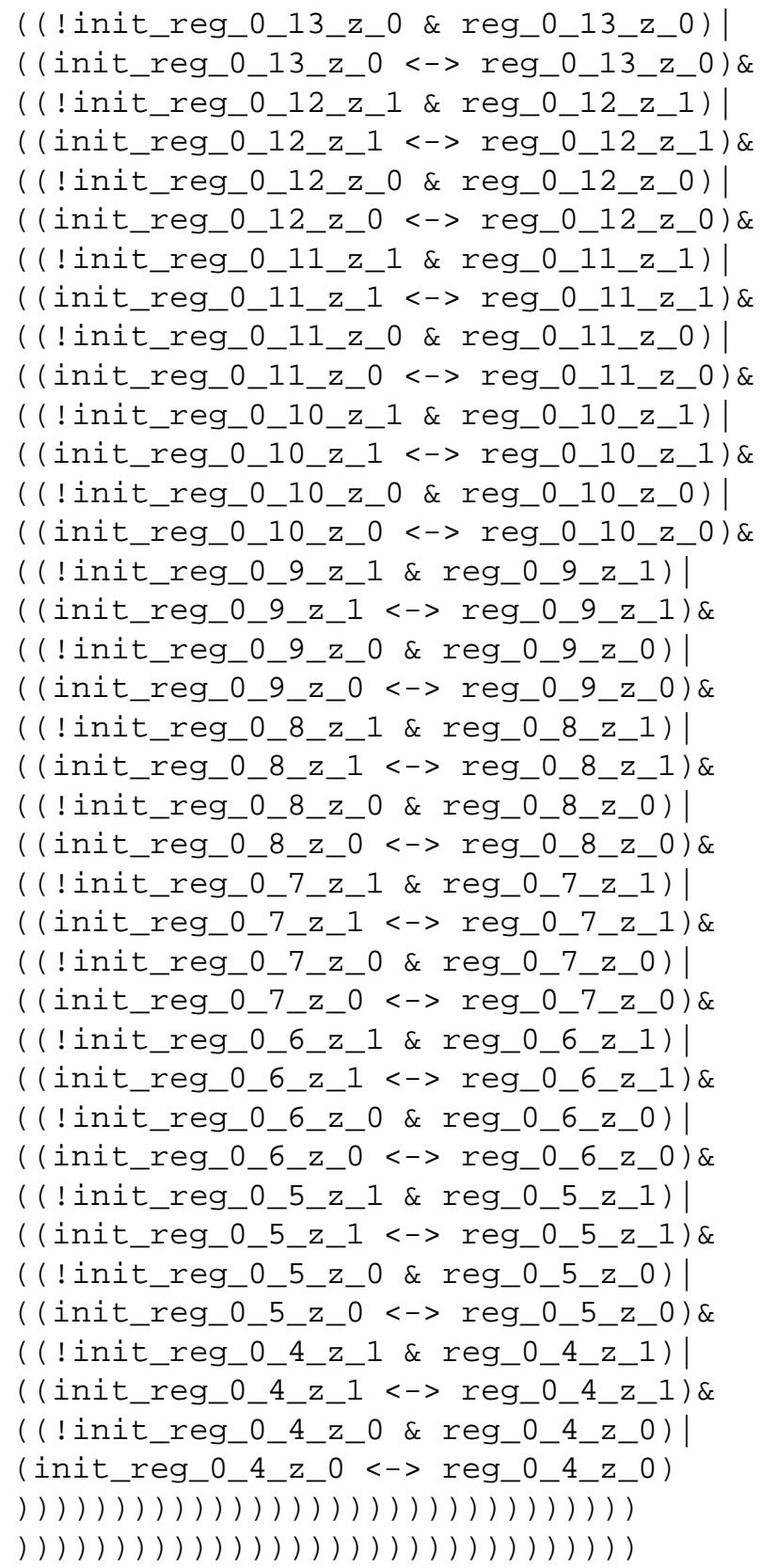


-- implies that memory reads respect the upper bound -- on memory reads for 16384 cycles which is the count

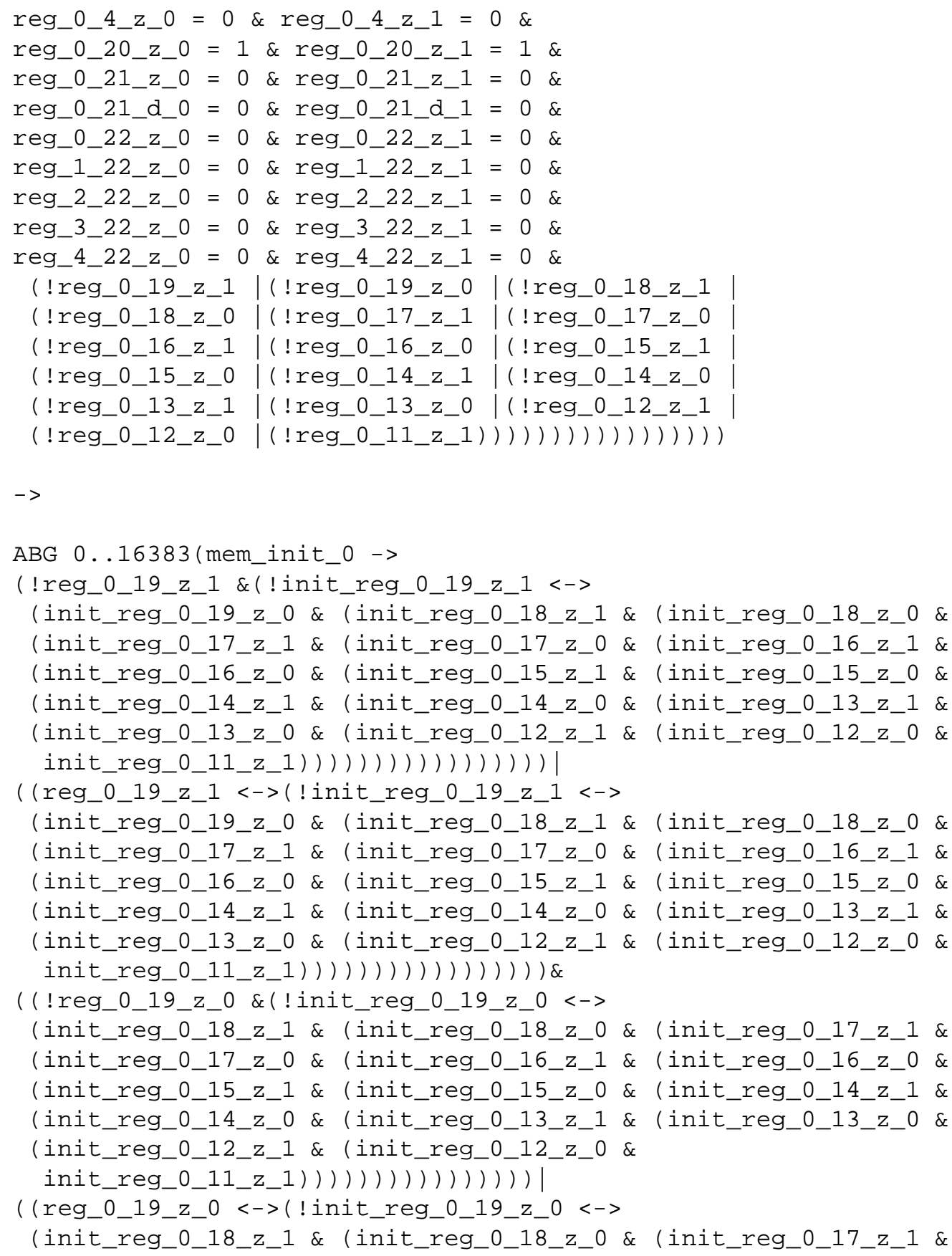




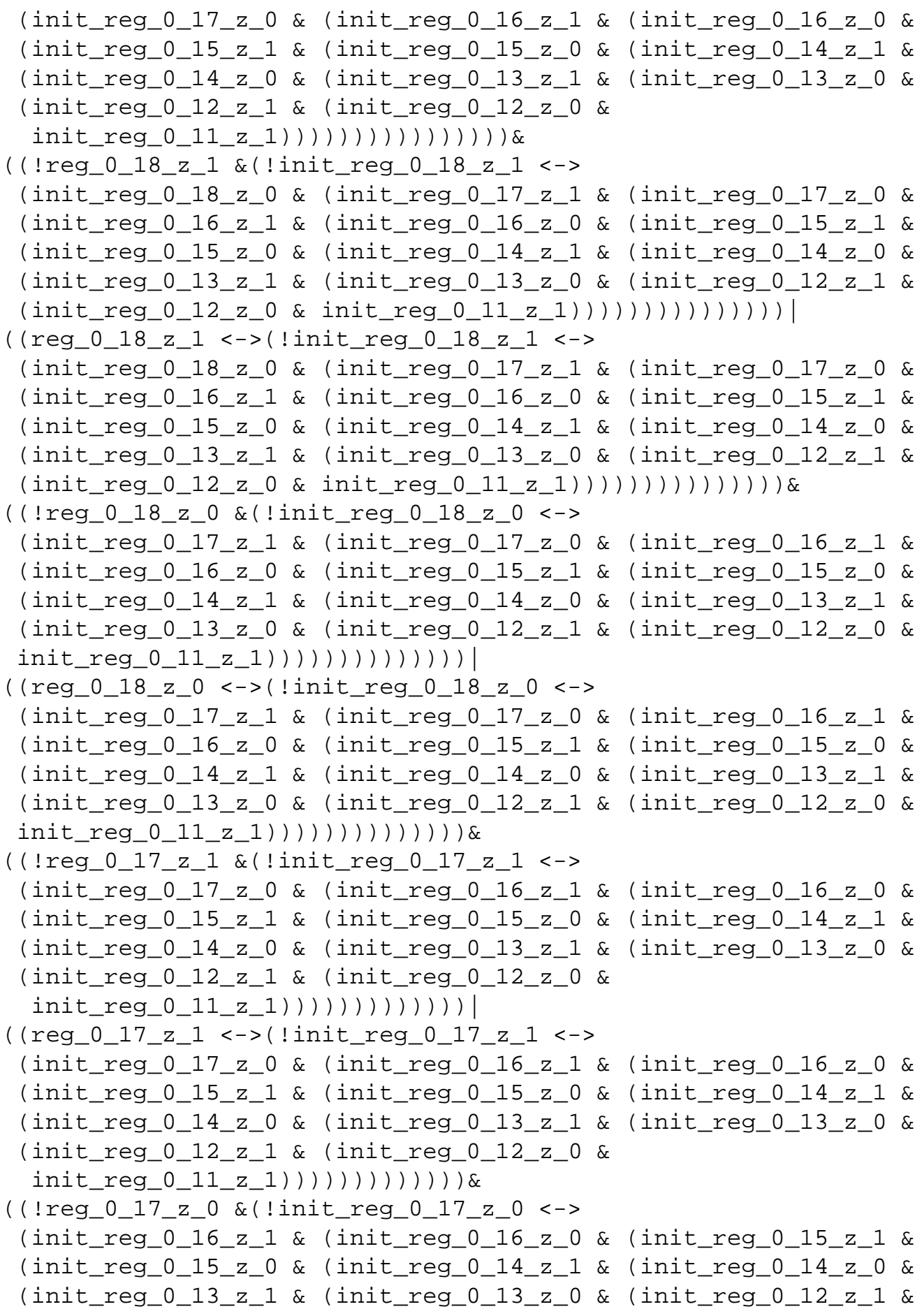




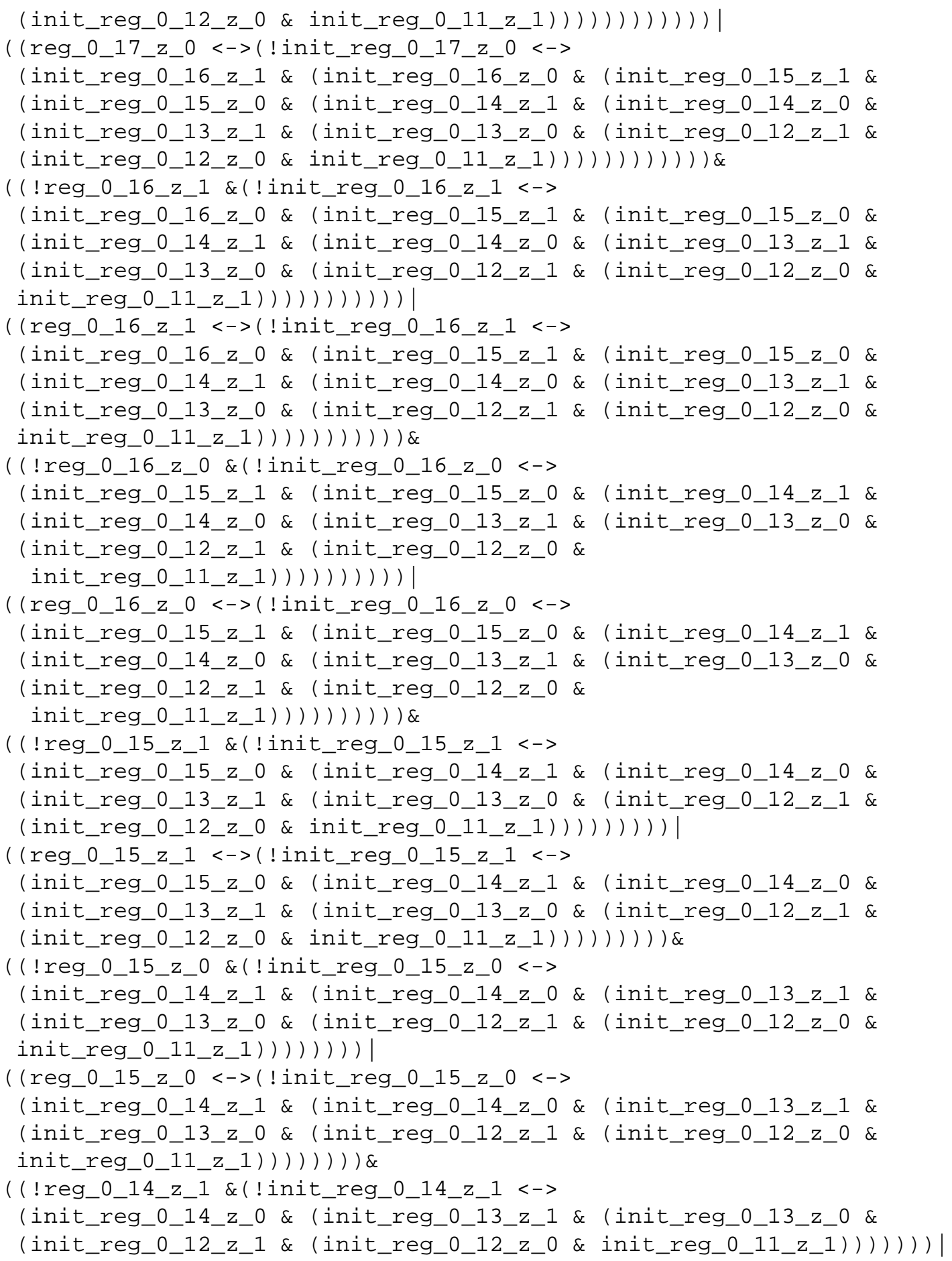




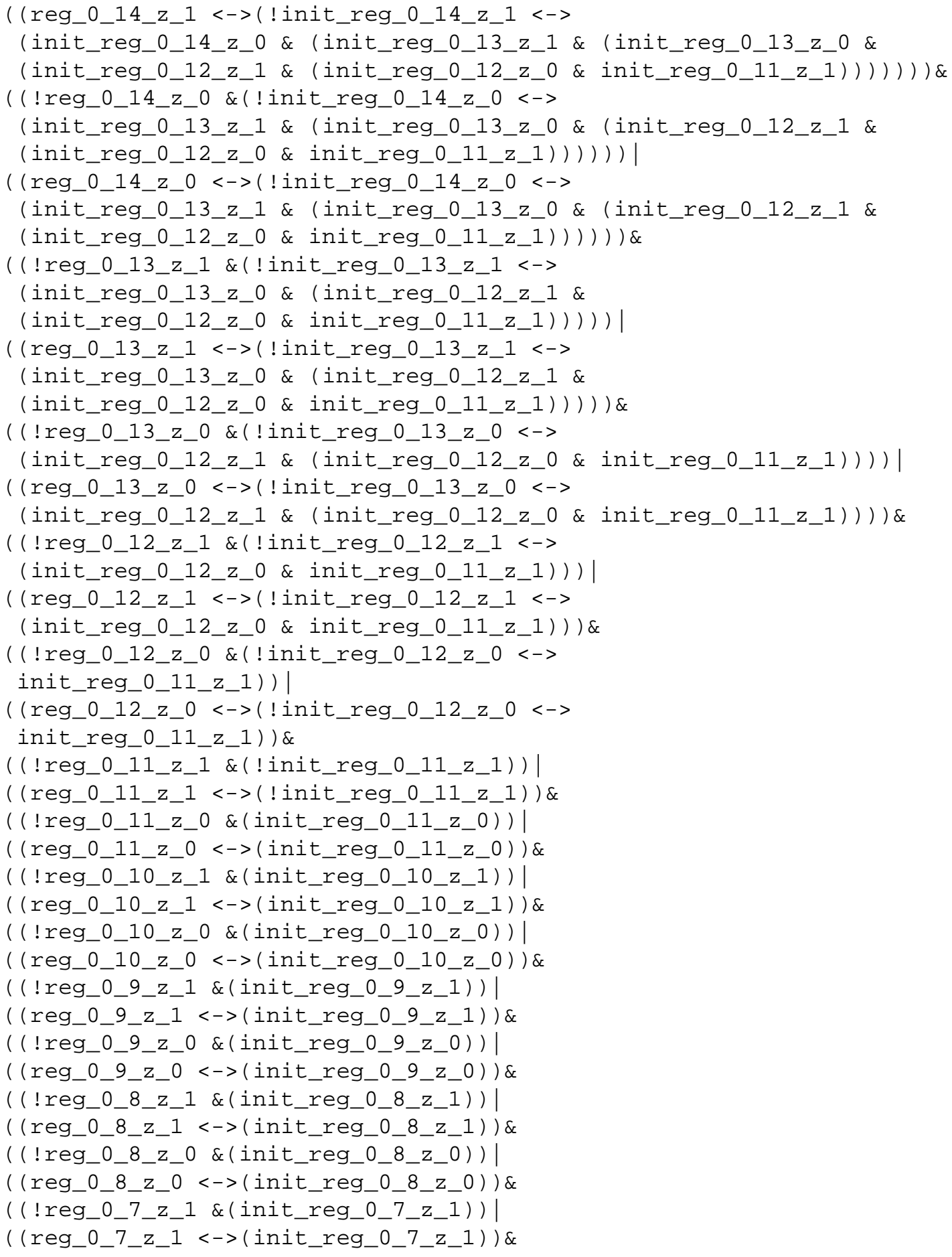




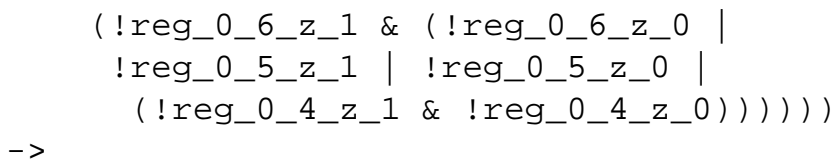




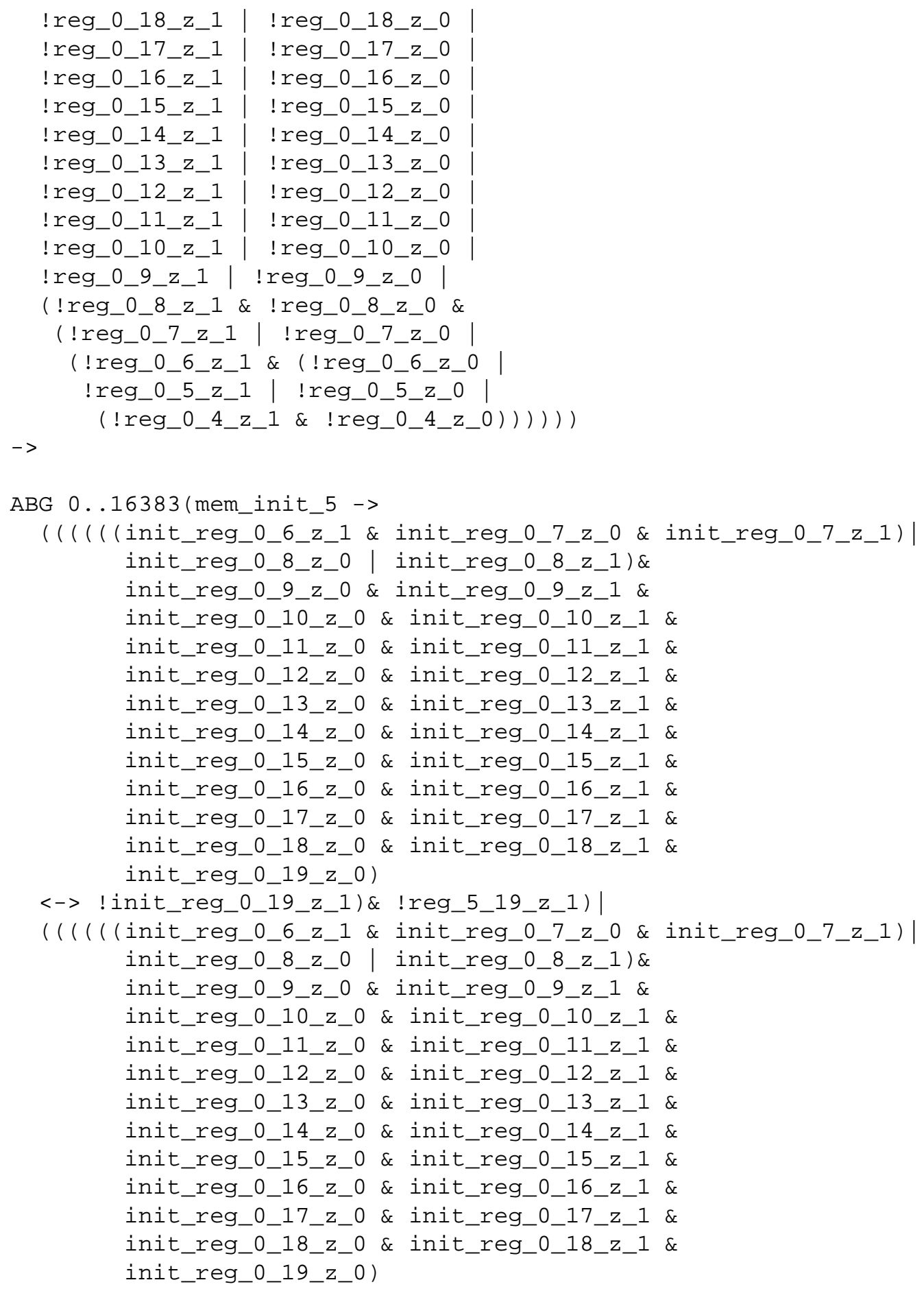




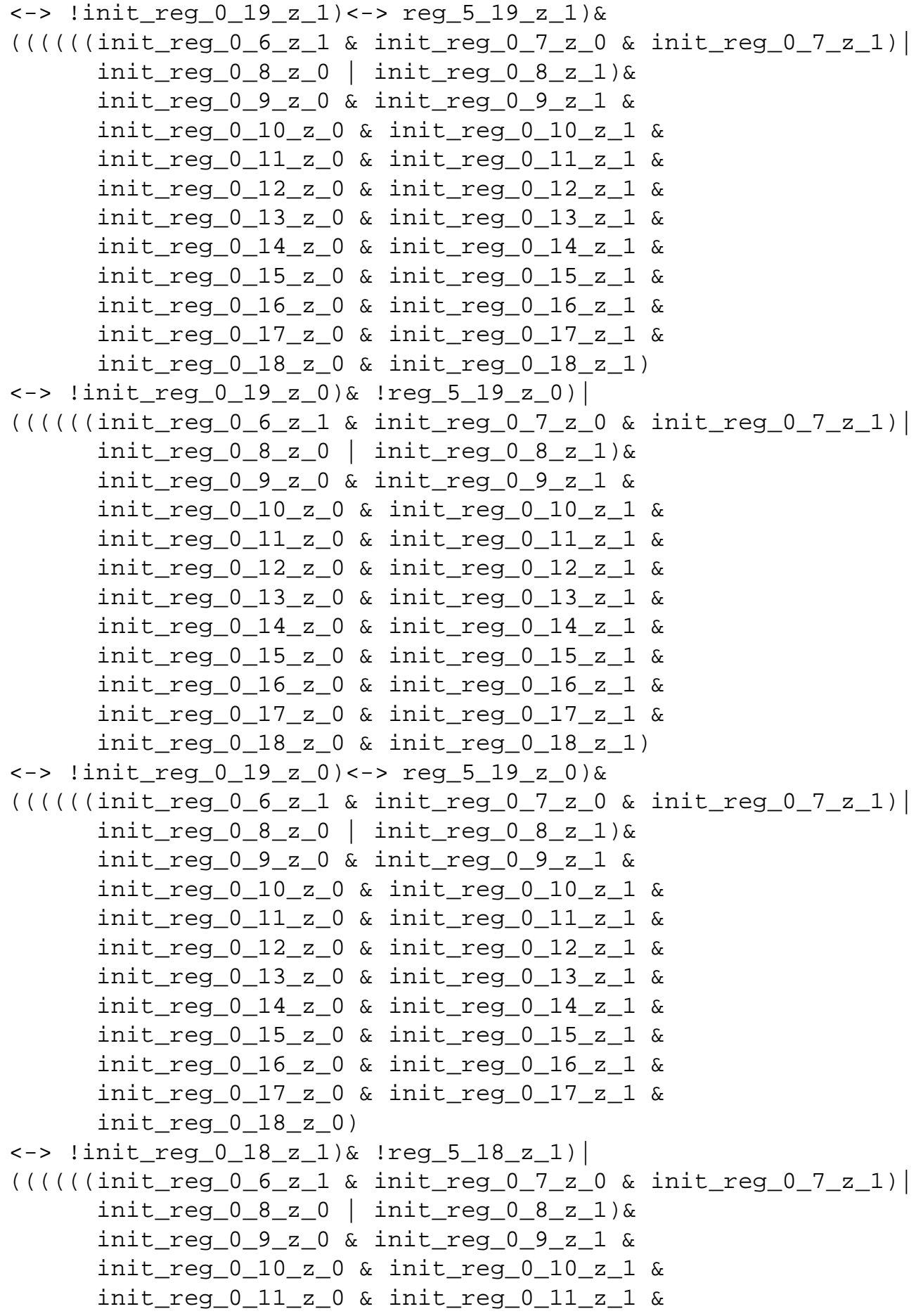




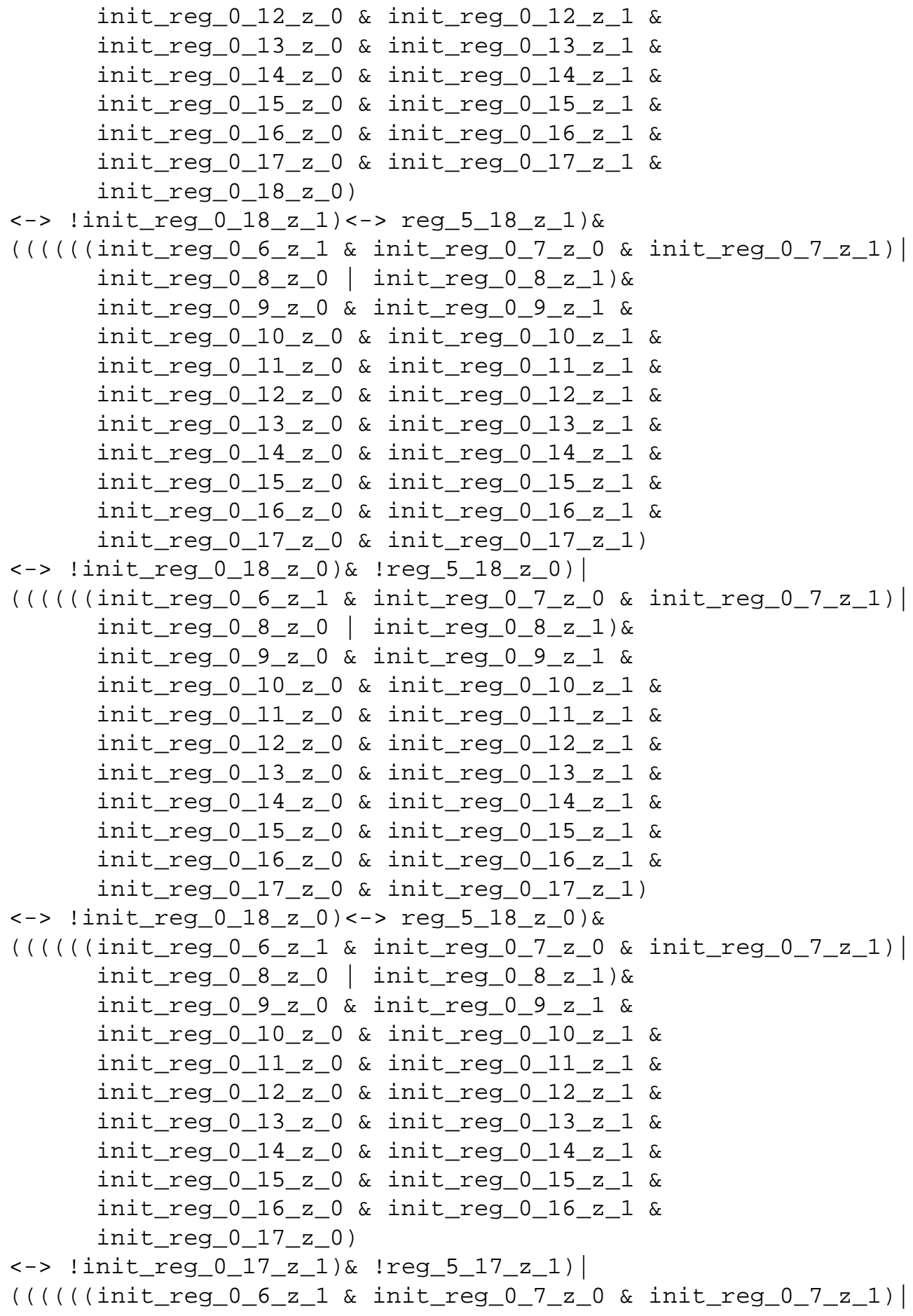




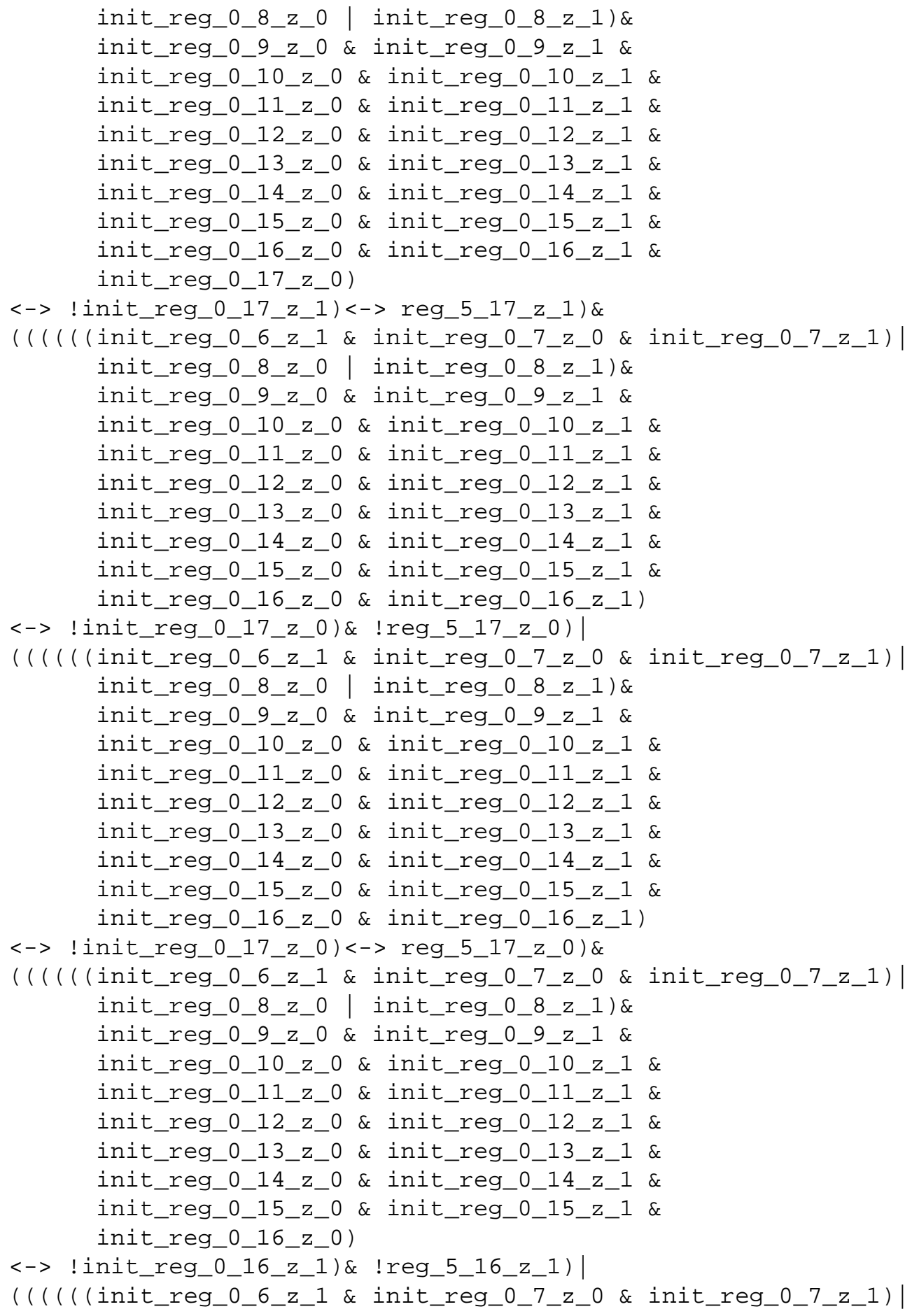




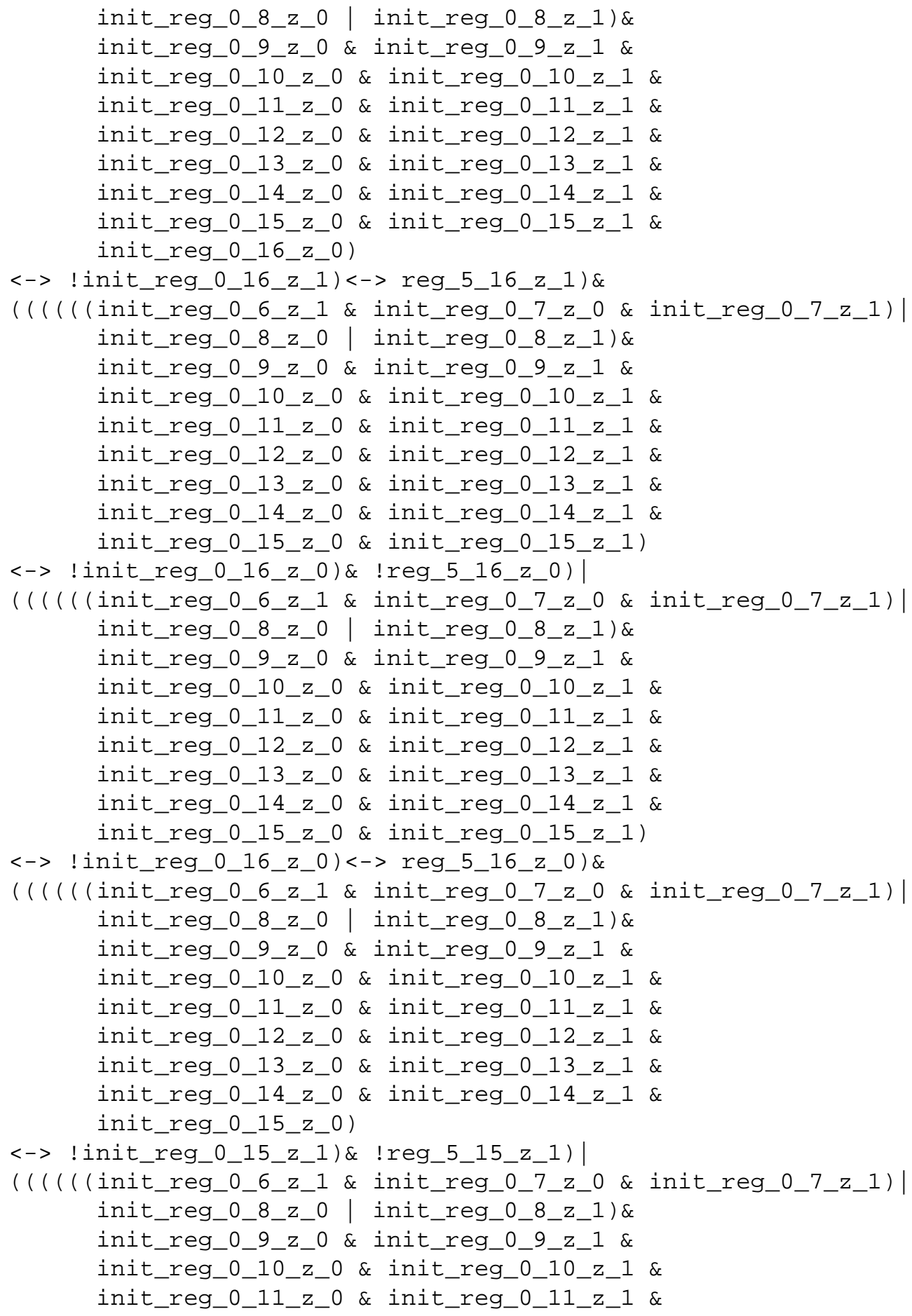




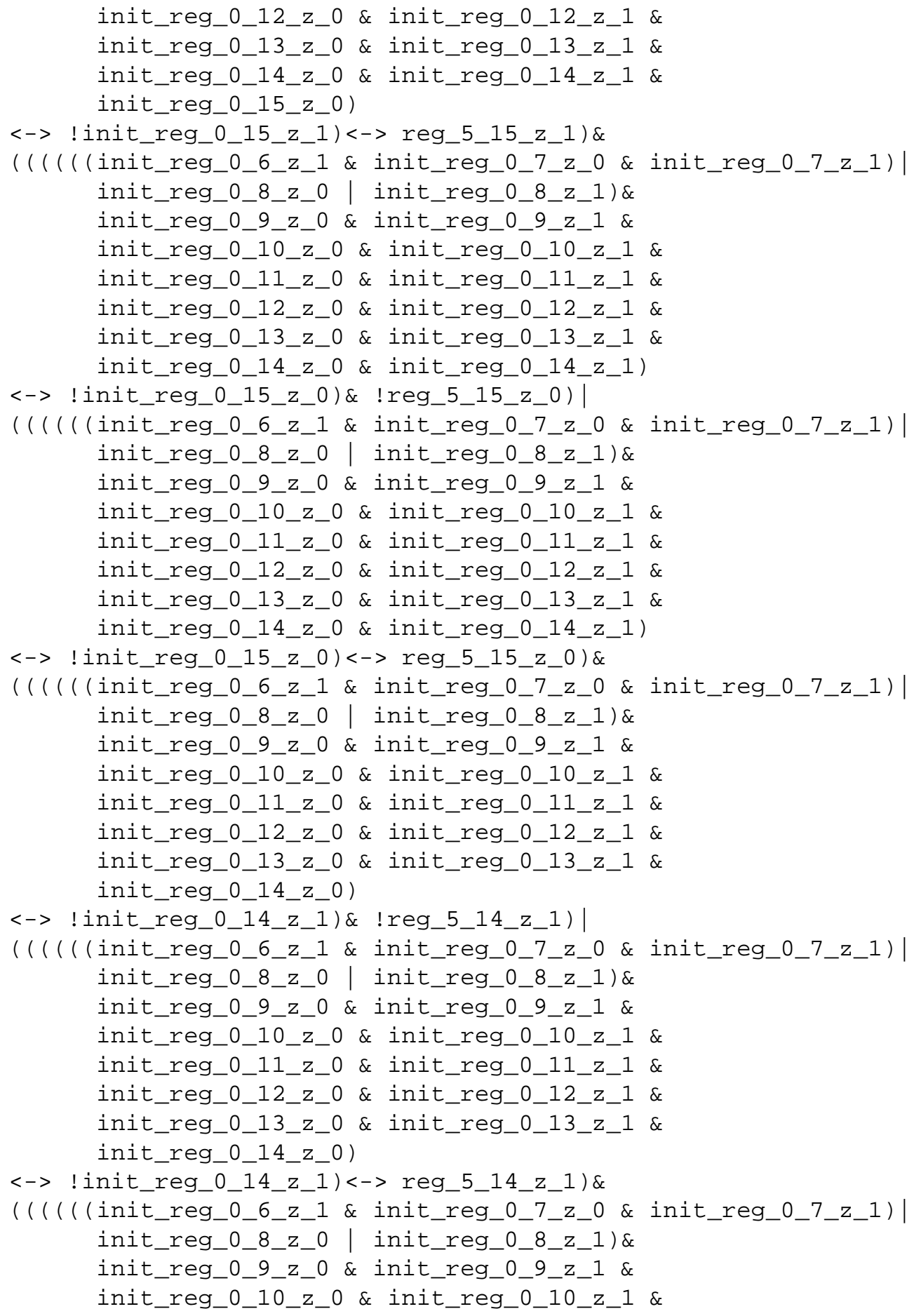




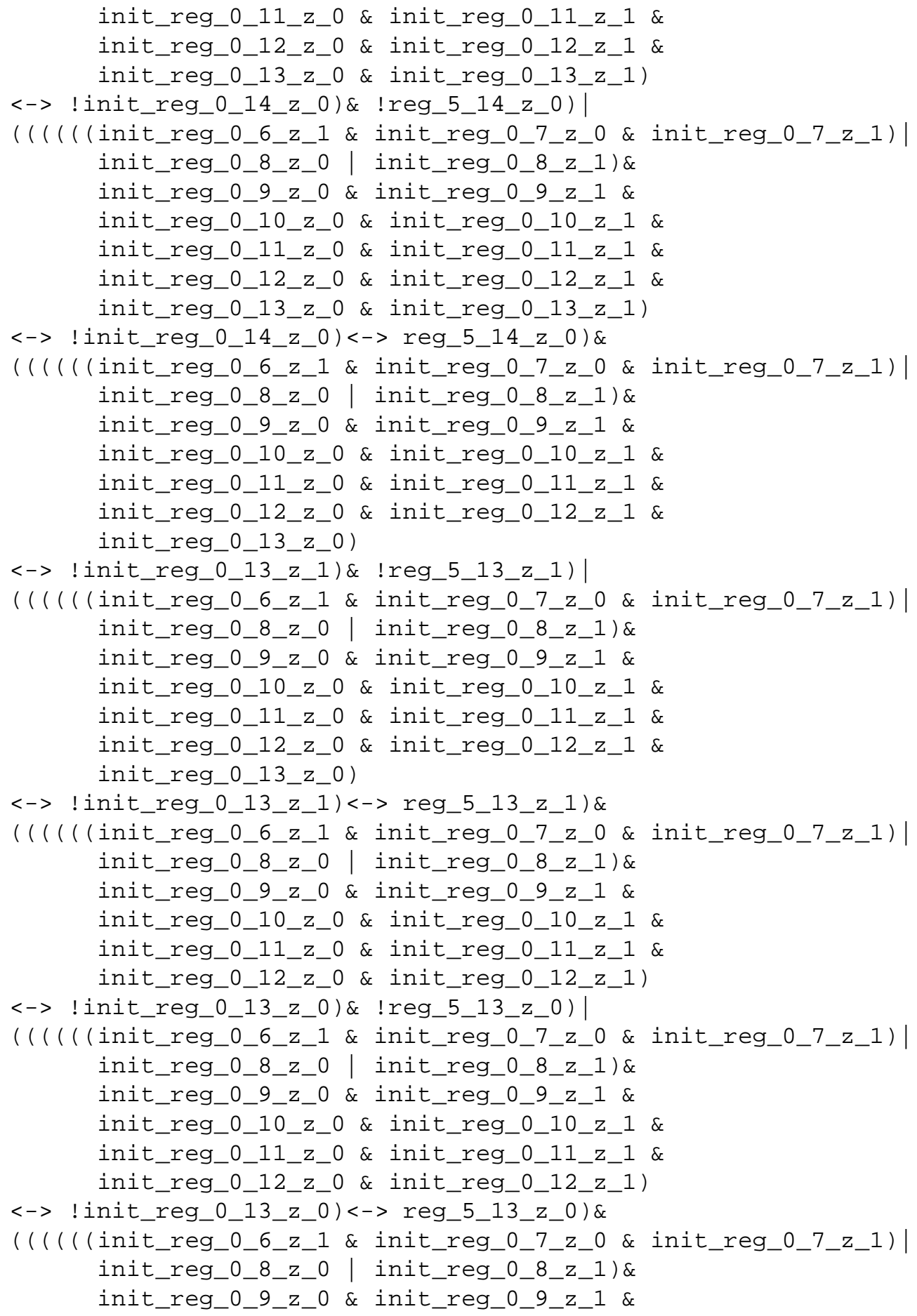




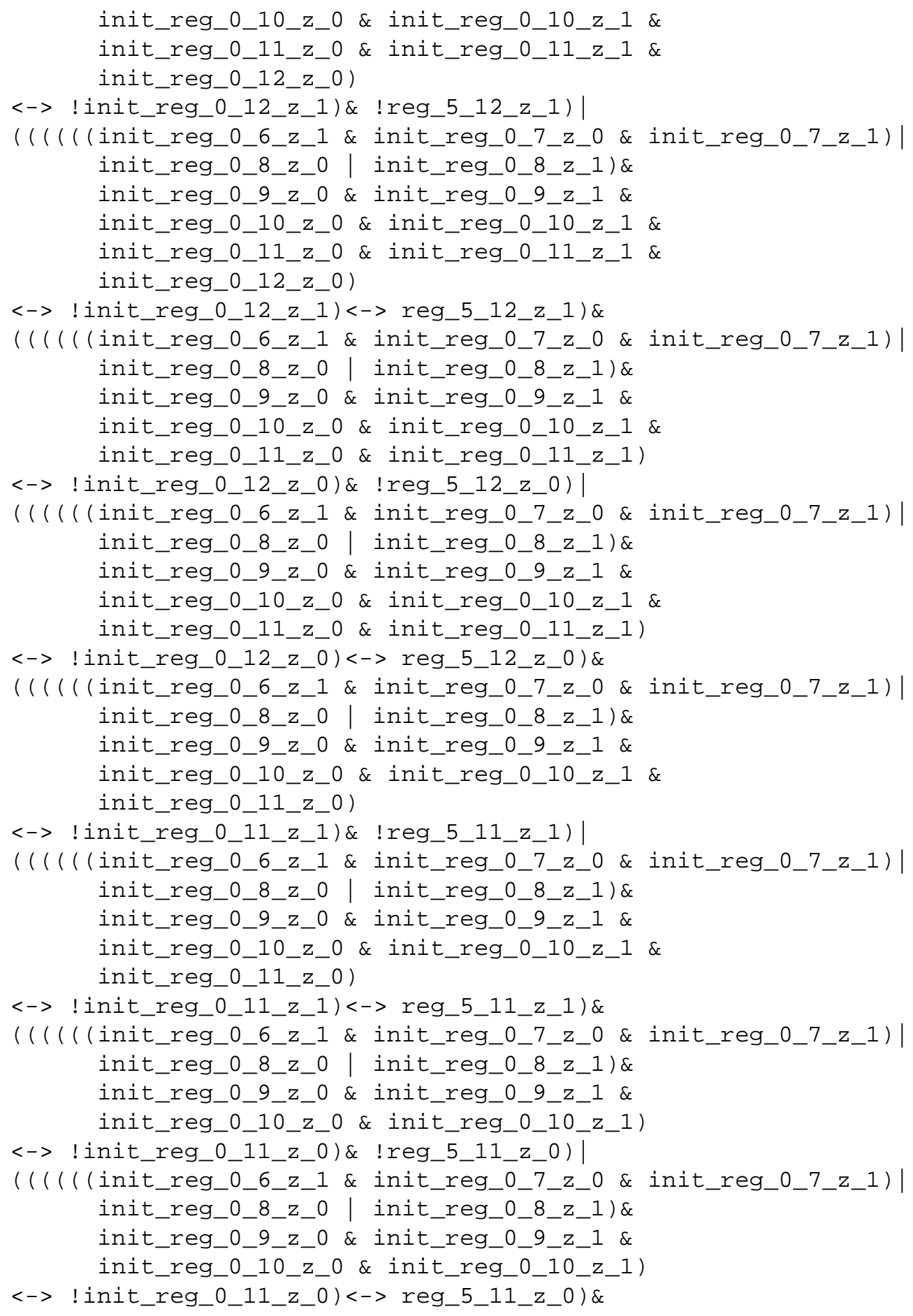




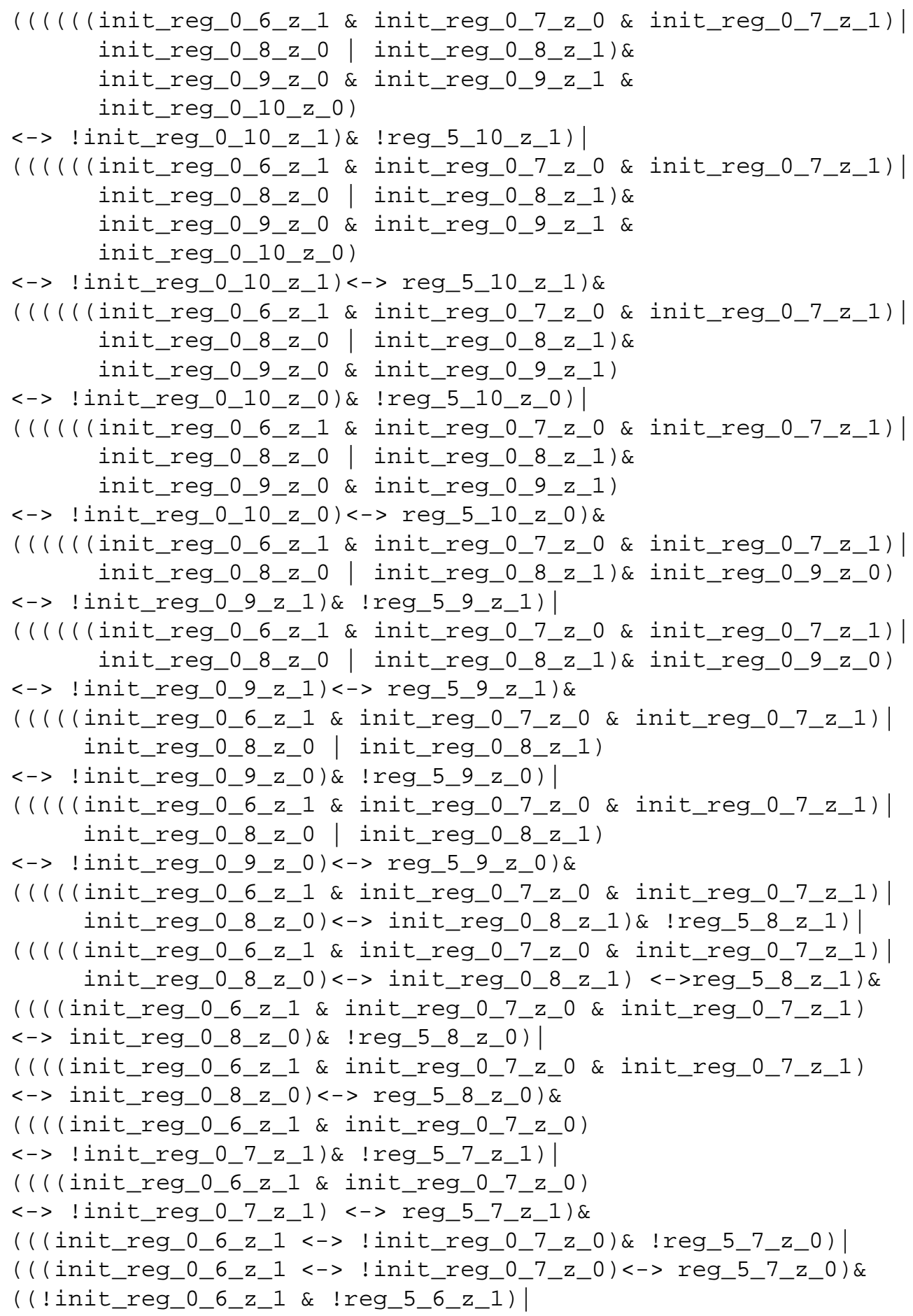




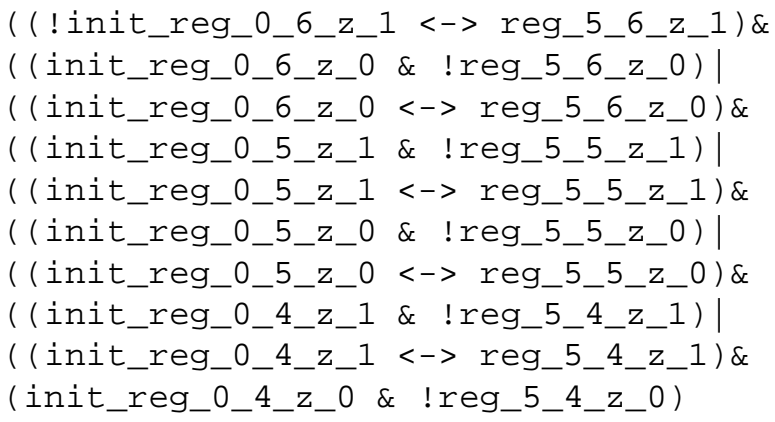

\section{B.4 HASH}

SPEC -- MC

-- the correct initialization of the control path implies correct -- memory synchronization

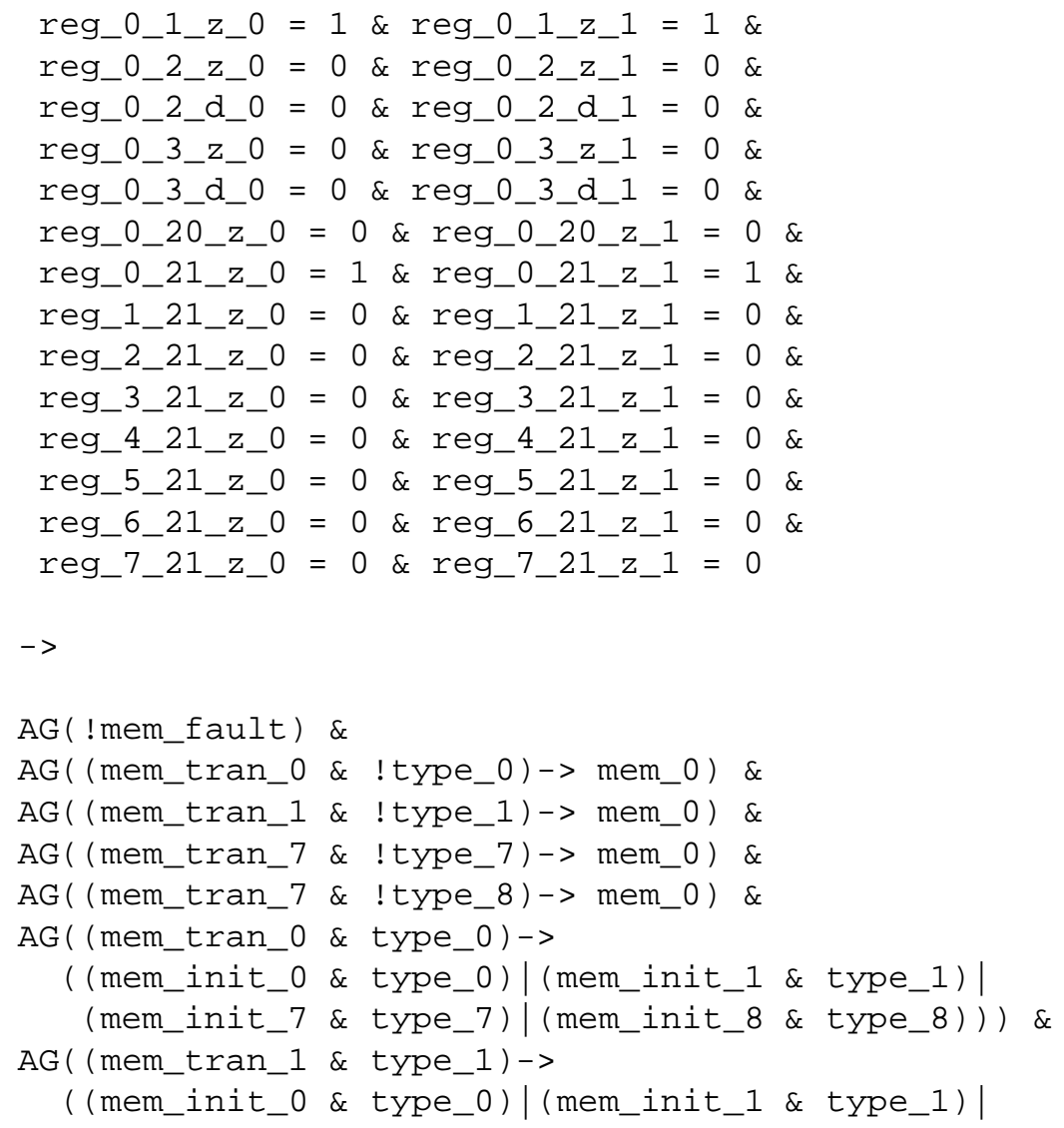




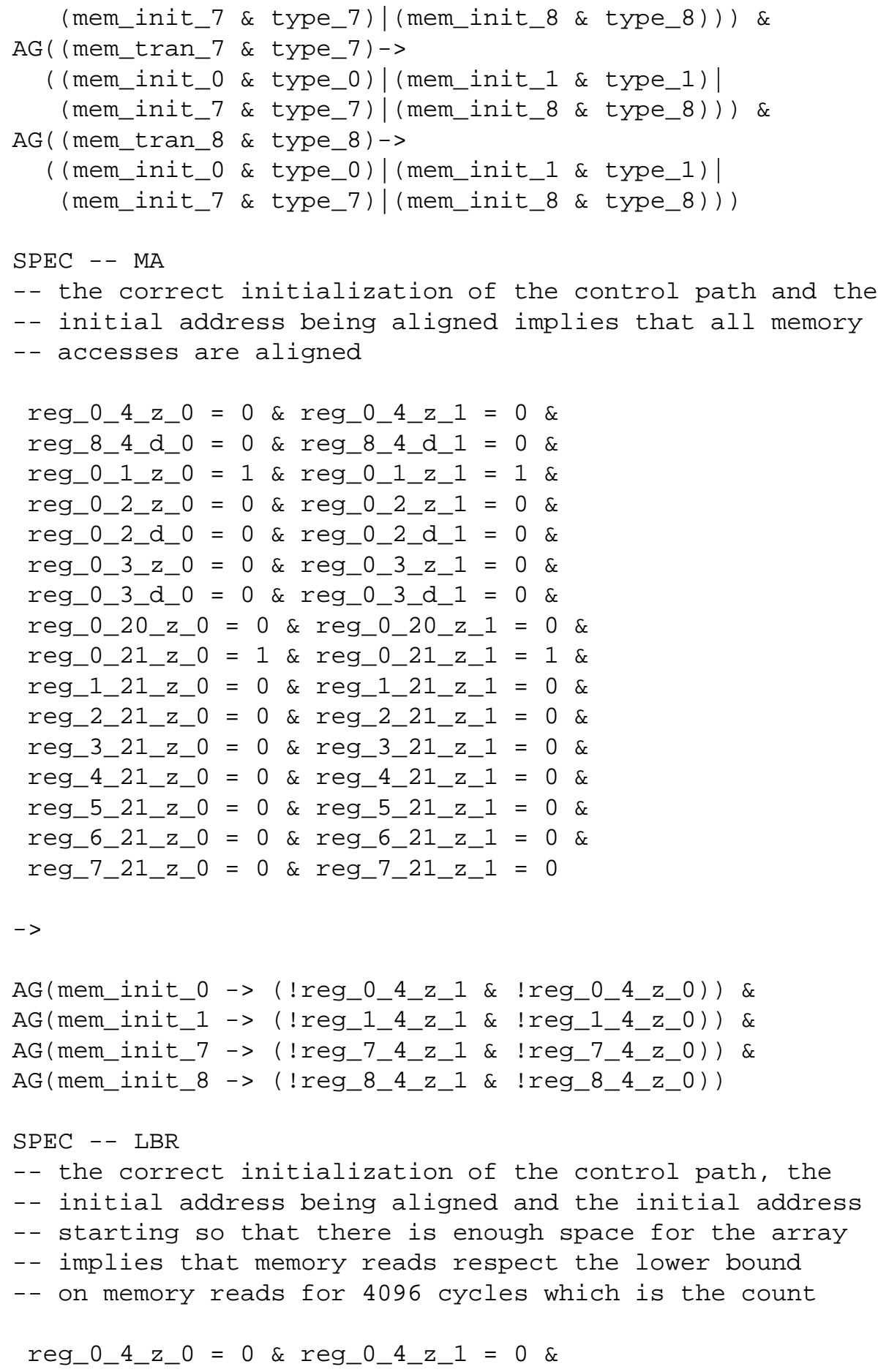




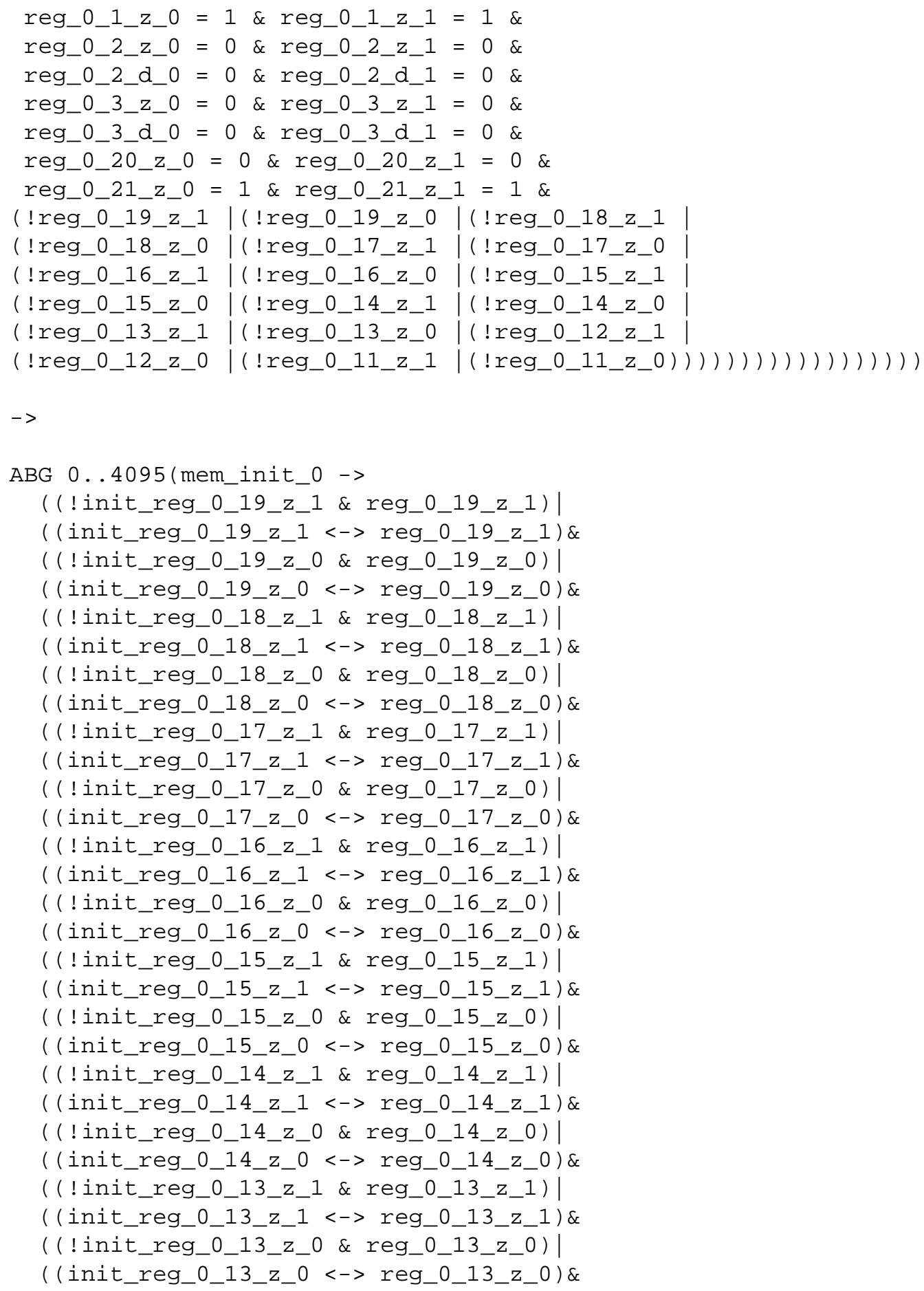




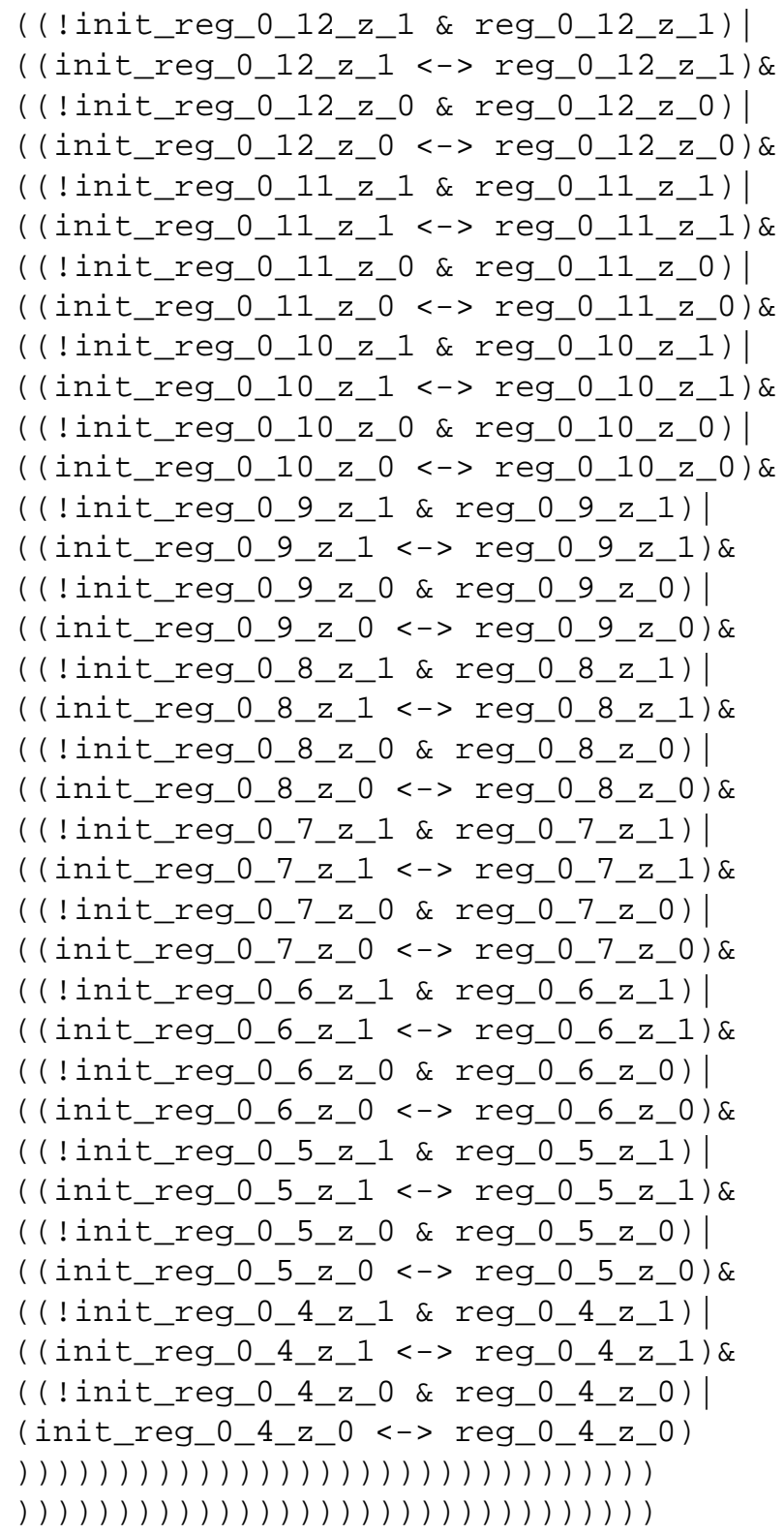




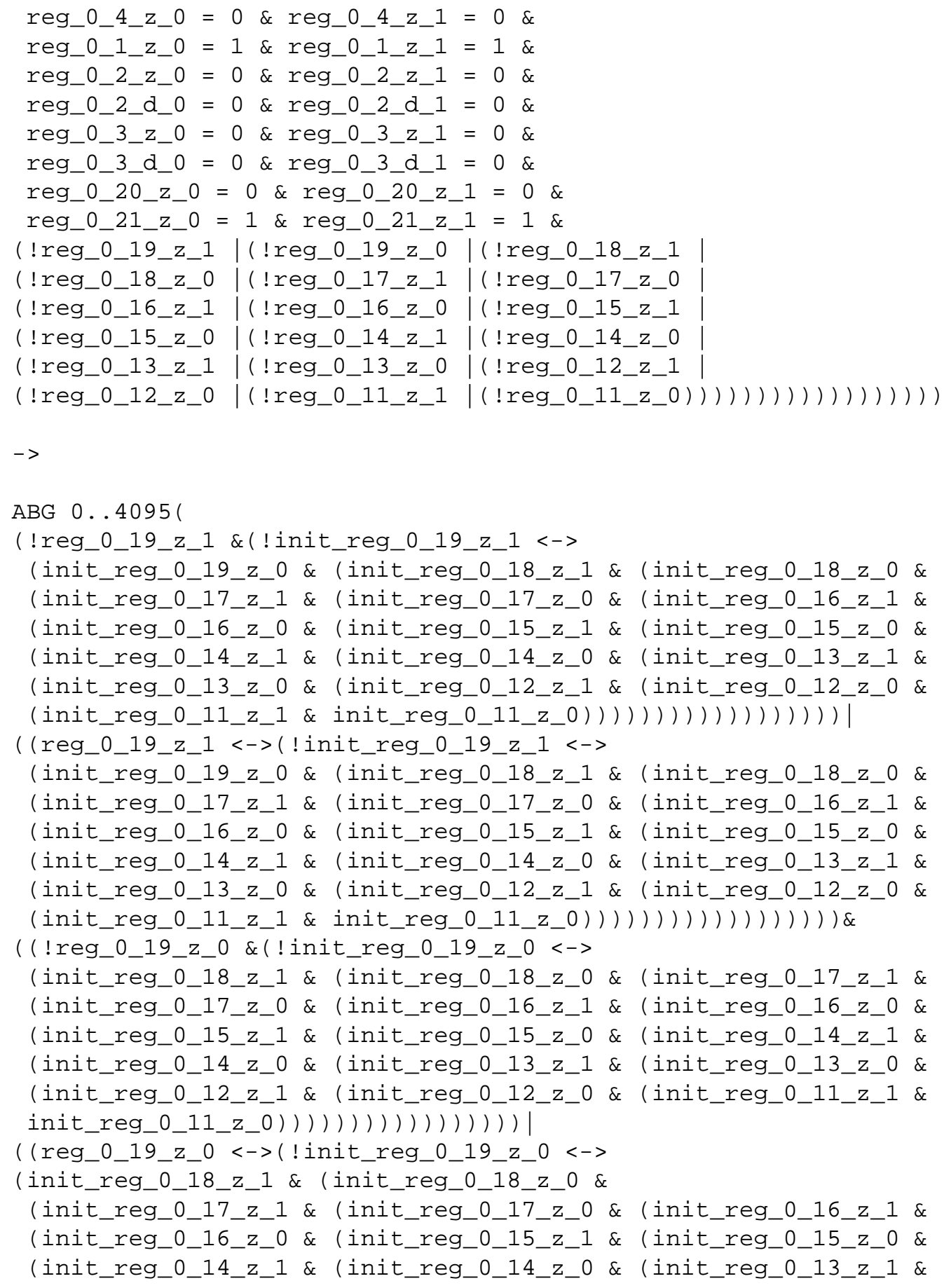




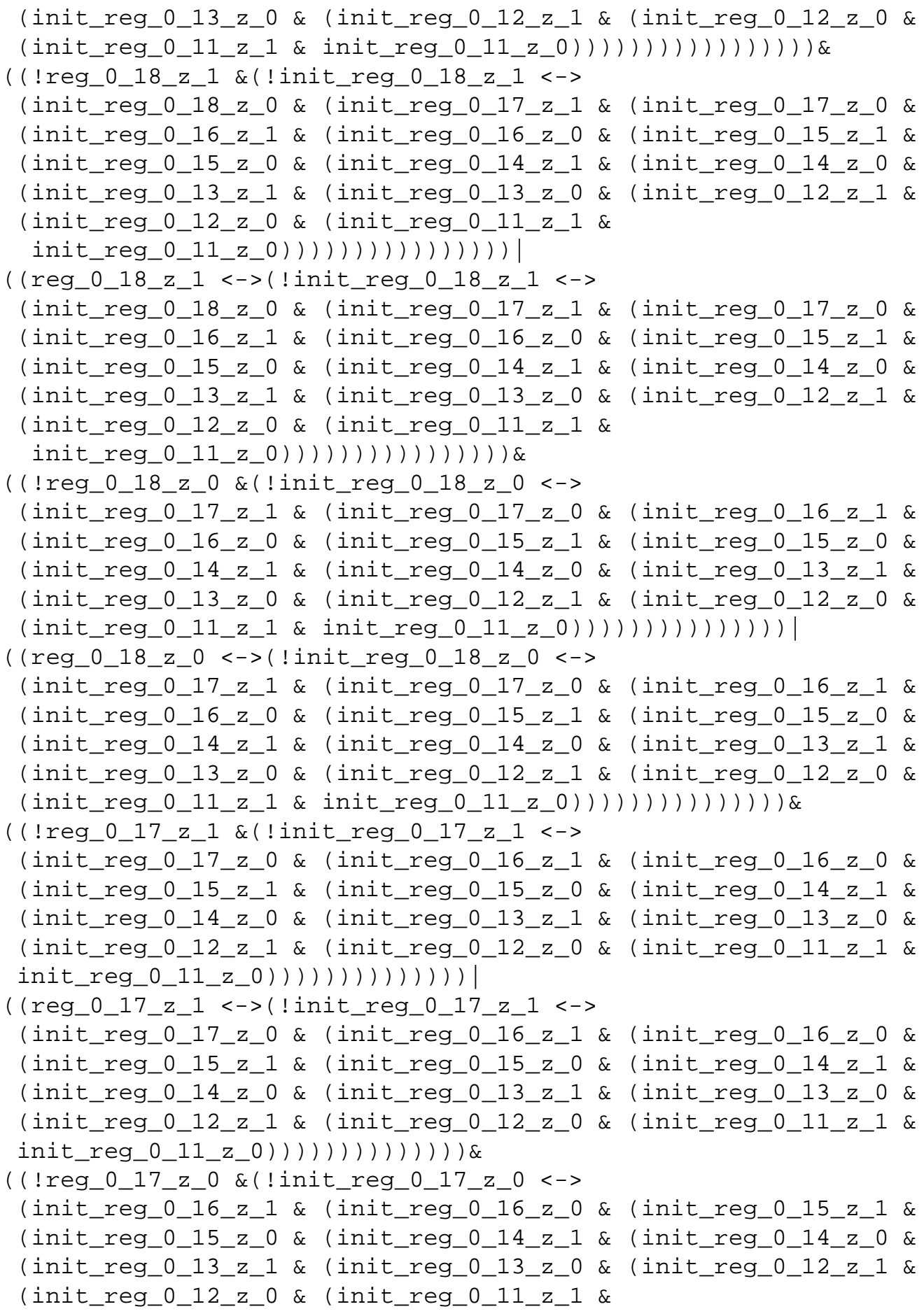




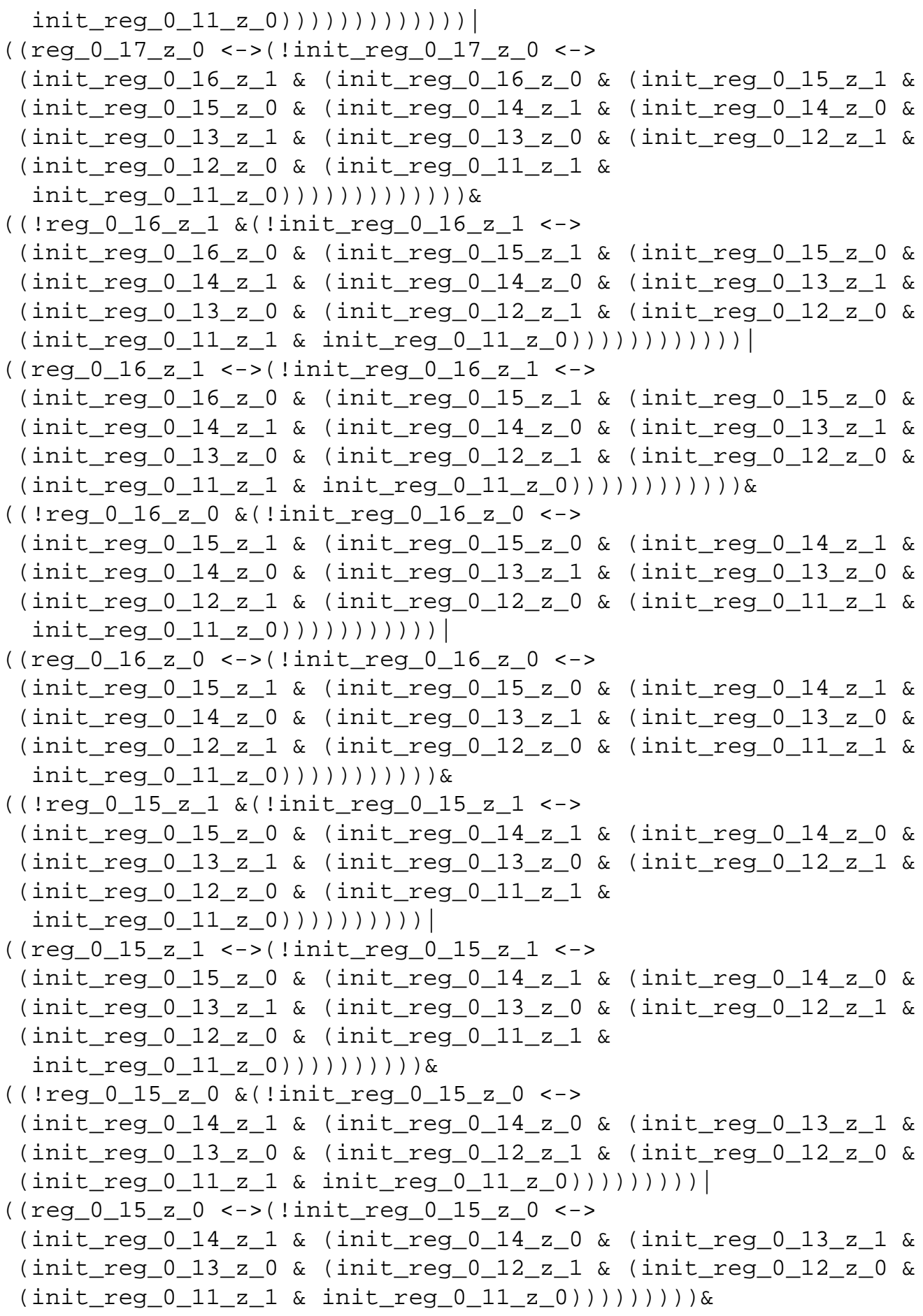




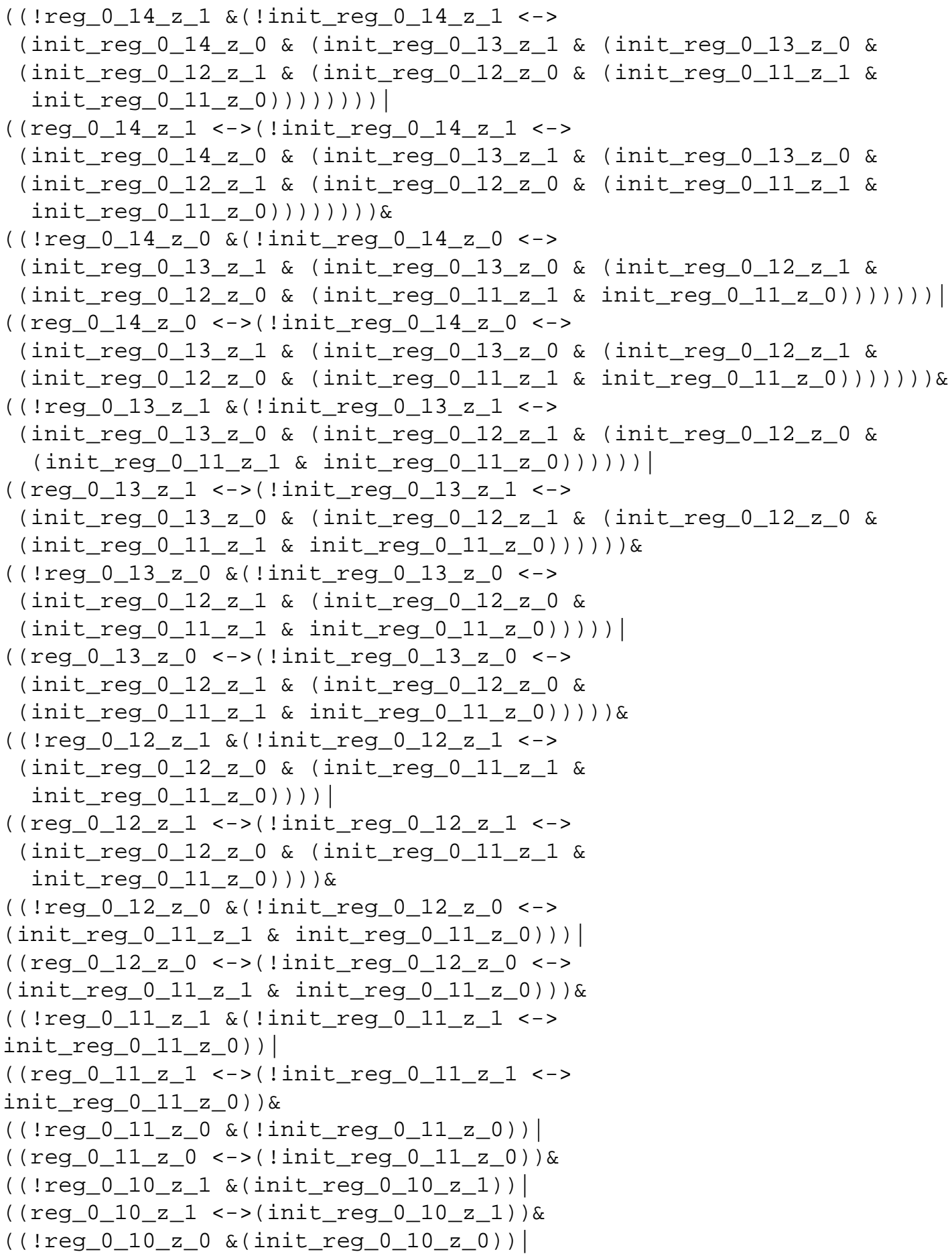




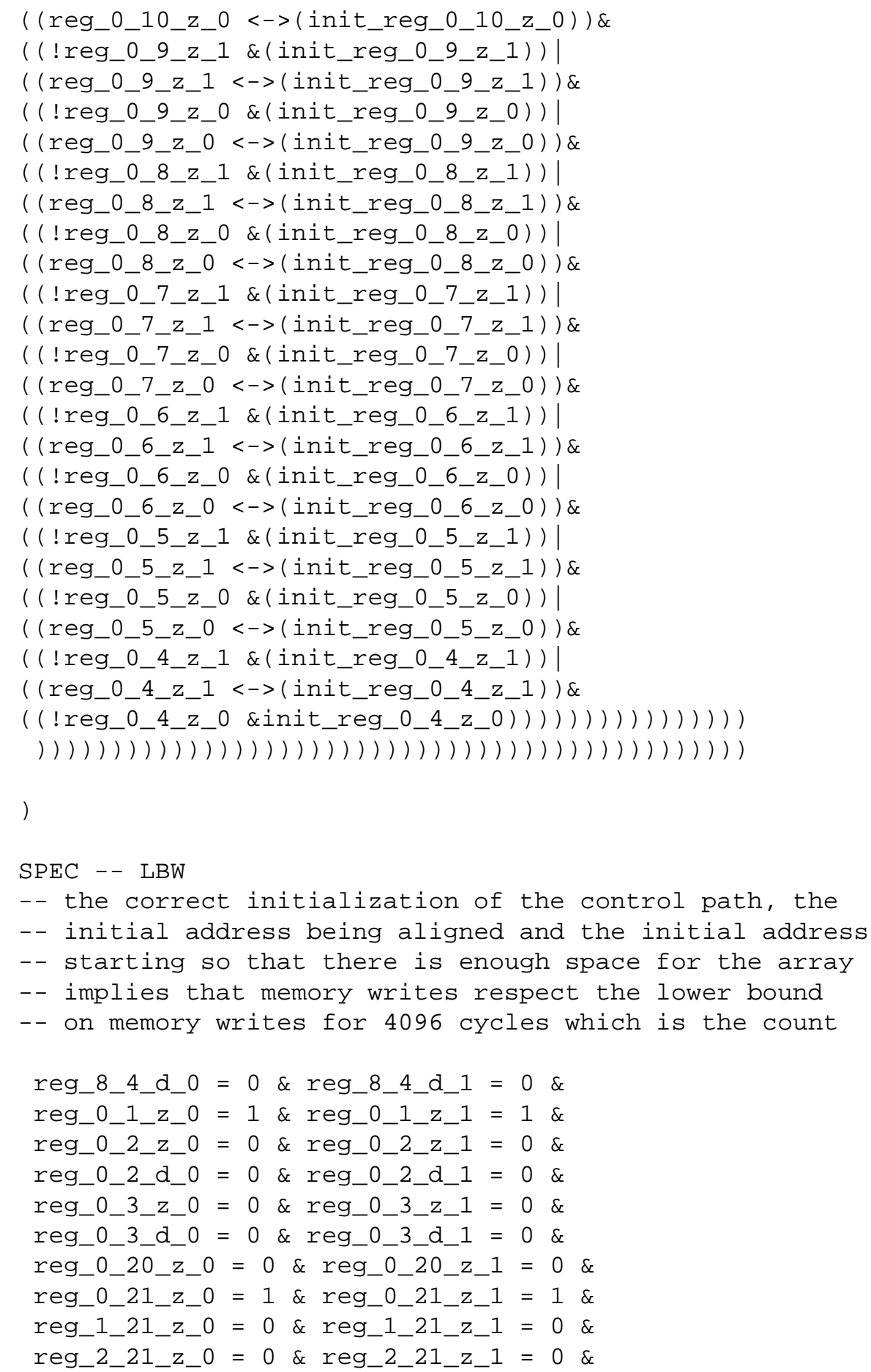




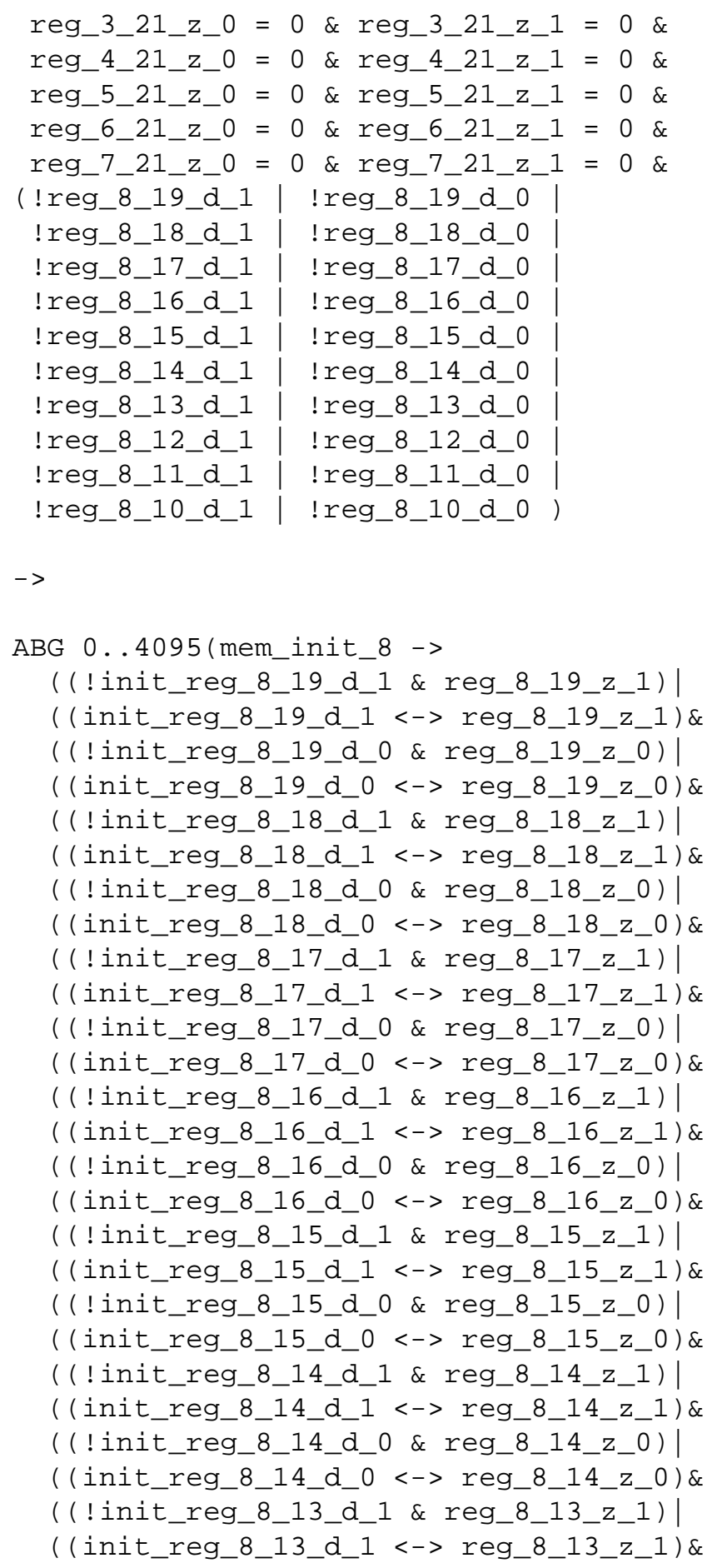




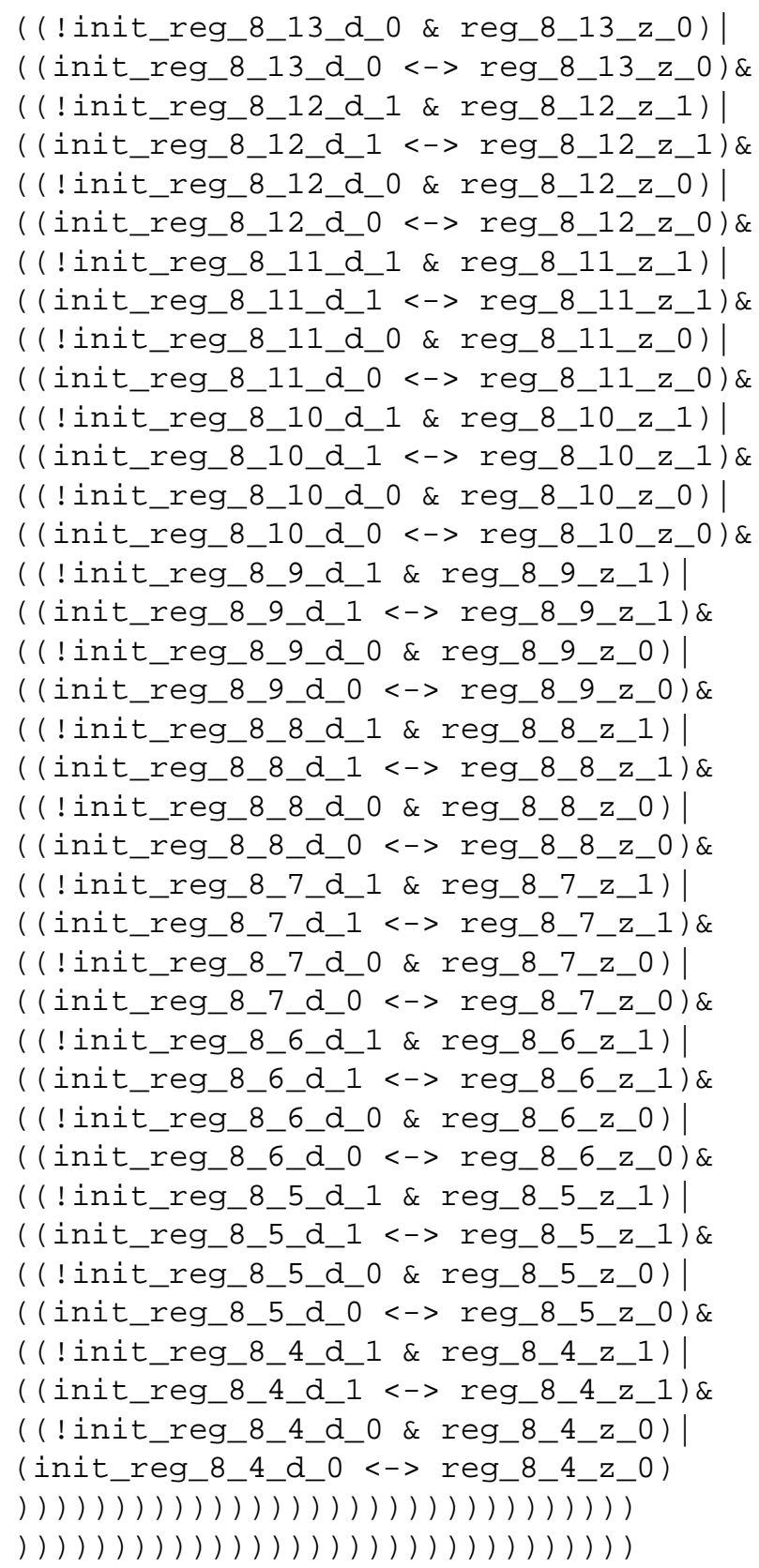

